\title{
Métodos de pontos interiores aplicados ao fluxo de carga ótimo utilizando coordenadas cartesianas ${ }^{\ddagger}$
}

\author{
Adriano Thomaz
}

Oricntador:

Prof. Dr. Aurelio Ribeiro Leite de Oliveira

Dissertação apresentada ao Instituto de Ciências Matemáticas e de Computação - ICMC-USP, como parte dos requisitos para obtenção do título de Mestre em Ciências de Computação e Matemática Computacional.

\section{USP - São Carlos}

Abril/2003

${ }^{\ddagger}$ Este trabalho contou com apoin financciro da FAPESP. 
A Comissão Julgadora:

Prof. Dr. Aurelio Ribeiro Leite de Oliveira

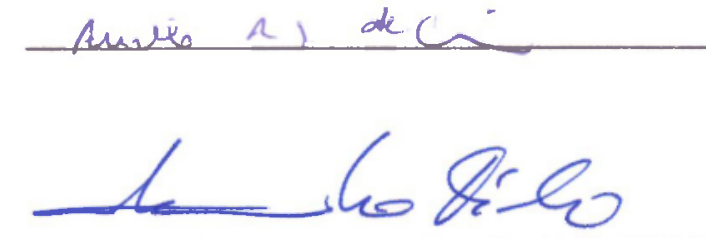

Prof. Dr. Marinho Gomes de Andrade Filho

Prof. Dr. Leonardo Nepomuceno

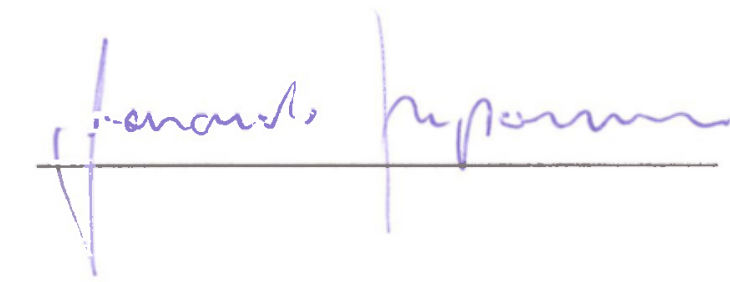


Para Irene (em memória) 


\section{Agradecimentos}

$\grave{\Lambda}$ Deus por estar sempre comigo e iluminar men caminho.

Ao men orientardor Aurelio Ribeiro Leite de Oliveira, pela amizade, pela confiança deposilada no desenvolvimento deste trabalho, por acreditar em mim e estar sempre disposto a me ajurlar com paciencia e declicação.

A meus pais Artete e Eduarde, pelo amor, carinho, fue sempre priorizaram minha educação e acreditaram em mim, me apoiando e auxiliando para que cu pudesse realizar neus trabalhos e sonhos.

Aos professores Geraldo Roberto Martins da Costat, Secuudino Soares filho, e especiahmente para Selma 1I. Vasconcelos Arenales, Marcos Nereu Arenales o Artur 1)arezo Filho.

A formandia pelo apoio, amizade, anor c comprecusaio.

A Marrilia, Cesília, bedson, e de todes da família pelo tolal apoio e compreensăo.

Aos amigos Tatiana, Stélio, Michelle, Priscilla e Renata pelo incentivo. Los anigos do Laboratório de Otimizaçio do ICMC-USP, Silvio, Ricardo, Fabiano, folder, Carla, Gecirlei, Fabrizio, Lilian, em especial para Andró, Cecília, Glancia o Kolly.

Aos funcionários do ICMC-USP que, direta on indiretamente, contribuíram crom este traballio.

$\grave{\Lambda}$ todos os meus amigos de São Carlos.

A FAPRSP pola credibilidarle e apoio financeiro.

E finalmente a todos cue colaboraram indiretamente na realização deste trabalho. 


\section{Resumo}

Os mét odos de pontos interiores primal-dual e preditor-corretor sio desenvolvidos para o

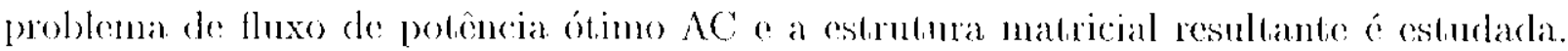
Foi adolada a representação do problema através de coordenadas cartesianas das tensões una vez que neste modelo a Hessiana do problema ó constante e a expansão em Taylor é exata para o termo de ordem dois. Além disso, o cálento do termo de correção do método preditor-corretor pode ser leito de forma menos custosa computacionalmente. Por outro lado, a vantagem em se trabalhar com coordenarlas polares, que modelam mais facilmente os limites de magnitude de tensão, perde importancia devido ao tratamento Ie desigualdades eficiente proporcionado pelos mótorlos de pontos interiores, permitindo uma revisâo dos procerdimentos geralmente adotades. Assim, a utilizaçiò de coordenadas cartesianas surge como una abordagem natural, pois apresenta uma formulaçaio mais simples que as coordenatas polares. A aplicacäo do método de Newton is condiçōes de otimalidade leva a um métorle de pontos interiores primal-rlual específico para cste

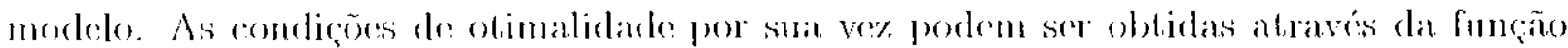
lagrangiana do problema onde as restriçoes de desigualdade säo representadas por funçóces

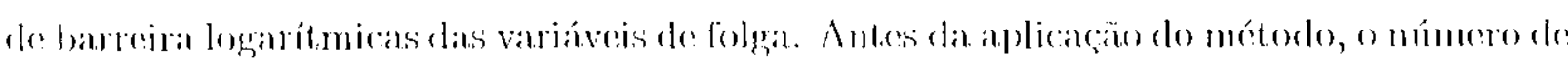
variáveis do problema é reduzido atravós da climinação de variáveis duais livese cue serão calculadas no final. Lista redução nấo altera a estrutura esparsa do problema. O sistema linear resultante pode então ser reduzido a duas veyes a cuantidade do número de barras da rede de transmissão. Além disso, a matriz resultante é simćtrica em estrutura. Esta característica pode ser explorada de forma cliciente reduzindo o esforço compulacional por iteraçân. 


\begin{abstract}
The primal-dual interior point methods are developed to the AC optimal power flow and the resulting matricial structure is studied. The representation of the tensions through cartesian coordinates is adopted, once that Fessian of the problen is constint and the expansion in Taylor is aceurate for the second order term. The advantage of working with polar coordinates, that easily motel the tension magnitudes, lose importance due to the efficient treatment, of incerualities moportionated by the interior point methods. These methods are developed applying Newton's methods to the optimality conditions of the problem. Before the application of the method, the number of variables of the problem is reduced through the elimination of free dual varialbles. This reduction does not modify the sparse pattern of the problem. The linear system obtained can be redued to the dimension of twice the number of buses. Moreover, such matrix is symmetric in strueture. This feature can be explored reducing the computational effort per iteration.
\end{abstract}




\section{Conteúdo}

$\begin{array}{ll}\text { Introdução } & 1\end{array}$

1 Métodos de Pontos Interiores 3

1.1 Aspectos Gerais . . . . . . . . . . . . . . . . . 3

1.2 Problemas lincares . . . . . . . . . . . . . . . . 4

1.3 Método de Newton para Uma Variável . . . . . . . . . . . . 5

1.1 Método de Newton para Várias Variáveis . . . . . . . . . . 5

1.5 Mćtodo Primal-Dual Afin Escala. . . . . . . . . . . . . . 6

1.6 Método Preditor-Corretor . . . . . . . . . . . . . . . . . . 9

1.7 Cálculo das Direçõos nos Mótodos de Pontos Interiores . . . . . . . . 11

2 Fluxo de Carga $\quad 13$

2.1 Aspectos Gerais. . . . . . . . . . . . . . . . . . . 13

2.2 lommulacio do Prohlemal . . . . . . . . . . . . . . . . . . 14

2.3 Modelingem . . . . . . . . . . . . . . . . . 15

2.3 .1 Linhats de Transmissão . . . . . . . . . . . . . . . . 15

2.3 .2 Fluxos de Potencia Ativa o Realiva . . . . . . . . . 16

2.4 Formulação Matricial . . . . . . . . . . . . . . . . 17

3 Problema de Fluxo de Carga Ólimo AC 21

3.1 Motivacăo . . . . . . . . . . . . . . . . . . . . 21

3.2 Formulacäo do Problema . . . . . . . . . . . . . . . 22

3.2 .1 Prohlema Relaxarlo . . . . . . . . . . . . . 23

3.3 Simplificaca da Matriz IIessiana . . . . . . . . . . . . . 24 
4.1 Metoclologia . . . . . . . . . . . . . . . . . 25

4.2 Função Barreira Logarítmica . . . . . . . . . . . . . . . . . . 26

4.3 A Função Lagrangiana . . . . . . . . . . . . . . . . . . . 27

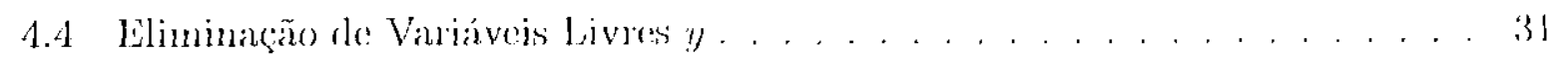

4.5 Mćtodo de Pontos Interiores Primal-Dual . . . . . . . . . . . . . 32

4.5 .1 Diregoes de Newton . . . . . . . . . . . . . . . . . 34

4.6 Método Preditor-Corretor . . . . . . . . . . . . 35

4.7 Detalhes de Implementação $\ldots \ldots \ldots \ldots$

4.7 .1 Consideraçoes Iniciais. . . . . . . . . . . . . . . . 365

4.7 .2 Atualização das Variáveis . . . . . . . . . . . . . 36

4.7 .3 Cálculo do Comprimento do Passo f . . . . . . . . . . 36

4.7.1 Redução do Parâmetro de Barreira . . . . . . . . . . . 37

4.7 .5 Critério de Convergêtucia . . . . . . . . . . . 37

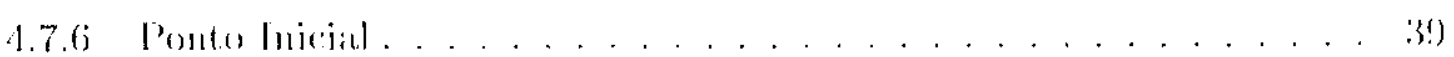

5 Resolução do Sistcma Linear

5.1 Estrutura Matricial . . . . . . . . . . . . . . . . 45

6 Resultados Computacionais $\quad 47$

6.1 Implementaçăo e Ambiente . . . . . . . . . . . . 17

6.2 Sistemas . . . . . . . . . . . . . . . . . . 48

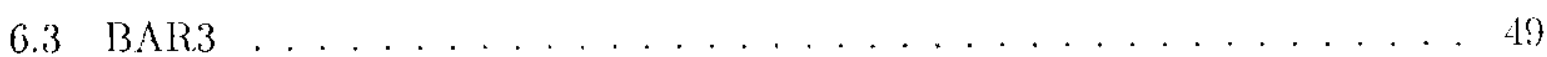

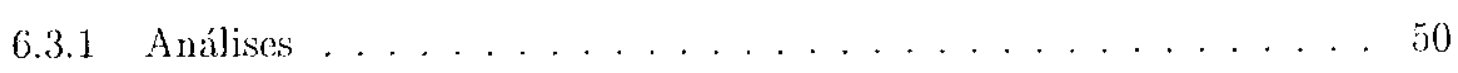

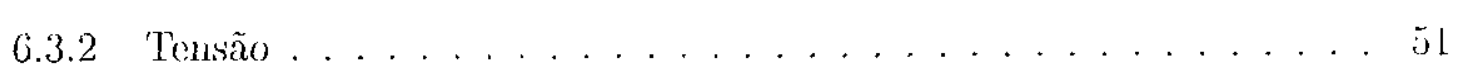

6.3 .3 Potencia Ativar . . . . . . . . . . . . . . 51

6.3.4 Normas Residuais Relativas . . . . . . . . . . . . . . . 53

6.3.5 Fluxo e Perda de Potência Ativa nas Linthas de Transmissão . . . . 54

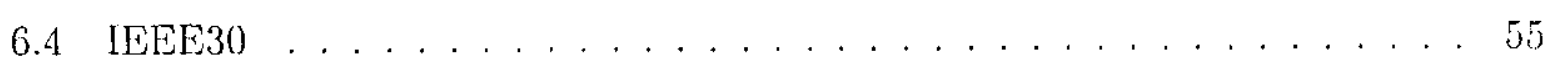

6.4 .1 Análises ..................... 55

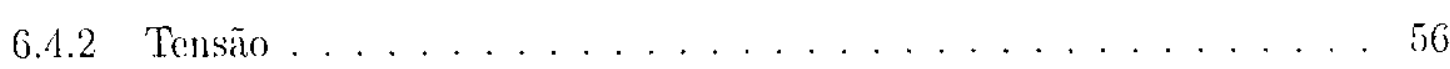


6.4 .3 Potencia $\Lambda$ tival $\ldots \ldots \ldots \ldots \ldots \ldots \ldots$

6.4 Normas Residuais Relativas . . . . . . . . . . . . . 58

6.4.5 Fluxo e Perda de Potência Ativa nas Linhas de Transmissão . . . 59

7 Conclusões

8 Propostas Futuras

Bibliografia

Apêndice

Relatórios de Saíla da Implementação 


\section{Lista de Figuras}

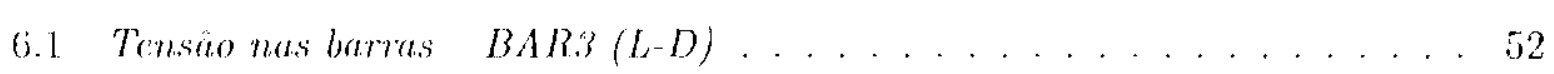

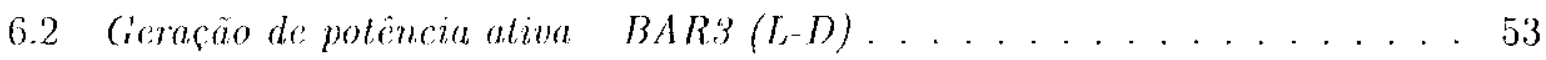

0.3 Normas residuats relativas - BAR3 (L-D) . . . . . . . . . . . . 53

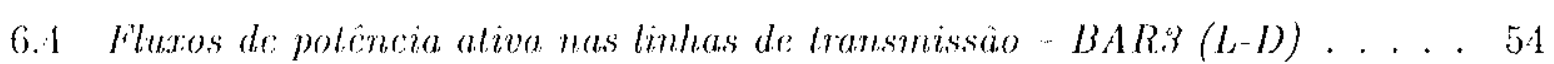

6.5 Tensão nas barras - IELE, $(L-D)$. . . . . . . . . . . . . . . . . . 56

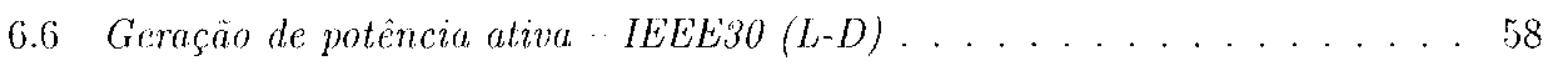

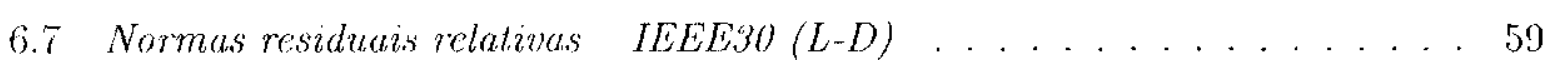

(j.8 Fluros de potência aliva nas linhas de transmissão - MELS30 (L-D) . . . . 59

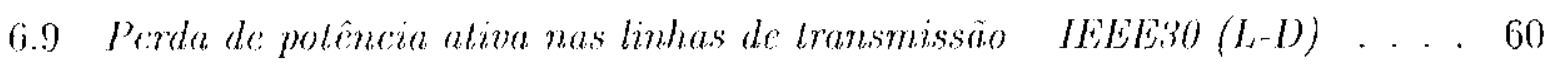

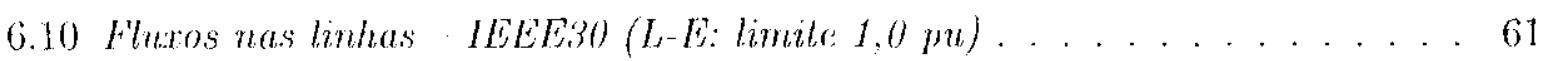

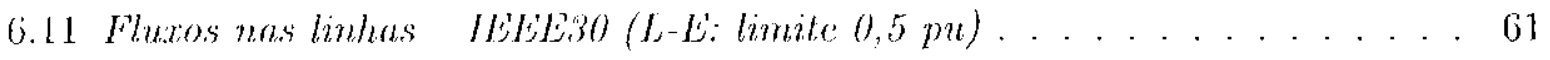

6.12 Perda nas linhas de transmissio - IEEE30 (L-E: limile 1,0 pu) . . . . . 64

6.13 Perda nas linhas de transmissäo JEEESO (L-E: limite 0,5 pu) . . . . . 64 


\section{Lista de Tabelas}

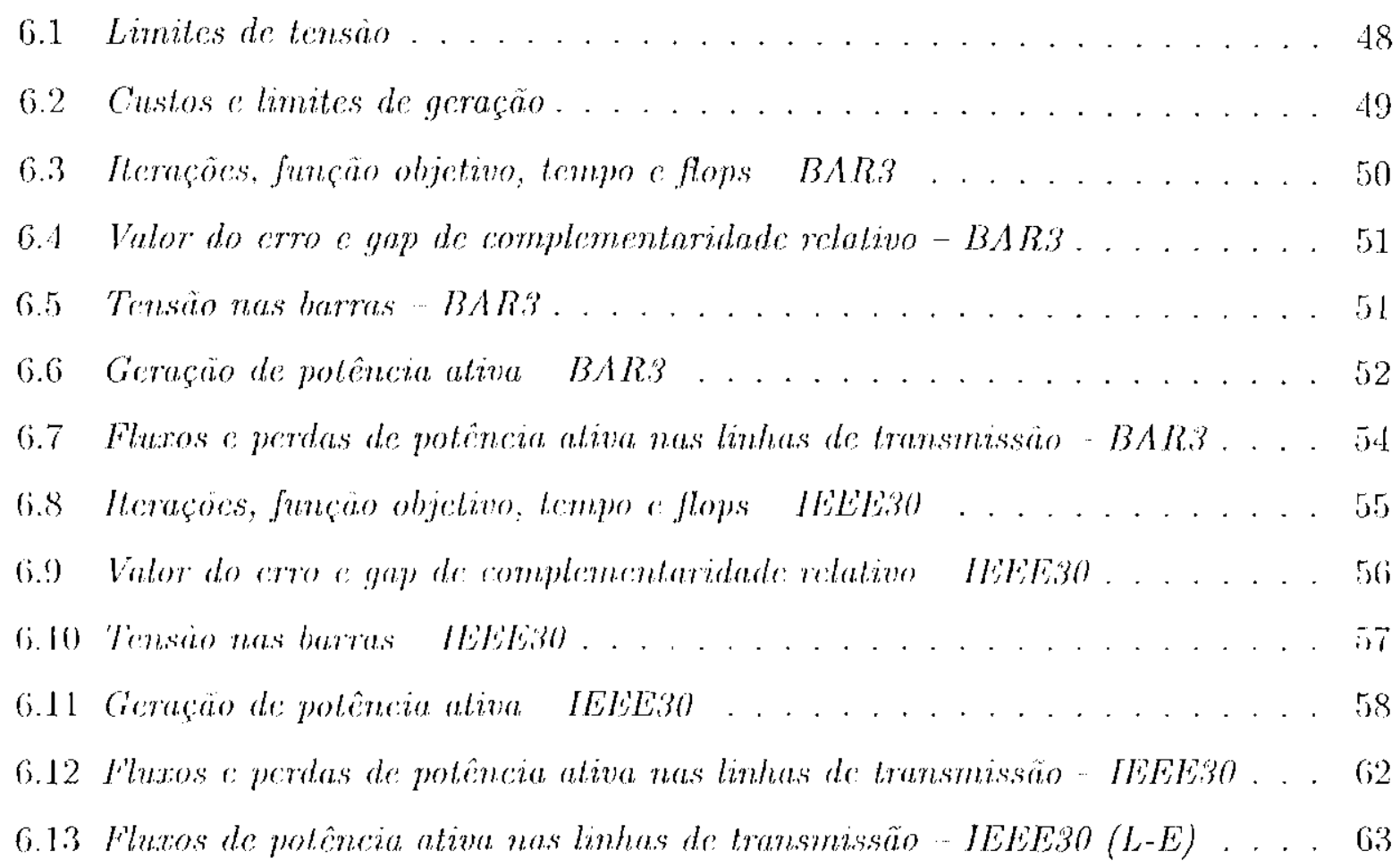




\section{Introdução}

O problema de fluxo de potencia ótimo (ou fluxo de carga ótimo) ó unn problema de programação näo-linear de grande porte não convexo. Torna-se complicado na prática pela presença de am grande número de variáveis discretas. Data sua importâneia no plancjauneuto e operação de sistemas de potêtecia os problemas de fluxo de potência ótimo têm sido assunto de intensa pesquisa.

As redes clétricas estão atualmente operando muito carregadas, assim as ferranentas de planejamento e opexaça precisam trabalhar com problemas com alto grau de nâo-linearidade no comportamento do sistema.

Resolver problenas de fluxo de potencia ótimo eficientemente de mancira näolinear é un tópico muto complexo. Uma técnica mais recentemente ntilizada para a resolução de problemas de fluxo de potencia ótjmo de grankle porte $\Lambda \mathrm{C}$ é a dos mótodos de pontos interiores $[14,20]$. Uma estrutura de dados bem elaborada e o uso eficiente de técnicas de esparsidade toman estes métodos muito atrativos.

Cina característica comum desses trabalhos ó que problomas do programação när-incar tém sido resolvidos eficientemente pelos métodos de pontos interiores para programaçăo näo-linear derivados da abordagem de função barreira logarítmica. Esssa abordagen foi introduzida por Frisch [5], e desenvolvida como uma ferramenta para programação não-linear por Fiacco e McCormick [4]. Embora tenha sido desenvolvida para resolver problemas de programação năo-linear em geral, foi no campo da programaçio

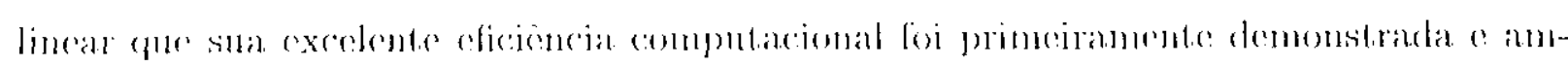
plamenter anceita $[1,10,13]$. 


\section{Capítulo 1}

\section{Métodos de Pontos Interiores}

\subsection{Aspectos Gerais}

Descle 1985, prescuisas sobre métodos de pontos interiores passaram por imponente expansào, ambos en teoria e prática computacional. Dexivaçoes de métodos de pontos interiores estão semdo estendidas para resolver torlas as classes de problema de otimização, descle lincar até não-lincar e de convexos até näo-convexos, sendo o últiuno sem garantia con rolaçöo a sua convergencia. Da mesma forma, os métodos de pontos interiores estão também sendo aplicalos para resolver todos os lipos de problemas práticos. Sistemas de potencia ó uma dessas áreas onde cles estào sendo aplicados extensivanente. Deviclo ao tumanho e caracterislicas especiais desses problemas, os métorlos de pontos interiores tent-se mostrado uma alternativa viável especialmente para os problenas com alto gran de não-lincaridade $[8,23,27]$.

Vamos apresentar inicialnonte os metodos de pontos interiores para programaço linear, as deduçós desses métodos para problemas de fluxo de potencia ótima năo-lineares seräo mostradas mais adiante. 


\subsection{Problemas Lineares}

Neste capítulo vamos adotar a seguinte forma, denominada padraio, para o problema de programagäo linear:

$$
\begin{array}{rc}
\text { minimizar } & c^{t} x \\
\text { sujeito a } & A x=b \\
& x \geq 0,
\end{array}
$$

onde, $A \in \Re^{m \times n}$ com posto $m$ e os vetores columa $x, c$ e $b$ tem a dinnensão apropriadla. $O$ dual desse problema é dado por:

$$
\begin{array}{cc}
\text { maximizar } & U^{t} y \\
\text { sujcito a } & A^{t} y+z=c \\
& z \geq 0 .
\end{array}
$$

Un ponto é dito interior quando todlas as variáveis estão estritamente dentro

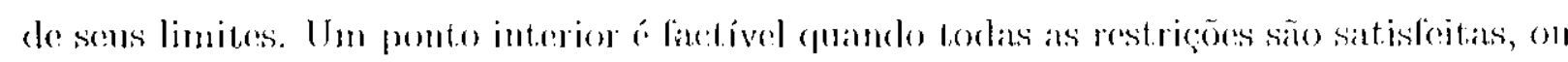
seja, o pouto $x^{0}$, onde, $A x^{0}=b$ com $x^{0}>0$ é um ponto interior lactível. As condiçoes de otimalidade dos problemas primal o dual saio formadas pela: factibilidade primal

$$
\begin{array}{r}
\text { b) } 1 x=0 \\
x \geq 0,
\end{array}
$$

factibilidade dual

$$
\begin{array}{r}
c-A^{t} y-z=0 \\
z \geq 0
\end{array}
$$

e condişoes de complementaridade $[9,24]$ :

$$
x_{i} z_{i}=0, i=1, \ldots, n
$$


(1) sigia:

$$
\text { X\%: } \cdots
$$

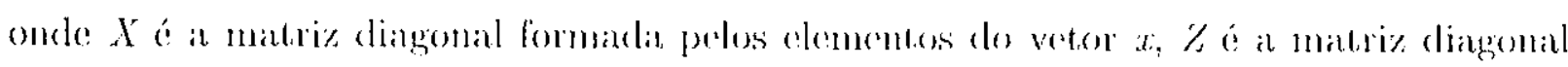

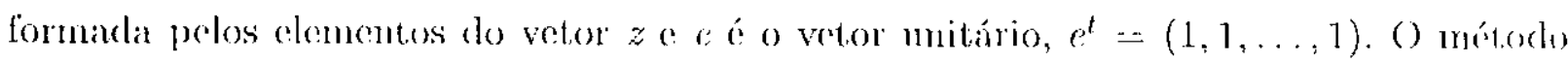
de pontos interiores prinal-dual pode ser desenvolvido atraves aplicario do médodo de Newton as condieces de otimalidarle.

\subsection{Método de Newton para Uma Variável}

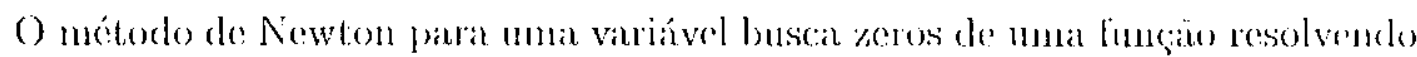

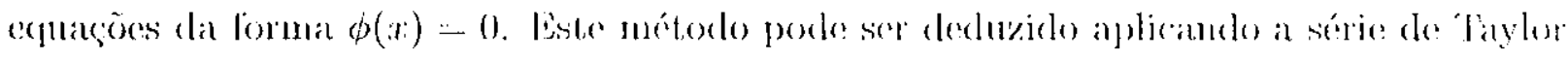
a $\phi(x)$ ent lono de $x^{0}$, obtendo:

$$
\phi(x)-\phi\left(x^{0}\right)+\phi^{\prime}\left(x^{0}\right)\left(x-x^{0}\right)+\ldots
$$

Ignorindo os termos de orem superior temest $\phi(x)=0$ :

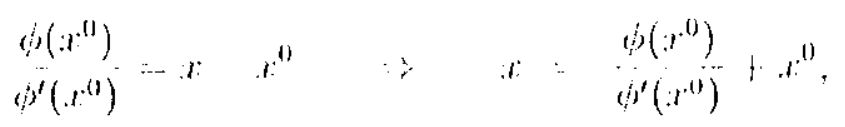

obtendo assin o processo iterativo:

$$
x^{k+1}-x^{k}-\frac{b\left(x^{k}\right)}{\left(y^{k}\left(x^{k}\right)\right.}
$$

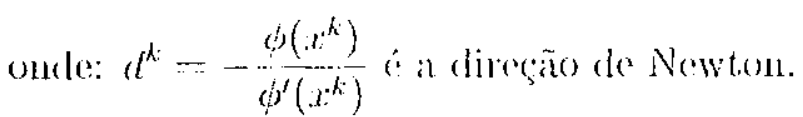

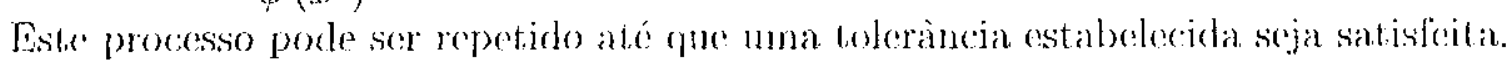

\subsection{Método de Newton para Várias Variáveis}

O mótodo de Vewton para várias variáveis tem como olejetivo concontrar os

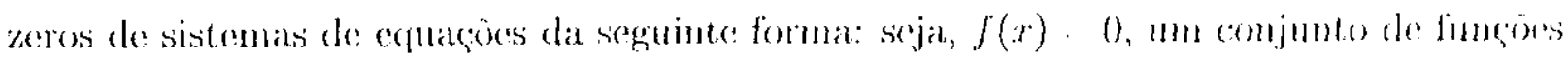
não-lineares $\phi_{i}(x)$ male $\phi_{i}(x)=0, i=1, \ldots, n$.

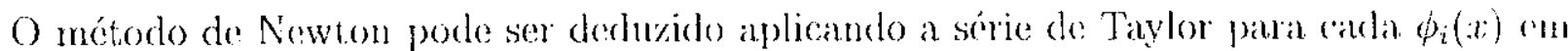


lomede de $x^{0}$, ohtendos:

$$
\phi_{i}(x)=\phi_{i}\left(x^{0}\right)+\left[\nabla \phi_{i}\left(x^{0}\right)\right]^{\prime}\left(x-x^{0}\right)+\ldots,
$$

para torlo $i=1, \ldots, n$, onde:

$$
\nabla \phi_{i}\left(x^{0}\right)=\left(\begin{array}{c}
\frac{\partial \phi_{i}\left(x^{0}\right)}{i x x^{2}} \\
\vdots \\
\frac{\partial \phi_{i}\left(x_{i}^{0}\right)}{\left.i x x^{i}\right)}
\end{array}\right)
$$

Ignorando os termos de ordem superior temos:

$$
\left[\nabla \phi_{i}\left(x^{n}\right)\right]^{t}\left(x-x^{0}\right) \cdots-\phi_{i}\left(x^{0}\right), \quad i-1, \ldots, n .
$$

Onde definimos agora o . Jacobiano no ponto $x^{0}$ :

$$
J\left(x^{0}\right)-\left(\begin{array}{c}
\nabla \phi_{1}\left(x^{0}\right) \\
\vdots \\
\nabla \phi_{n}\left(x^{0}\right)
\end{array}\right)^{\ell} \quad \text { e } \quad f\left(x^{0}\right)=\left(\begin{array}{c}
\phi_{1}\left(x^{0}\right) \\
\vdots \\
\phi_{n}\left(x^{0}\right)
\end{array}\right) .
$$

Podemos resumir assim o método de Nowton:

Darlo $x^{(0)}$

Para $k:-0,1, \ldots$

$$
\begin{aligned}
& d^{k}-J\left(x^{k}\right){ }^{1} f\left(x^{k}\right) \\
& x^{k+1}=x^{k}+d^{k}
\end{aligned}
$$

até convergir.

\subsection{Método Primal-Dual Afim Escala}

Este método cneontra a soluçáo dos problemas prinal e dual aplicando variantes do método de Newton às condiçós do otimalidade, o modificando o tamanho do passo para manter os pontos interiores. Vamos escrever as condiçoes de otimalidade da seguinte forma:

$$
f(x, y, z) \cdot\left(\begin{array}{c}
A x+b \\
A^{t} y+z-c \\
X Z z
\end{array}\right)=0 .
$$




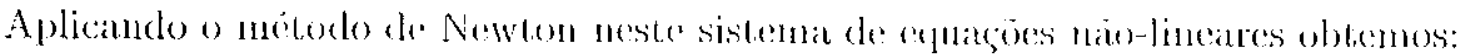

$$
\begin{aligned}
(x, y, a) & \cdots\left(x^{\prime \prime}, y^{\prime \prime}, z^{\prime \prime}\right)-\left(J\left(x^{\prime \prime}, y^{\prime \prime}, z^{\prime \prime}\right)\right)-f\left(x^{\prime \prime}, y^{\prime \prime}, z^{\prime \prime}\right) \\
& =\left(x^{0}, y^{0}, z^{0}\right) \cdot d^{0} .
\end{aligned}
$$

Seja:

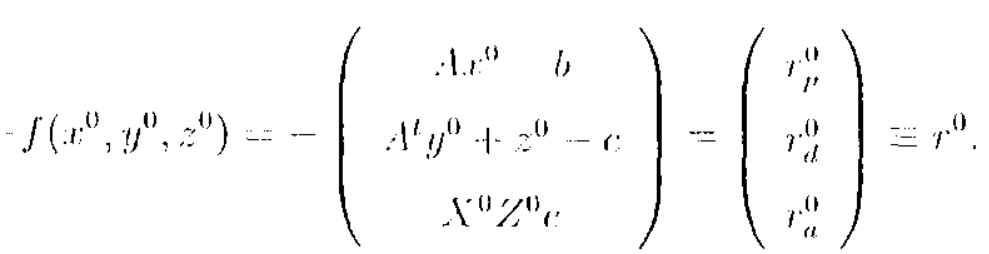

A direçäo do método de Newton aplicado às equaçoes $f(x, y, z)$ tonna-se:

$$
J\left(x^{0}, y^{0}, z^{0}\right) d d^{0}=\left(\begin{array}{ccc}
A & 0 & 0 \\
0 & A^{l} & I \\
Z^{0} & 0 & X^{0}
\end{array}\right)\left(\begin{array}{c}
d_{x}^{0} \\
d_{y}^{0} \\
d_{z}^{0}
\end{array}\right)=\left(\begin{array}{c}
r_{1}^{0} \\
r_{a}^{0} \\
r_{a}^{0}
\end{array}\right),
$$

$\log 0$ :

$$
d^{0}=\left(\begin{array}{c}
d_{x}^{0} \\
d_{y}^{0} \\
d_{z}^{0}
\end{array}\right)=\left(\begin{array}{ccc}
A & 0 & 0 \\
0 & A^{\prime} & I \\
Z^{0} & 0 & X^{0}
\end{array}\right)^{-1}\left(\begin{array}{c}
r_{p}^{0} \\
r_{t}^{0} \\
r_{a}^{0}
\end{array}\right)
$$

Podemos assim resumir o método de pontos interiores primal-dual atim escala [15]:

Dado um ponto interior $\left(x^{0}, y^{0}, z^{0}\right)$ onde $\left(x^{0}, z^{0}\right)>0$,

Para $k=0,1, \ldots$

$$
\begin{aligned}
& r_{p}^{k}=\cdots x^{k}+b \\
& r_{d}^{k}=-A^{t} y^{k}-z^{k}+c \\
& r_{a}^{k}=-X^{k} Z^{k} c \\
& d^{k}=J^{1}\left(a^{k}, y^{k}, \varkappa^{k}\right) r^{k}
\end{aligned}
$$

calcule o tamanho do passo primal $\alpha_{p}^{k}$ e dual $\alpha_{t}^{k}$

$$
\begin{aligned}
& x^{k+1}=x^{k}+\alpha_{y}^{k} d_{x}^{k} \\
& y^{k+1}=-y^{k}+\alpha_{d t}^{k} d_{y}^{k} \\
& z^{k+1}=z^{k}+\alpha_{d}^{k} d_{z}^{k}
\end{aligned}
$$

ate convergir. 
() critério de convergência usado nesse mét odo é bascado nas condiçôs de otimalidade:

$$
\begin{gathered}
\left|\frac{x^{t} z}{1+c^{\prime} z+b^{\prime} y}\right| \leq c, \\
\frac{\|b-A x\|}{1+\|b\|} \leq c, \\
\frac{\left\|c-A^{\prime} y-z\right\|}{1+\|c\|} \leq c,
\end{gathered}
$$

onde $\epsilon$ ć a tolcrancia desejada.

o tamanho do passo $\alpha_{p}^{k}, \alpha_{d}^{k}$ é calculado da scruinte forma, primeiramente calculamos:

$$
\begin{aligned}
& \rho_{p}^{k}=\min _{d x_{i}^{k}<0}\left\{-\frac{x_{i}^{k}}{d x_{i}^{k}}\right\}, \\
& \rho_{d}^{k}=\min _{d z_{i}^{k}<0}\left\{-\frac{z_{i}^{k}}{d z_{i}^{k}}\right\} .
\end{aligned}
$$

Os valores $\rho_{p}^{k}$ e $\rho_{d}^{k}$ representam o tamanlo de passo máximo tal que a primeira variável de $x$ e z se anulam respectivamente.

Logo, o tamanho do passo $\alpha_{p}^{k}$ e $\alpha_{d l}^{k}$ será multiplicado por $\tau \in(0,1)$ :

$$
\left\{\begin{array}{l}
\alpha_{p}^{k}=\min \left(1, \rho_{p}^{k}\right) \\
\alpha_{d}^{k}=\min \left(1, \rho_{d}^{k}\right),
\end{array}\right.
$$

o) que garante que nenbuma variável de $x$ on $z$ será anulada.

Os métodos afim-escala tem uma clesvantagem importante, eles permitem que as variaveis $(x, z)$ se aproximem de scus linnites muito rapidamente. Consegüentemente as direçôs calculadas são muito distorcidas e o método converge lentamente, pois $x_{i} z_{i} \approx 0$ Para evitar que isto ocorra é acrescentada uma perturbaçấo (h) na condição de complementaridade $x_{i} z_{i}=0$. Em sén lugar consideranos $x_{i} z_{i}=$ to on seja, o método primal-dual resolve o scguinte sistema de exuluçoses não-lineares a cada iteração:

$$
\left\{\begin{array}{l}
b-A x^{k}=0 \\
c-A^{k} y^{k}-z^{k}=0 \\
\mu^{k} e-X^{k} Z^{k} c=0,
\end{array}\right.
$$

onde $\mu^{k}$ ć um parâmetro que varia a cada iteração $\mu^{k} \rightarrow 0$ quando $k \rightarrow \infty$. As únicas alteraçôs do problema primal-dual clássico em relação a mótodo afim-çcala são al subs-

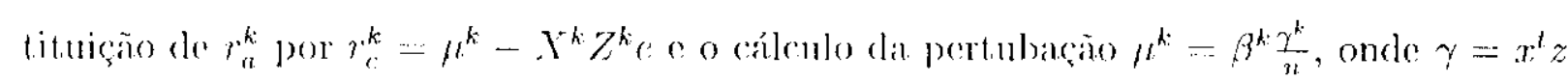


representa o gap de dualidade se $x$ e z säo factíveis $[26], \beta \in(0,1)$ e $\frac{x^{k}}{n}$ setia uma "medicla"

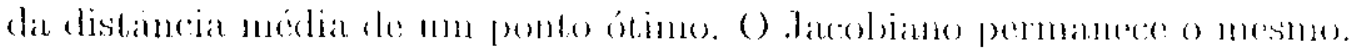

\subsection{Método Preditor-Corretor}

() mitordo preditor-corretor é o método de pontos interiores mais utilizarlo na prática, pois obtém os melhores resultados práticos e tem convergência quadrálica

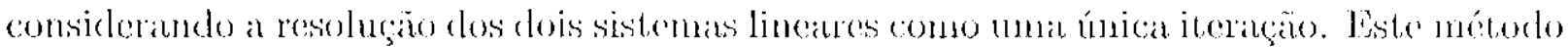

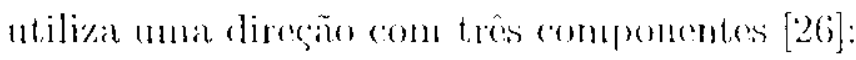

- direciano alim-escala;

- lireçio de centragem $(\beta)$;

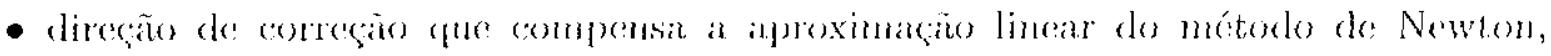
$\left(x+d_{x}\right)^{\prime}\left(z+d_{z}\right)=d_{x}^{t} d_{z}$.

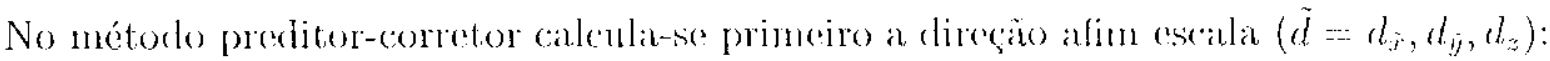

$$
\left\{\begin{array}{l}
A d_{x}=r_{p} \\
A^{\prime} d_{i y}+d_{z} \cdots r_{d} \\
y d_{i}+X d_{z}=r_{a}-X Z d
\end{array}\right.
$$

Usando o mesmo dacobiano encontrat-se enn segruda a direcio perturbata no ponto:

$$
(\tilde{u}, \ddot{y}, \ddot{z})=(x, y, z)+\left(d_{i}, d_{i j}, d_{z}\right),
$$

oll seja:

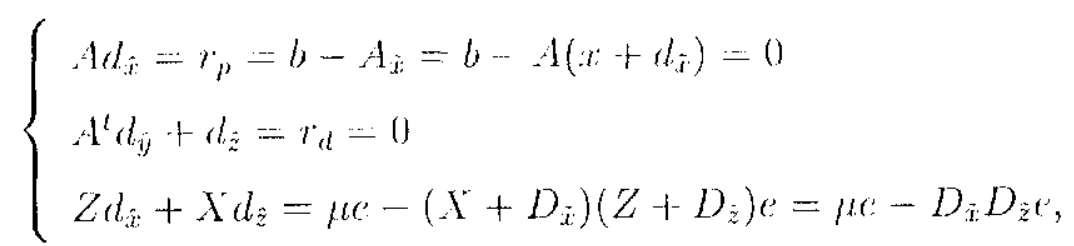

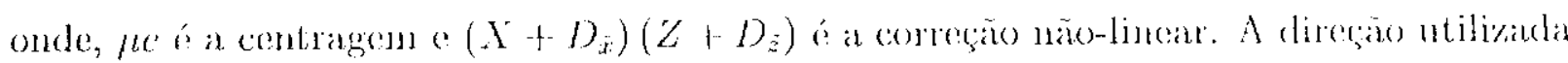
seráa a somal das duas diregoess

$$
\left\{\begin{array}{l}
d_{x}=d_{x}+d_{i} \\
d_{y}=d_{y}+d_{l} \\
d_{z}=d_{z}+d_{y}
\end{array}\right.
$$


Logo, $\left(d_{x}, d_{y}, d_{z}\right)$ podem ser alcularlos diretamente somando os dois sistomas lincares:

$$
\left\{\begin{array}{l}
A\left(d_{\tilde{x}}+d_{\hat{x}}\right)=r_{p} \\
A^{t}\left(d_{\hat{y}}+d_{\hat{y}}\right)+d_{\tilde{z}}+d_{\hat{z}}=r_{d} \\
Z\left(d_{\tilde{x}}+d_{\hat{x}}\right)+X\left(d_{\tilde{z}}+d_{\dot{z}}\right)=r_{a}+\mu e-D_{\tilde{r}} D_{\tilde{z}} C
\end{array}\right.
$$

ou seja, $\hat{d}=\left(d_{\hat{x}}, d_{\hat{y}}, d_{\hat{z}}\right)$ nunca ó calculada.

Substituindo as direçöes, temos:

$$
\left\{\begin{array}{l}
A d_{s}=r_{p} \\
A^{t} d_{y}+d_{z}=r_{d} \\
Z d_{x}+X d_{z}=r_{a}+\mu c-D_{x} D_{z} c-r_{s}
\end{array}\right.
$$

O cálculo da perturbação $\mu^{k}$ é função da direção afim. Quanto melhor a direção menor será a perturbalção e vice-versa. Sejam:

$$
\begin{aligned}
& \mu^{k}=\beta_{n}^{k} \gamma_{n}^{k}, \\
& \tilde{\gamma}^{k}=\left(x^{k}+\tilde{\sigma}_{p}^{k} d_{\hat{\gamma}}^{k}\right)^{l}\left(z^{k}+\tilde{\gamma}_{d}^{k} d_{z}^{k}\right),
\end{aligned}
$$

onde $\tilde{\sigma}_{p}^{k}$ o $\tilde{n}_{d}^{k}$ são os tamanlos rlos passos em rolacäo aos problemas primal e dual respect.ivamente. Söo calcularlos da seguinte forma:

$$
\begin{aligned}
& \tilde{\rho}_{p}^{k}-\min _{d x_{i}^{k}<0}\left\{-\frac{\tilde{n}_{i}^{k}}{d \tilde{x}_{i}^{k}}\right\}, \\
& p_{t l}^{k}=\min _{d z_{i}^{k}<0}\left\{\begin{array}{c}
\tilde{z}_{i}^{k} \\
\cdots \dot{z}_{i}^{k}
\end{array}\right\} \text {, }
\end{aligned}
$$

senclo:

$\tilde{\gamma}_{p}^{k}=\min \left(1, \tau \tilde{\rho}_{p}^{k}\right) \because \alpha_{d}^{k}=\min \left(1, \tau \dot{\rho}_{d}^{k}\right), \operatorname{com} \tau \in(0,1)$.

onde:

$$
\beta^{k}= \begin{cases}\left(\frac{\bar{\gamma}^{k}}{\gamma^{k}}\right)^{3}, & \text { se } \gamma^{k}>1 \\ \frac{\gamma^{k}}{\sqrt{n}}, & \text { caso contrário. }\end{cases}
$$

A escolha ra direção de contragem quando $\gamma^{k} \leq 1$ é bascarla en razón técricas e são mostradas em [22]. Podemos resumir o método preditor-corrotor da seguinte forma:

Dalos $\tau \in(0,1),\left(x^{0}, y^{0} ; z^{0}\right)$ tal cule $\left(x^{0}, z^{0}\right)>0$,

Pain $h=0,1, \ldots$ 


$$
\begin{aligned}
& r_{p}^{k}=b \cdots \cdot A x^{k} \\
& r_{d}^{k}=c-A^{k} y^{k}-z^{k} \\
& r_{a}^{k}=-X^{k} Z^{k} e_{e} \\
& \text { calcule } \tilde{d}^{k} \mathrm{e} \tilde{\alpha}_{p}^{k}, \tilde{\alpha}_{d}^{k}
\end{aligned}
$$

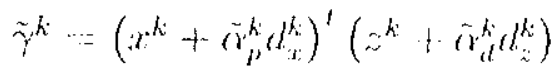

$$
\begin{aligned}
& \mu^{k}=\left(\frac{\dot{\gamma}}{\gamma}\right)^{2} \frac{\dot{y}}{b} \\
& r_{s}^{k}=\mu^{k} e+r_{a}^{k}-D_{\bar{x}^{k}} D_{\bar{z}^{k}} \cdot \\
& \text { calcule } d^{k} \text { e } \alpha_{p}^{k}, \alpha_{d}^{k} \\
& x^{k+1}=x^{k}+x_{p}^{k} d_{x}^{k} \\
& y^{k+1}=y^{k}+\alpha_{d}^{k} d_{y}^{k} \\
& z^{k+1}=z^{k}+\alpha_{d}^{k} d_{z}^{k}
\end{aligned}
$$

ató convergir.

O critério de convergêneia é o mesmo do método primal-alual alinn escalia. O mótoxio resolve dois sistemas utilizando a mesma matriz.

\subsection{Cálculo das Direções nos Métodos de Pontos}

\section{Interiores}

O sistema lincar (1.5) tem dinnensäo $2 n+m$. Este sistema pode ser reduzido à dimensão $m$ através dlas seguintes relaçón:s:

$$
\begin{aligned}
& d_{y}=\left(A D^{-1} A^{1}\right)^{-1}\left(r_{p}+A D^{-1} r_{d l}-A Z^{1} r_{s}\right) \\
& d_{x}=D^{-1}\left(A^{1} d_{y}-r_{d}+X^{-1} r_{s}\right) \\
& d_{z}=X^{1}\left(r_{s}-Z d_{s}\right) .
\end{aligned}
$$

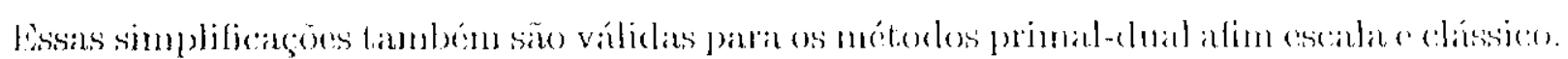

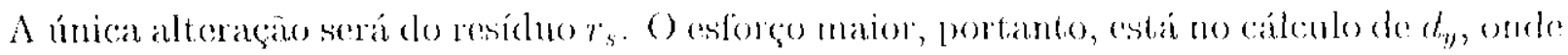

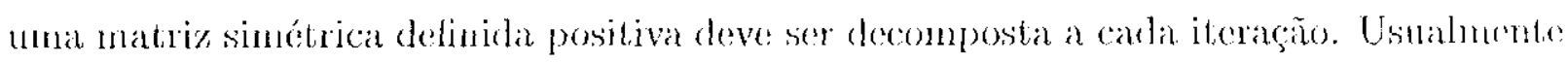
E utilizada a decomposiço de Cholesky [6] na resolucäo deste sistema lineat. 


\section{Capítulo 2}

\section{Fluxo de Carga}

\subsection{Aspectos Gerais}

O cálculo de fluxo de cargar (ou fluxo de potencia) em uma rede de energia elétrica consiste na determinaçäo do estedo da rede, da distribuiçäo dos fluxos o do algnmas outras grandezas de interesse. Nesse tipo de problema, a morlelagem do sistema é estática, significando que a rede é representada por um conjunto de equaçöes e incquaçõos algébricass. Em getal, para esse cálento, utiliza-se métodos computacionais especílicos desenvolviclos para a resohnão do sistema.

Os componemtes de $\mathrm{nm}$ sistema de emergia clétrica porlen ser classiticarlos cm dois grupos: os que estão ligados entre um nó qualquer e o nó-terra, como é o caso dos geradores, cargats, reatores o capacitores; e os que estãu ligados entre dois nós quaisquer da rede, como co o caso de linhas de transmissão, transformadores e defasadores. Os geraclores e cargas são considerados como a parte extena do sistema, e sĩo modelados atraves de injegôes de potencias nos nós da rede. A parte interna do sistema é constituída preks demais componentes $\{16\}$

As equaçoes do fluxo de carga säo obtidas imponde-se a conservação dats potências ativa e reativa en carla barra (nó) da rece, isto ó a potência líquicla injotadia deve ser ignal in soma das potoncias que fluom polos componentes intenos que têm esta barra como uma de seus terminais. Isso equivale a impor a Primeira Lei de Kirchhoff. A Segundir Lei de Kirchloff ó milizada para expressir os fluxos de potencia 
nos componentes internos como funçoes das tensões (estados) do stas batras terminais.

\subsection{Formulação do Problema}

Na formularäo mais simples, a cada barra da rede sâo associadas quatro variaveis, senclo que a lensäo serí representadia neste traballo em coorelenadas cartesiantas:

re representa a parte real da tensiáto;

$s_{k}$ representa a parte imaginária da t.ensão;

$P_{k}$ representa a geraçăo lífuida (geraçoùo menos carga) de potência ativa;

$Q_{k}$ represonta a injeção líquida de potência reativa.

O) conjunto de equaçós do problema do fluxo de carga é formado por duas equaçös para cada barra, cada uma delas representando o fato de as potencias ativas c reativas injetadas em una barra serem iguais à soma dos fluxos correspondentes que deixam a harra através de linhas de transmissino, transformadores, ce, (Primeira Lei de Kirchhoff) e pode ser expresso matematicamente como segue:

$$
\begin{aligned}
P_{k} & =\sum_{m \in \Omega_{k}} P_{k m}\left(r_{k}, s_{k}, r_{m}, s_{m}\right), \\
Q_{k}+Q_{k}^{\text {sll }}\left(v_{k}\right) & =\sum_{m \in \Omega_{k}} Q_{k m}\left(r_{k}, s_{k}, r_{m}, \dot{s}_{m}\right),
\end{aligned}
$$

onde:

$k=1, \ldots, N B$, sendo $N B$ o número de harras da rede;

$\Omega_{k} \quad$ representa o conjunto das barras vizinhas da barra $k$;

$v_{k} \quad$ representa o móslulo da tensiso da barra $k$;

$J_{k: m}$ representa of fluxo de potêneia ativa no ramo $k-m$;

$Q_{k m}$ representa o fluxo de polência reativa no ramo $k-m$;

$Q_{k m}^{s i}$ representa o componente da injeção de potencia reativa devida ao elemento shunt da barra $k$ : $\left(Q_{k}^{\text {sh }}=b_{k}^{\text {sl }} v_{k}^{2}\right.$, sendo $b_{k}^{\text {sh }}$ a susceptância shunt ligada à barra $\left.k\right)$. 


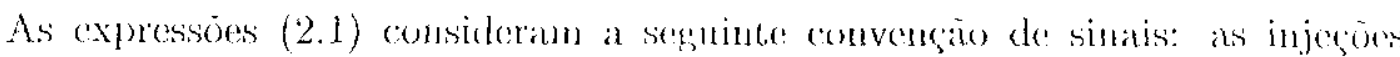
líquidas de potencia siós positivas quando futrann na barra (geraçäo) e negativas cquanelo saum da barma (carga); os fluxos de potencia são positivos quando saem da barra e negh-

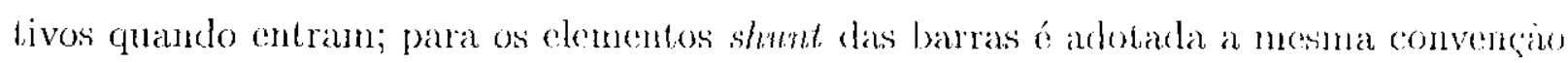
que para as injegoes.

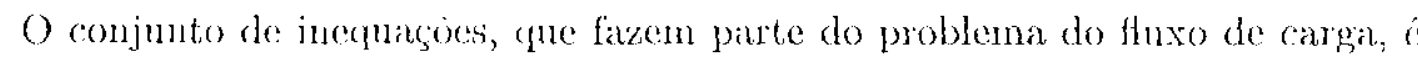
formaklo, entre outras, pelas restriçoes nats matgnitudes das tensóes nas barras de carrgat e polos linites nas injecoes de potencia reativa nas harras de controle de reativos:

$$
\begin{aligned}
& \eta_{k}^{\text {minn }} \leq v_{k} \leq v_{k}^{\text {minax }}, \\
& Q_{k}^{\text {mint }} \leq Q_{k} \leq Q_{k}^{\text {mix }} .
\end{aligned}
$$

\subsection{Modelagem}

Näo estamos considerando os transformadores defasadores, pois algumas matrizes do sistema, como a matriz adnitância, podem se tormar assimetricas, o (que nos impossibilita algumas consideraçoes e propriedades importantes para a resoluçăo do sistema (veja Capitulo 5). A consideragano de transformadores defisiatores no modelo ó trivial.

\subsubsection{Linhas de Transmissão}

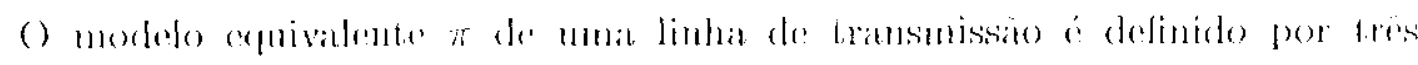

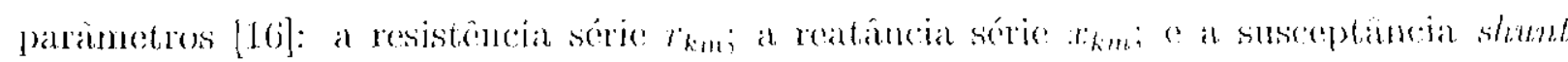

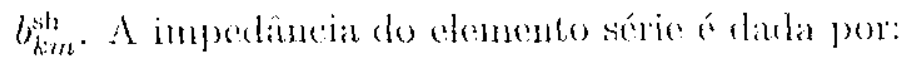

$$
z_{k m n}=r_{k m}+j m_{k m l}
$$

onde $j$ é nesta representação a unidade imaginária $(\sqrt{-1})$, encunanto a admitância série:

$$
y_{k m}=g_{k m}+j b_{k m}=z_{k m}^{-1}=\frac{r_{k m}}{r_{k m}^{2}+x_{k m}^{2}}-j \frac{x_{k m}}{r_{k m}^{2}+x_{k m}^{2}},
$$

ou seja, a condutância série $g_{k m}$ e a susceptanncia série blm são daulas por:

$$
g_{k m}=\frac{r_{k m}}{r_{k m}^{2}+x_{k m}^{2}} \quad 0 \quad b_{k m n}=\frac{-x_{k m}}{r_{k m}^{2}+x_{k m}^{2}} .
$$


Quando o modelo $\pi$ represcuta uma linha de transmissão têm-se $r_{k m}$ c $x_{k m}$ positivos, o que implica $g_{k m}$ positivo e $b_{k m}$ negativo (tipo indutivo). Já o elemento $b_{k m}^{\text {sh }}$ é positivo, pois o shunt é do tipo capacitivo.

A corrente $I_{k \cdot m}$ ó formada de uma componente série e uma componente shunt, e pode ser calculada a partir das tensões fasoriais terminais $E_{k}$ e $E_{m m}$, e dos parâmetros do modelo equivalente $\pi$ :

$$
I_{k m}=y_{k m}\left(E_{k}-E_{m}\right)+j b_{k m}^{\mathrm{sh}} E_{k}
$$

onde:

$$
E_{k}=r_{k}+j s_{k} \quad \text { e } \quad E_{m}=r_{m}+j s_{m}
$$

Analogancente, a corrente I Im é darla por:

$$
I_{m k}=y_{m k}\left(E_{m}-E_{k}\right)+j l_{k m}^{\mathrm{sh}} E_{m}
$$

\subsubsection{Fluxos de Potência Ativa e Reativa}

As expressões dos fluxos de potência ativa $P_{k m}$ e potência reativa $Q_{k m}$ podem ser obtidas a partir dos modelos apresentados na seçăo 2.3.1, conforme será mostrado a seguir.

Sabemos que a corronte $I_{k m}$ em uma linha de transnissão ć dada por (2.5). Substituindo por $(2.3)$ o (2.6) temos:

$$
\begin{aligned}
I_{k m}= & y_{k m}\left(E_{k}-E_{m}\right)+j b_{k m}^{\mathrm{sh}} E_{k} \\
= & \left(r_{k}+j s_{k}-r_{m}-j s_{m}\right)\left(g_{k m}+j b_{k m}\right)+j b_{k m}^{\mathrm{sh}}\left(r_{k}+j s_{k}\right) \\
= & r_{k} g_{k m}+j r_{k} b_{k m}+j s_{k} g_{k m}-s_{k} b_{k m}-r_{m} g_{k m}-j r_{m} b_{k m}-j s_{m} g_{k m}+s_{m} b_{k m} \\
& +j r_{k} b_{k: m}^{\mathrm{sil}}-s_{k} b_{k m}^{\mathrm{sh}} .
\end{aligned}
$$

O) fluxo de potência complexa correspondente é:

$$
\begin{aligned}
S_{k m}^{*}= & P_{k m}-j Q_{k m}=E_{k}^{*} I_{k m} \\
= & \left(r_{k}-j s_{k}\right)\left[\left(r_{k}+j s_{k}-r_{m}-j s_{m}\right)\left(g_{k m}+j b_{k m}\right)+j b_{k m}^{\mathrm{sh}}\left(r_{k}+j s_{k}\right)\right] \\
= & r_{k}^{2} g_{k m}-r_{k} r_{m} g_{k m}+r_{k} s_{m} b_{k m}+s_{k}^{2} g_{k m}-s_{k} s_{m} g_{k m}-r_{m} s_{k} b_{k m} \\
& -j\left(-r_{k}^{2} b_{k m}+r_{k} r_{m} b_{k m}+r_{k} \cdot s_{m} g_{k m}-s_{k}^{2} b_{k m}+s_{k} s_{m} b_{k m}-r_{m} s_{k} g_{k m}\right. \\
& \left.-r_{k}^{2} b_{k m}^{\mathrm{sh}}-s_{k}^{2} b_{k m}^{\mathrm{sh}}\right) .
\end{aligned}
$$




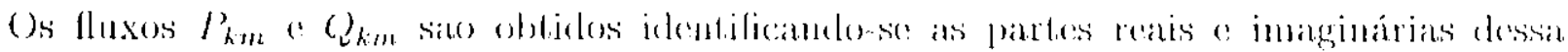
expagiono comploxa, resultando:

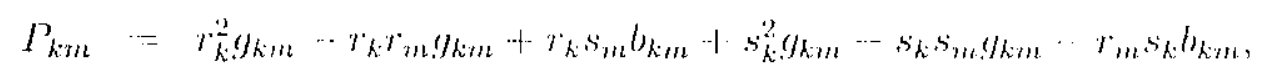

$$
\begin{aligned}
& Q_{k m}=-r_{k}^{2} b_{k m i}+r_{k} r_{m} b_{k m n}+r_{k} s_{m} g_{k m}-s_{k}^{2} b_{k m}+s_{k} s_{m} b_{k m}-r_{m} s_{k} g_{k m} \\
& \left.-r_{k}^{2} b_{k m}^{s h t}-s_{k}^{2} b_{k m}^{s h}\right)
\end{aligned}
$$

Os fluxos $P_{m k}$ e $Q_{m k}$ sĩo obticlos de forma análogia:

$$
\begin{aligned}
P_{m k}= & r_{m}^{2} g_{k m}-r_{m} r_{k} g_{k m}+r_{m} s_{k} b_{k m}+s_{m}^{2} g_{k m}-s_{m} s_{k} g_{k m}-r_{k} s_{m} b_{k m}, \\
Q_{m k}= & -r_{m}^{2} b_{k m}+r_{m} r_{k} b_{k m}+r_{m} s_{k} g_{k m} \cdots-s_{m}^{2} b_{k m}+s_{m} s_{k} b_{k m}-r_{k} s_{m} g_{k m} \\
& \left.-r_{m}^{2} b_{k m}^{\mathrm{sh}}-s_{m}^{2} b_{k m}^{\mathrm{sh}}\right) .
\end{aligned}
$$

As perdas de potencia ativa e reativa na linha sio dadas, respectivanente, por:

$$
\begin{aligned}
P_{k m}+P_{m k}= & g_{k m}\left(r_{k}^{2}+s_{k}^{2}+r_{m}^{2}+s_{m}^{2}-2 r_{k} r_{m}-2 s_{k} s_{m}\right), \\
Q_{k m}+Q_{m k}= & b_{k m}\left(-r_{k}^{2}-s_{k}^{2}-r_{m}^{2} \cdots s_{m}^{2}+2 r_{k} r_{m}+2 s_{k} s_{m}\right) \\
& +1 b_{k m}^{s / n}\left(-r_{k}^{2} \cdots s_{k}^{2}-r_{m}^{2}-s_{m}^{2}\right) .
\end{aligned}
$$

\subsection{Formulação Matricial}

A injegano liquida de corrente na barra ki pode ser obtida aplicando-so a Primeirat Tei de Kirchhofl para cesse modelo:

$$
I_{k}+I_{k}^{s h}=\sum_{m \in \Omega_{k}} I_{k m n}(k=1, \ldots, N B) .
$$

A corrente $I_{k m}$ en mma linha do transmiswão de dada pela seguninte expressião:

$$
I_{k: m}=\left(y / m+j b_{k m}^{s h}\right) H_{k}+\left(-y_{k m}\right) E_{m}^{1}
$$

A corrente $I_{k m}$ pode ser posta, muma forma geral [16]:

$$
I_{k m}=\left(a_{k m}^{2} y_{k m}+j b_{k m}^{\mathrm{sh}}\right) I_{k}+\left(-\cdots a_{k m} \mathrm{e}^{-j \varphi_{k m}} y_{k m}\right) l_{m}^{\prime}
$$

sendo que, para linbas de transmissão, $a_{k m}=10 \varphi_{k m}=0$; para transformadores cm-lase,

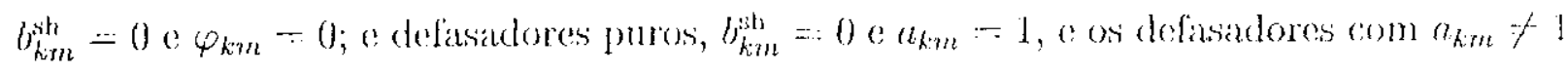


säo representados com um defasador puro $\left(a_{k m}=1\right)$ cm séric com um transformador em-fase $\left(\varphi_{k m} \cdots: 0\right)$

Consiclerando $I_{k m}$ dado em $(2.17)$ a expressão de $I_{k}(2.15)$ pode ser rescrita da seguinte mancira:

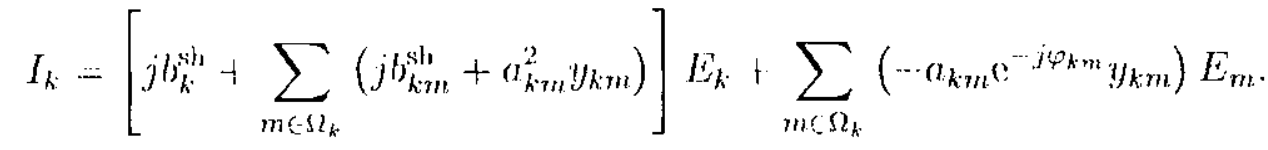

Lista expressäo, para $k=1, \ldots, N B$, pode ser representadia na forma matricial:

$$
I=Y \underline{E}
$$

onde:

I. representa o vetor das injeçós do corrente, cujas componentes são $I_{k}(k=1, \ldots, N B)$;

lí representa o votor das tensōes nodais, cujas componentes säo $E_{k}$;

$Y^{*}=G+j B$ representa a matriz admitância nodal.

Os clementos da matriz Y, consiclerando os valores para linhas de transmissão, saio:

$$
\begin{aligned}
& Y_{k: m}=-\cdots y_{k m} . \\
& Y_{k \cdot k}=j b_{k:}^{\mathrm{hl}}+\sum_{m \in \Omega_{k}}\left(j l_{k m}^{\mathrm{s} 1}+a_{k m}^{2} y_{k m}\right) .
\end{aligned}
$$

Lin geral, essa matriz ó esparsa, pois $Y_{k m}=0$ sempre que entre as barras $k$ o $m$ não exislirem linhas. Como naio estiamos considerando a presença de transformalores defasadores, a matriz $Y$ é simétrica, assim como $G$ e $B$. Aproveitamos essa propriedade na modelagem (ver Seçäo 2.3) e na resolıção do sistema (ver Seção 5.1 ).

$\Lambda$ injeção do corrente $I_{k}$, que ś a k-ésima componente do vetor $I$. pode ser colocada na forma:

$$
I_{k}=I_{k k} L_{k}+\sum_{m, C \Omega_{k}} Y_{k m} E_{m}-\sum_{m \in K} Y_{k m} E_{m}
$$

cm cue $K$ co o conjunto de todlas as barras $m$ adjacentes à barra $k$, inclusive a própria, ou seja, o conjunto $K$ ć formado pelos elementos do conjunto $\Omega_{k}$ mais a própria barra $k$. 


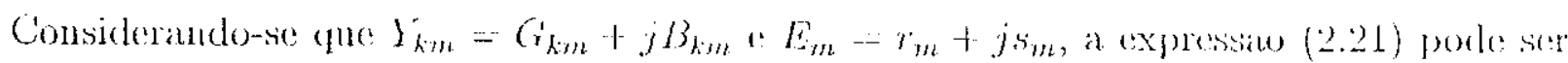
escrita da seguinte maneirat

$$
I_{k}=\sum_{m \in K}\left(C_{k m+}+j B_{k m}\right)\left(r_{m}+j s_{m}\right) .
$$

A injeção de potencial complexa $S_{k}$ é:

$$
S_{k}^{*}=P_{k}-j Q_{k}=E_{k}^{*} I_{k}
$$

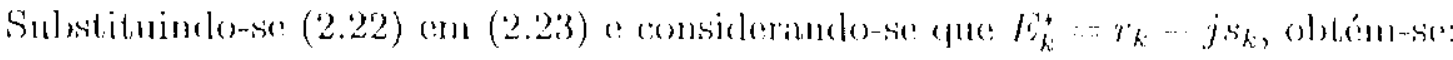

$$
S_{k}^{*}-\left(r_{k}-j s_{k}\right) \sum_{m k}\left(C_{k m m}+j B_{k m}\right)\left(r_{m}+j s_{m}\right) .
$$

As injecôes de potencia ativa e reativa podem ser obtidas identificande-se as partes raal (e) imaginániar elar expressito (2.24):

$$
\begin{aligned}
& P_{k}=\sum_{k \in k}\left(r_{k} G_{k+m} r_{m} \cdots r_{k} \cdot B_{k m} s_{m}+s_{k}\left(G_{k m n} s_{m}+s_{k} \cdot B_{k: m} r_{m}\right),\right. \\
& Q_{k}=\sum_{k \in K}\left(-s_{k} B_{k m_{L}} s_{m}-r_{k} G_{k m m} s_{m}-r_{k} B_{k m} r_{m}+s_{k} G_{k m i} r_{m}\right),
\end{aligned}
$$

generalizando para a format matricial:

$$
\begin{aligned}
& \eta=R G r+S G s+S B r-R B s, \\
& q=S G r-R G s-R B r-S B s,
\end{aligned}
$$

onde:

p representa o vetor de geração de potencia ativa, cujas componentes sĩo $P_{k}$;

I representa o velor do gerango de potencia reativa, cujas componentes são $Q_{k}$;

$r$ representa o vetor da parte real clas tensöes, cujas componentes são $r_{k}$;

s representia o vetor (la parte imaginália das tensües, cujas componentes săo sk;

$R$ represental a matriz diagonal der $r$

$S$ representat a matriz diagonal de s;

$G$ representat a matriz rle condutancial;

$B$ represental a matriz de susceptancia. 
Esta noliç̧o malricial será utilizarla na formulaçäo do problema de fluxo de: carga ótinue AC (ver Seçio 3.2)

A notação das variáveis nesses primeiros capítulos é a mesma utilizada nos livtos e arligos cilados anteriomente, pois são fundamentadlas para que se teula uma leitura didática e comprecnsiva. A partir do próximo capítulo, apresentaremos una notaçüo diferente para algumas variáveis. Esta mudança se deve à alguns conflitos de nomes de variáveis devido a tradução do texto para a implementação computacional. l'ara que nào baja quaiscuer dúviclas ma leitura dos capítulos seguinles, debalharemos as representações utilizadas nas próximas equaçõos quando necessátio. 


\section{Capítulo 3}

\section{Problema de Fluxo de Carga Ótimo}

\section{$\mathrm{AC}$}

() problema de llaxo de carga ólimo AC ć mun dos mais importantes na área de sistemas de potencia, servindo como base piana diversas outras aplicaçōes. Tha dificuldade que este problema apresenta é instabilidade numórica proveniente dos métodos de solução tradicionais. Os métodos de pontos interiores trouxeram à tona uma nova linha de pesquisa na área de sistemas de potencia $[8,14,18,17,19,20,23,25,27]$.

Esses mótodos săo reconbecidos atualmente por sula robuste\% $[14,20]$. Além disso, o tratamento eficiente de desigualdades permite uma revisio des procedinentess geralmente adotados. Assim, a utilizasäe de coordenadas cartesianas surge como uma

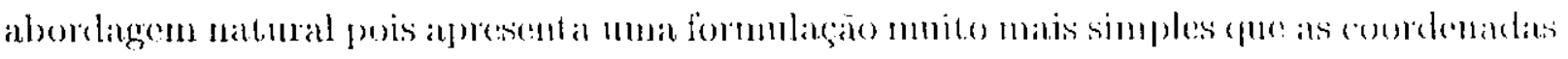
polares.

\subsection{Motivação}

$\Lambda$ tensâo (complexa) da batra (É) é definida em coordenadas cartesianas como:

$$
\underline{F}=r \cdot+j s,
$$

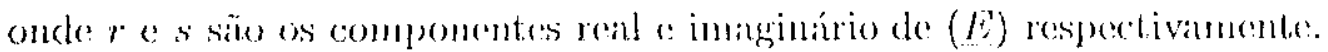

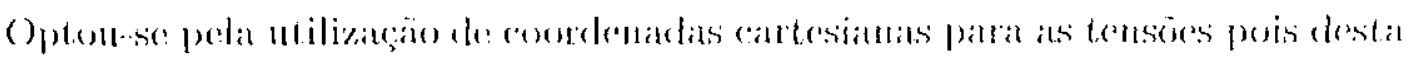
forma, tanto als restriçoes do problema como as funçoes objeljos porventura artotadas säo 
quadráticas. Conseqüientemente. as malrizes do prolsema são mais fáceis de trabalhar eo cálculo do termo de correcia do método preditor-corretor pode ser feito de forma menos custosa do ponto de visia computacional. Outra vantagem ć que a Hessiana clo problema 6́ constante r a expansào em Taylor é exata para o termo de ordem dois.

Tensöes na forma cartesiana são usadas por exemplo, para explorar a idéia de mon multipliador ótimo que melhora a convergeneia do fluxo de carga para estudos de. estimativa do estado do sislema de potencia [23].

Finalmente, a vantagen com se trabalhar com coordenadas polares, que modelam mais facilmente os limites de magnitude de tensão, perde importância devido ao tratamento de desigualdades effeiente proporcionalo pelos mélodos de pontos interiores $[14,20]$.

\subsection{Formulação do Problema}

O problema de fluxo de carga ótimo com coordenarlas cartesianas pode então

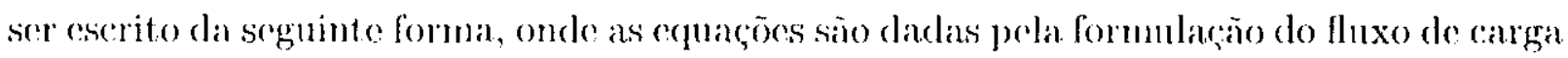
apresentado anteriormente a pela representanäo do fluxo entre as linhas do transmissão o

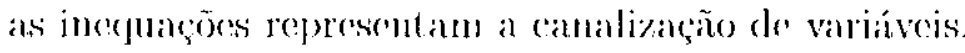

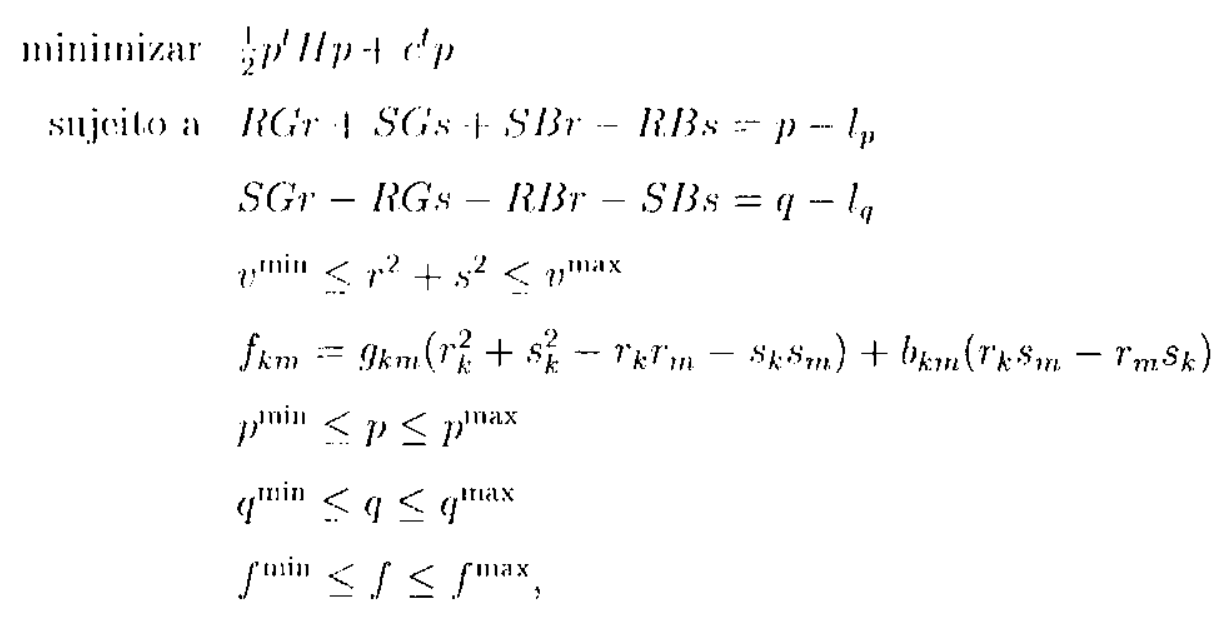

ondr:

$G$ representa a matriz de coudutîncia:

13 representa a matriz do suserplinemial

p representa a geração do potência ativa: 
q representa a geraçăo de potencia reativa;

$f_{k m}$ representa o liuxo de polencia ativa da linha ki para a linha $m$;

$H$ matriz diagonal representando o termo culadrático do custo de geraçäo;

c representa a componente lincar do casto de geraçio;

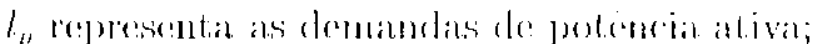

lg representa as demandas de potenciat reativa;

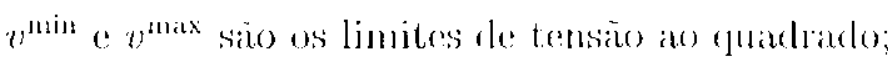

$p^{\min }$ e $p^{\max }$ são os limites de geração de potencia ativa;

$q^{\text {tuin }}$ e $q^{\text {max }}$ sĩo os linites de geraçấo de potencia reativat

$f^{\text {muin }}$ e fuax sĩo os limites de fluxo de potência ativa.

A minimização das perdas na geração é utilizada como critério de otimização Estas perdas podem ser modeladas como uma função quadrática separável tanto para geradores térmicos representando os custos, como hidrelétricos representando as perdas [21]. Vale ressaltar que outras funções objetivo podem ser adotadas sem muilas alleraçós no desenvolvimento apresentado a seguir.

\subsubsection{Problema Relaxado}

Podemos considerar o problema de fluxo de carga ótimo sem explicitar as restriçoes de limite de fluxo de potencia ativa entre as linhas de transmissão. Essa restriçöes podem ser controladas implicitumente pelo linite de geração de potência ativa. $\Lambda$ formulação a seguir representa o problema de fluxo de carga ótimo relaxando as restriçós e limites relacionados com fluxo de potencia ativa entre as linhas de transmissĩo.

$$
\begin{aligned}
& \text { minimizar } \frac{1}{2} p^{t} H p+c^{t} p \\
& \text { sujeito a } R G r+S G s+S B r-R B s=\eta \cdots l_{p} \\
& S G r-R G s-R B r+S B s=q-l_{q} \\
& v^{\text {min }} \leq r^{2}+s^{2} \leq v^{\text {minax }} \\
& p^{\text {minin }}<p \leq p^{\text {minax }} \\
& q^{\text {min }} \leq q<q^{\text {max }}
\end{aligned}
$$

Com o objetivo de simplificar a implementação computiacional, essa formulaçito 
sera utilizadia para o desenvolvimento de 1 m método específico para esse problema, que

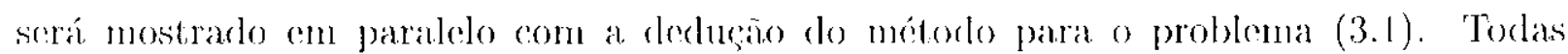
as elapas e operaçoes reatizadas no médodo serão executarlas e mostradas para ambat formulaçós.

\subsection{Simplificação da Matriz Hessiana}

No mesmo espirito de obter Hessianas mais fúceis de trabalhat ntilizando as coordenadas cartesianas, opton-se por acrescentar a restrição:

$$
r^{2}+s^{2}=v
$$

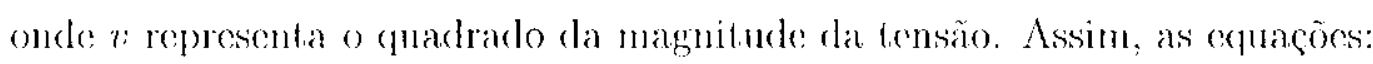

$$
\begin{gathered}
v^{\min } \leq r^{2}+s^{2} \leq v^{\operatorname{minx}} \\
f_{k m n}=g_{k m}\left(r_{k}^{2}+s_{k}^{2}-r_{k} r_{m}-s_{k} s_{m}\right)+b_{k m}\left(r_{k}, s_{m} \cdots r_{m}, s_{k}\right)
\end{gathered}
$$

são substituídas por:

$$
\begin{gathered}
v^{\min } \leq v \leq v^{\max } \\
f_{k m}=g_{k m}\left(v^{2}-r_{k} r_{m}-s_{k} s_{m}\right)+b_{k m}\left(r_{k} s_{m}-r_{m} s_{k}\right),
\end{gathered}
$$

transformando $u$ m conjunto de restrições en canalização de variáveis e simultaneamente, simplificando outro conjunto de restriçóes.

Apesare deste modelo conter um conjunto adicional de varjáveis e resitriçoes, as derivadas são mais simples a Hessiana obtida no desenvolvimento dos mótodos de pontos interiores mais esparsa. Väo se pode, entredanto, afimar de antemán se este modelo leva

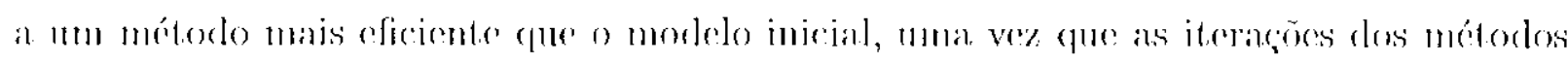
desenvolvirlos para ambos modelos peram diregoes diferentes [12]. Fe necessário realizar

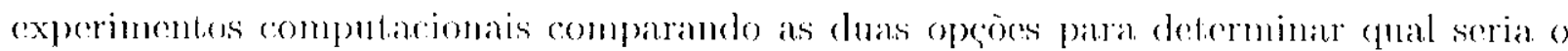
melhor morlelo. 


\section{Capítulo 4}

\section{Desenvolvimento do Método}

\subsection{Metodologia}

As técnicas utilizadas para este problema são específicas de programaçäo nãolinear, diferentemente das técnicas utilizadas em programasão linear (Capítulo 1). A aplicação do método de Newton as condiçôes de otimalidade leva a um método de pontos interiores primal-dual específico paria este modelo. As condiçoes de otimalidade por sua vez podem ser obtidas através da função lagrangiana do problema onde as restrições de desigualdade sĩo representadas jor funçoes de barreira logarítnicas das variáveis de folga.

Para simplificar o desenvolvimento do método, vamos considerar um problema onde cada barra pode gerar potência ativa e reativa e está dirctanente conectada a todas as outras barrats. Desta forma, a representacaio do fluxo entre ats linbas pode ser escritat da seguinte forma:

$$
f \therefore V G-R G R-S G S+R B S-S B R .
$$

Unar ver desenvolvido o método, bastia considerar os elementos de $f$ gue ropresentam linhas de transmissĩo existentes no sistemia em estudo preservando assinn a esparsidacle do problema.

Na prática é muito commun resolver problemas desconsiderando os fluxos nas linhas de transmissão, pois is restriçoes têm utilidade somente para verificar as capaci-

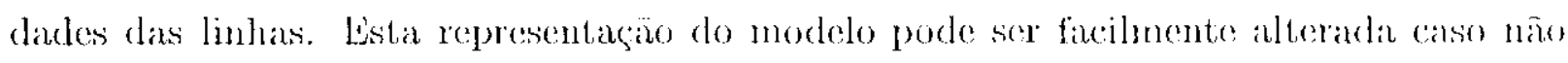
existam muitas linhas carregadas. A implenentacão computacional feitia moste trabalho 
6 bascada na representaçäo do modelo para o problema relaxado, isto é, sem as restriçōes de fluxos de potência ativa nas linhas de transmissão. O desenvolvimento do método é similar para ambos os problemas.

Com o objetivo de reduzir o numero de variáveis do problema, antes de construir a função lagrangiana, vamos fazer uma mudança de variáveis de tal forma que todos os limites inferiores das variáveis candizadas sejam anulados. O modelo adotado pode ser então escrito da seguinte forma, onde as variáveis de folga para os limites superiores taunbém são acrescentiadas:

$$
\begin{array}{ll}
\text { minimizar } & \phi(p) \\
\text { sujeito a } & R G r+S G s+S B r-R B s-p=-l_{p} \\
& S G r-R G s-R B r-S B s-q=-l_{q} \\
& V G-R G R-S G S+R B S-S B R-f=l_{f} \\
& r^{2}+s^{2}-v=l_{p} \\
& p+s_{p}=p^{\max } \\
& q+s_{q}=q^{\max } \\
& f+s_{f}=f^{\max } \\
& v+s_{v}=v^{\text {max }} \\
& \left(p, q, f, v, s_{p}, s_{q}, s_{f}, s_{v}\right) \geq 0,
\end{array}
$$

oude $\phi(p)=\frac{1}{2} y^{t} I f p+c^{t} p$ e, por abuso de notação, utilizamos os mesmos símbolos para representar os vetores antes e depois das mudanças de variáveis.

\subsection{Função Barreira Logarítmica}

Fistiamos trabalhaudo com $11 m$ problema não-linear, por isso não tomamos o problema dual de (4.1) como em programaço linear, mas utilizamos técnicas específicas para esse tipo de problema.

No problema (4.1), as restriçoos de desigualdade são as condições de não-

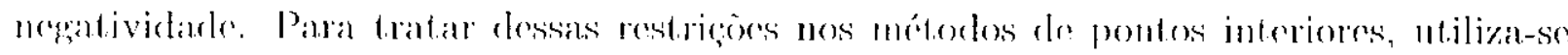




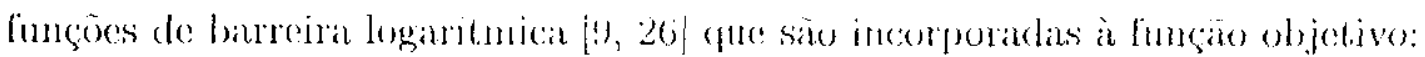

$$
\begin{aligned}
& \text { minimizar } \quad \phi(p)-\mu^{k} \sum_{i=1}^{n} \ln \left(x_{i}\right) \\
& \text { sujeito a } R G r+S C S+S B r-R B B s-p=-l_{p} \\
& S G r-R G s-R B r-S B s-q=-l_{q} \\
& V G-R G R-S G S+B B S-S B B-f=l_{j} \\
& r^{2}+s^{2}-v=l v \\
& p+s_{p}=p^{\mathrm{n} \max } \\
& q+s_{q}=q^{111 a x} \\
& f+s_{j}=f^{m+2 x} \\
& v+s_{v}=\eta^{\text {111ix }} \text {, }
\end{aligned}
$$

onde $x=\left(p, q, f, v, s_{p}, s_{q}, s_{f}, s_{v}\right)$, w ́ a dimensão do vetor $x$ o $\mu^{k}>0$ é o paràmetro do barreira que ó monotonicamonte decrescente e converge para zero durante o progressos

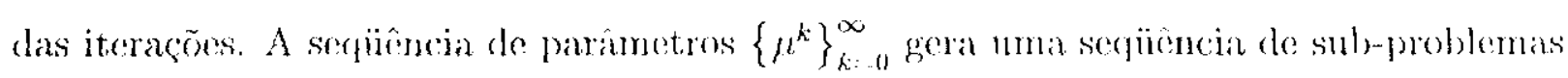

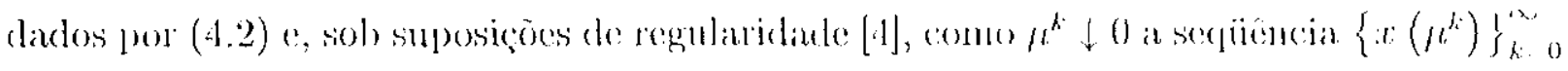
de soluçoes de (4.2) aproxinat-se de $x^{*}$, am mínimo local de (4.1) [2:3].

\subsection{A Função Lagrangiana}

A função lagrangiana $L$ das restriçốs de igmaldade do problema (4.2) é dada por [9]:

$$
L(r, s, x, l)=\phi(p)-\mu^{k} \sum \ln \left(x_{i}\right)+l^{t} L_{d d}(x),
$$


onde $t^{t}=\left(y_{p}, y_{q}, y_{f}, y_{n}, w_{p}, w_{q}, w_{f}, w_{\eta}\right)$ representa os multiplicadores de Lagrange (ou variáveis (luais) e,

$$
L_{d}(x)=\left(\begin{array}{c}
R G r+S G . s+S B r-R B s-p+l_{p} \\
S G r r-R G S \cdot R B r-S B s-q+l_{q} \\
V G-R G R-S G S+R B S-S B R-f-l_{f} \\
r^{2}+s^{2}-v \cdot l_{v} \\
p+s_{p}-p^{\max } \\
q+s_{q}-q^{\max } \\
f+s_{f}-f^{\max } \\
v+s_{v}-v^{\max }
\end{array}\right) .
$$

Jin mínino local de (4.2) é expresso em temos de um ponto estacionário de $L$, tendo que sibtisfazer as condições necessárias de primeira ordem de Karush-Kuhn-Tucker (KKT), $\nabla_{(r, s ; x, l)} L=0$, ou seja, $\nabla_{l} L=L_{d}(x)=0 \mathrm{e}:$

$$
\begin{aligned}
& \nabla_{p} L=H p+c-\mu P^{-1} c-y_{p}+w_{p}, \\
& \nabla_{\eta} L=\cdots \mu Q^{-1} \rho-y_{q}+w_{\eta}, \\
& \nabla_{f} L_{i}=-\mu F^{-1} e-y_{f}+w_{f}, \\
& \nabla_{v} L=G y_{f}-\mu V^{\cdots 1} e-y_{v}+w_{v}, \\
& \nabla_{r} L=\nabla_{r} L_{p}^{t} y_{p}+\nabla_{r} L_{q}^{t} y_{q}+\nabla_{r} L_{f}^{t} y_{f}+2 R y_{n}, \\
& \nabla_{s} L=\nabla_{s} L_{p}^{l} y_{p}+\nabla_{s} L_{q}^{l} y_{q}+\nabla_{s} L_{f}^{l} y_{f}+2 S y_{v}, \\
& \nabla_{s p} L=-\mu S_{p}^{-1} c+w_{p}, \\
& \nabla_{s q} L=-\mu S_{q}^{-1} c+w_{q}, \\
& \nabla_{s f} L=-\mu S_{f}{ }^{1} C+w_{f}, \\
& \nabla_{s v} L=-\mu S_{v}^{-1} c+w_{n},
\end{aligned}
$$

onde:

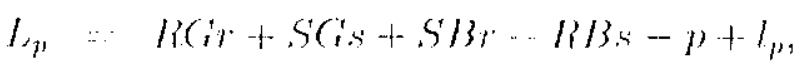

$$
\begin{aligned}
& L_{u_{1}}-S G r-R G S-R B B+S B S q+l_{q}
\end{aligned}
$$

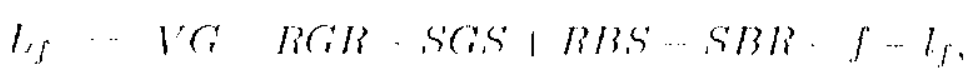


assim:

$$
\begin{aligned}
& \nabla_{r} L_{p}^{t}=G R+\operatorname{diag}(G r)+B S-\operatorname{diag}(B s), \\
& \nabla_{r} L_{q}^{l}=G S-\operatorname{diag}(G s) \cdots B R-\operatorname{diag}(B r), \\
& \nabla_{s} L_{p}^{\ell}=G S+\operatorname{diag}(G s)-B R+\operatorname{diag}(B r), \\
& \nabla_{s} L_{q}^{\ell}=-G R+\operatorname{diag}(G r)-B S-\operatorname{diag}(B s) .
\end{aligned}
$$

E para $\nabla_{r} L_{f}^{\ell}$ e $\nabla_{s} L_{f}^{\ell}$ considere o termo $\tilde{L}=$ RCS onde Có uma matriz conslante. Funtoa:

$$
\begin{aligned}
& \frac{\partial \tilde{L}}{\partial r_{i}}=\left[\begin{array}{l}
\operatorname{diag}\left(C e_{i-1} S_{i-1}\right) \\
\operatorname{diag}\left(C e_{i} S_{i}\right) \\
\operatorname{diag}\left(C e_{i+1} S_{i+1}\right)
\end{array}\right], \\
& \frac{\partial \tilde{L}}{\partial s_{i}}=\left[\begin{array}{l}
\left(C E_{i-1}\right)^{\prime} R \\
\left(C L_{i}\right)^{t} R \\
\left(C E_{i+1}\right)^{t} R
\end{array}\right],
\end{aligned}
$$

onde $E_{i}=\operatorname{diag}\left(e_{i}\right), i=1, \ldots, N B$.

Com isso podemos construir as expressóses de $\nabla_{r} L_{f}^{t}$ e $\nabla_{s} L_{f}^{t}$, pois $L_{f}$ é composto de combinações de termos similares a $\tilde{L}$, se ignorarmos açueles independentes de $r$ e s. Alén dessas relaçoes, devemos tor $x \geq 00$ (que implical $\left(w_{p}, w_{q}, w_{f}, w_{n}\right) \geq 0$.

Para obter um mótodo estritamente primal-rual resta ainda definir ats variáveis de folga duais:

$$
\begin{aligned}
& z_{p}=\mu P^{-1} e, \\
& z_{q}=\mu Q^{-1} e, \\
& z_{f}=\mu F^{\cdots 1} c_{;} \\
& z_{v}=\mu V^{-1} e
\end{aligned}
$$

Estas variáveis tambrém são não-negativas por definirgão.

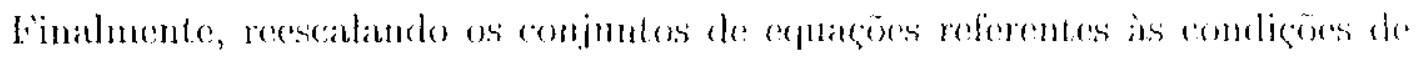
complementaridate obtemos o seguinte sistema nato-linear que comesponde is condjoues 
de otimalidade de primeria ordem do problema (1.1):

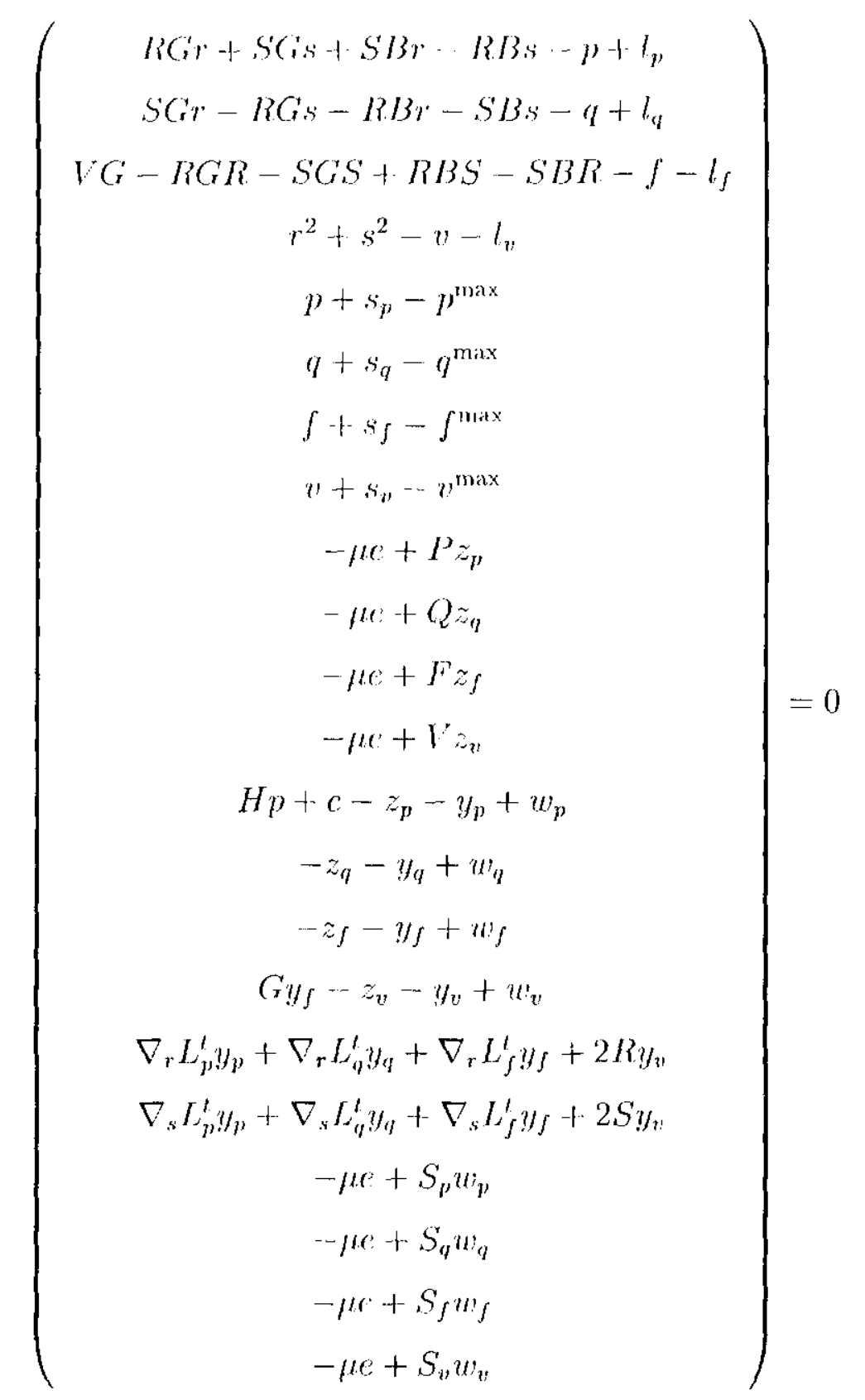

$\operatorname{com}(x, t) \geq 0$ onde $t=\left(z_{p}, z_{q}, z_{f}, z_{v}, w_{p}, w_{q}, w_{f}, w_{v}\right)$ 


\subsection{Eliminação de Variáveis Livres y}

É possived aplicas o método de pontos interiores primal-dual rlimetamente an sistema linear (4.5), no entanto, parece ser mais aconselhável eliminar as variaveis y do

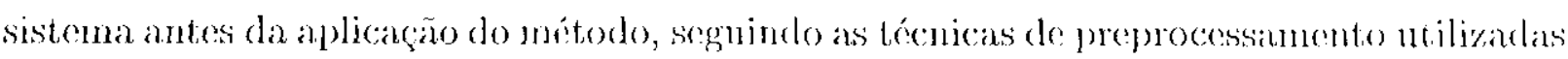

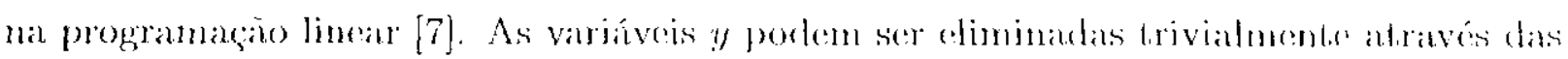
equacoes:

$$
\begin{aligned}
& y_{p}=H_{p}+\cdot \cdot-z_{p}+m_{p} \\
& y_{4}=w_{i}-z_{q}, \\
& y_{f}=w_{f}-z_{f}, \\
& y_{v}=G y_{j}-z_{v}+w_{v}=C y_{f}+\tilde{y}_{n} .
\end{aligned}
$$

Estas eliminaçôs podem ser feitas porque as vajáveis duais y sĩo irrestribas. Além disso, una ver que (stas climinações são triviais, a estrutura esparsa do sistema linear não se altera.

Eimportante notar que um método de pontos interiores aplicalo ao sistema (4.5) sem a elininagão de y é diferente de un método aplicando ao mesno sistema näolinear eliminando as variáveis y. É necessária nun comparagio nunérica entre os dois

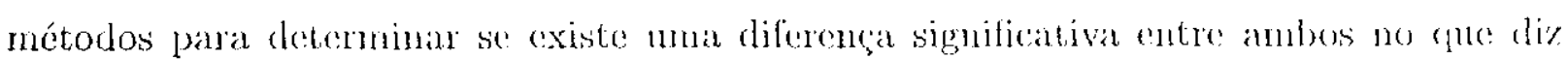

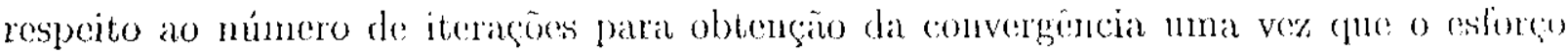

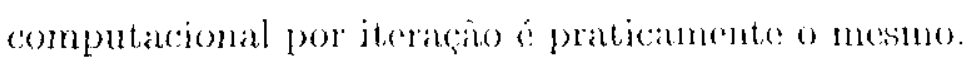


Com a climinaça de $y(4.5)$ se resume ao seguinte sistema näo-fincar:

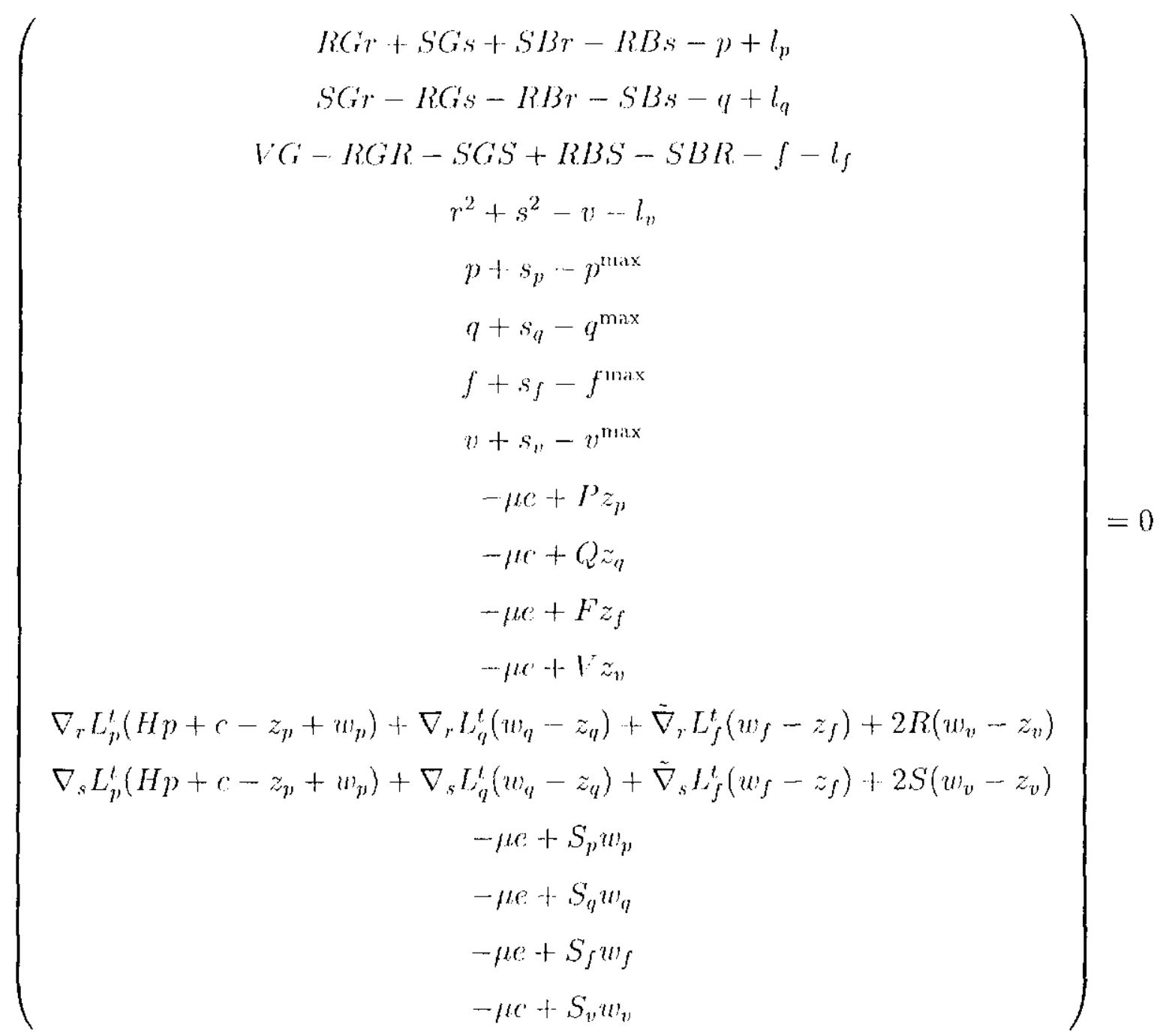

$\operatorname{com}(x, t) \geq 0$, onde:

$$
\begin{aligned}
& \tilde{\nabla}_{r} L_{f}^{\prime}=\nabla_{r} L_{f}^{\prime}+2 R G, \\
& \dot{\nabla}_{s} L_{f}^{t}=\nabla_{s} L_{f}^{t}+2 S G .
\end{aligned}
$$

\subsection{Método de Pontos Interiores Primal-Dual}

Dada uma classe de problenas, a forma padräo para desenvolver um método de pontos interiores consiste na aplicação do método de Newton [2] às condições de otimaliclade, desconsiderando as restrięoes de capacidade.

A convergencia do método a uma solıção é obtirla partindo-se de um ponto 


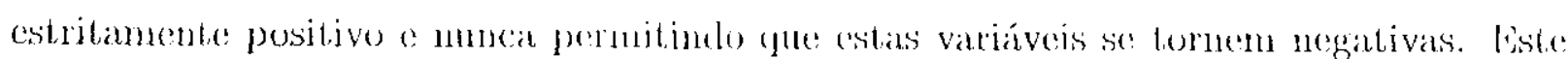
controle é readizado através do tamanho do passo. O métode resultante é essencialnente um método primal-dual esjecífico para esta classe de problemas [3].

O) método de pontos interiones primal-rlual para o problema (4.1) consiste portanto, na aplicacaío do método de Newton a (4.6) desconsiderando as restricions de não-negatividade $(x, t) \geq 0$. Fista eplicarăo resulta no seguinte método:

Método 4.1 (Método de Pontos Interiores)

Dados $\left(x^{0}, t^{0}\right)>0 e\left(r^{0}, s^{0}\right)$ livres.

Para $k:=0,1,2, \ldots, f a c a$ :

(1) Escolha $\beta^{k} \in(0,1)$ efaça $\mu^{k}=\beta^{k}\left(\frac{\gamma^{k}}{n}\right)$ onde, $\gamma^{k}=\left(x^{k}\right)^{l} t^{k}$ e n a a dimensüo do velor $x$.

(2) Calcule as direçoues de Newhon $\Delta x^{k}$ e $\Delta t^{k}$.

(3) Calcule o tamanho do passo para permanecer em um ponto interior

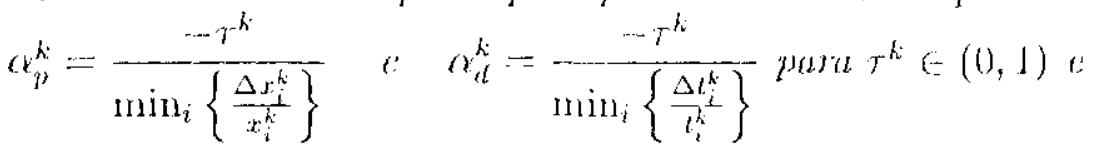

$$
\begin{aligned}
& \alpha^{k}=\min \left\{1, \alpha_{p}^{k}, \alpha_{i l}^{k}\right\} \text {. }
\end{aligned}
$$

(4) Cukluale o nowo ponlo

$$
\left(x^{k+1}, t^{k+1}\right)=\left(x^{k}, t^{k}\right)+e^{k}\left(\Delta x^{k}, \Delta l^{k}\right) .
$$

Os paranetros $\beta$ e $\tau$ e o ponto inicial serĩo discuticlos mais adiante. Deve-se adotar un tamanho de passo igual para as variáveis primaiss o duais devido à natureza quadrática do problema que como conseqüencia contém restriceocs onde aparecen variáveis primais e duais simultancamente. A escolha do valor para $\mu^{k}$ foi a princípio tomada bascando-se an programatsio linear, onde foram obtides excelentes resultados compulat cionais (ver Introdução), mas é interessante eseollier tek de forma dinâmica e apropriadia para cada tipo de problema. Temos como proposta jara un futuro trabalho, o estuclo desses parâmetros para este problema não-linear específico, objetivando a cliciência conputacional dos métodos implenentados (ver Capítulo 8). 


\subsubsection{Direções de Newton}

As direções de Newton são definidas pelo seguinte sistema linear ${ }^{1}$

$$
\begin{aligned}
& \left\{\begin{array}{l}
-\Delta p+\nabla_{r} L_{p} \Delta r+\nabla_{s} L_{p} \Delta s=r_{1} \\
-\Delta q+\nabla_{r} L_{q} \Delta r+\nabla_{s} L_{q} \Delta s=r_{2}
\end{array}\right. \\
& -\Delta f \text { । } G \Delta v+\nabla_{r} L_{f} \Delta r+\nabla_{s} L_{f} \Delta s=r_{3} \\
& -\Delta v+2 R \Delta r+2 S \Delta s=r_{1} \\
& \Delta p+\Delta s_{p}=r_{5} \\
& \Delta q+\Delta s_{q}=r_{6} \\
& \Delta f+\Delta s_{f}=r_{7} \\
& \Delta v+\Delta s_{n}=r_{8} \\
& Z_{p}, \Delta p+P \Delta z_{p}=r_{9} \\
& Z_{q q} \Delta q+Q \Delta z_{q q}=r_{10} \\
& Z_{j} \Delta f+H \Delta z_{f}=r_{1} \\
& Z_{n} \Delta v+V \Delta z_{n}=r_{12} \\
& \nabla_{r} L_{p}^{t}\left(I / \Delta p-\Delta z_{p}+\Delta w_{p}\right)+\nabla_{r} L_{l}^{\prime}\left(\Delta w_{q}-\Delta z_{q}\right)+\dot{\nabla}_{r} L_{f}^{\prime}\left(\Delta w_{f}-\Delta z_{f}\right)+ \\
& 2 R\left(\Delta w_{2},-\Delta z_{v}\right)+M \Delta r+N \Delta s=r_{13} \\
& \nabla_{s} L_{p}^{l}\left(H \Delta p-\Delta z_{p}+\Delta w_{p}\right)+\nabla_{s} L_{q}^{l}\left(\Delta w_{q}-\Delta z_{q}\right)+\tilde{\nabla}_{s} L_{f}^{\prime}\left(\Delta w_{f}-\Delta z_{f}\right)+ \\
& 2 S\left(\Delta w_{\eta},-\Delta z_{v}\right) \cdots N \Delta r+M \Delta s=r_{14} \\
& W_{p} \Delta s_{p}+S_{p} \Delta w_{p}=r_{15} \\
& W_{q} \Delta s_{q}+S_{q} \Delta w_{q}=r_{16 i} \\
& W_{S} \Delta s_{f}+S_{S} \Delta w_{S}=r_{1 T} \\
& W_{v} \Delta s_{v}+S_{v} \Delta w_{v}=r_{18}
\end{aligned}
$$

onde:

$$
\begin{aligned}
& M=G Y_{p}+Y_{p}\left(G-\left(B Y_{q}+Y_{q} B\right)+G Y_{f}^{\prime}+2 \zeta_{n},\right. \\
& N=B Y_{p} \cdots Y_{p} B+C Y_{q}-Y_{\eta} G+B Y_{f},
\end{aligned}
$$

e os resíduns de $r_{1}$ a $r_{18}$ são dados pela aplicação do ponto corrente $(x, t)$ ao lado esquerdo do sistema de equaçóes (4.6) com o sinal trocado.

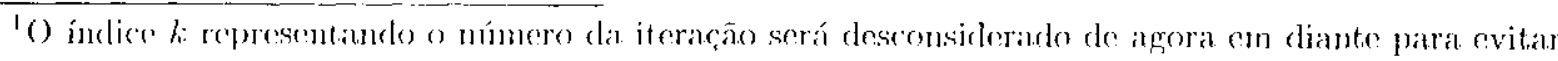
uma notação muito carrogada. 


\subsection{Método Preditor-Corretor}

() método preditor-cometor resolve dois sistemas lineares para encontrar as diregōes [13]. Primeimanente ó calcularda a direceäo afum $(\Delta \tilde{x}, \Delta \tau)$ resolvendo o sistenta linear (4.9) com $\mu=0$. En soguida, $\mu$ é caloulado e o seguinte sistema lincar re resolvido, obtendo-se a direção desejadia $(\Delta x, \Delta l)$ [11]:

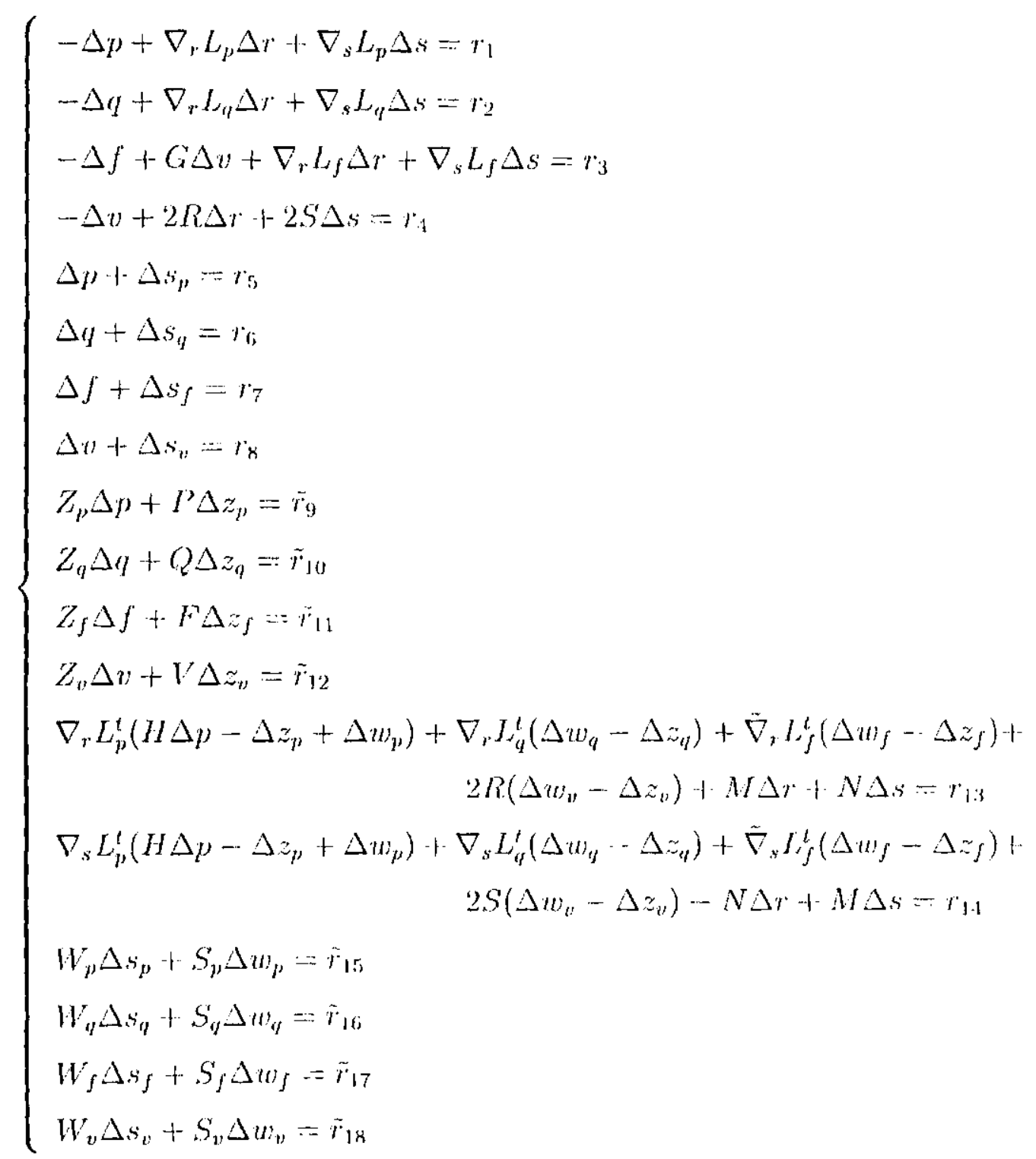

onde $\tilde{r}_{i}$ contém a soma entre o resíduo afim $r_{i}$, incluindo o valor calculado de $\mu$, a :

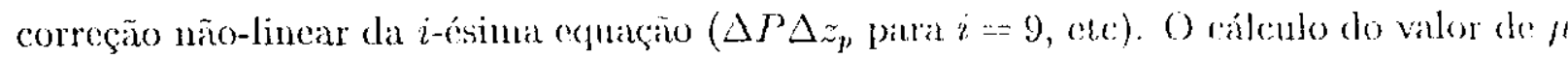
para o mótodo preditor-corretor será dedinido na proxima seçäo. 


\subsection{Detalhes de Implementação}

Nesta seção serão discutidos os detalhes de implementação dos métodos de pontos interiores desenvolvidos, com exceção da resolução do sistema linear que será discutida mais adiante. A implementação computacional desse trabalho foi feita para o método primal-dual aplicado ao problema relaxado, pois para o objetivo desse trabalho, não havia necessidade de restringir ofluxo de potência ativa nas linhas de transmissão. O acréscimo dessas restrições na implementação fazem parte de uma proposta futura (ver Capítılo 8).

\subsubsection{Considerações Iniciais}

Para a implementação foram utilizados alguns parâmetros e variáveis, como a precision, representada por e, o múmero de bantas de geraçäo $N G$, numero de barras com limites de geração de potencia reativa $N H$ e também representamos o número total de batrats por N/3

\subsubsection{Atualização das Variáveis}

As novas variaveis primais e duais sĩo calculadas da seguinte maneira:

$$
\begin{aligned}
& x^{k+1}=x^{k}+\alpha^{k} \Delta x \\
& t^{k+1}=t^{k}+\alpha^{k} \Delta t
\end{aligned}
$$

oncle o escalar ok $\alpha^{k} \in(0,1)$ co paranedro de comprimento do passo.

\subsubsection{Cálculo do Comprimento do Passo}

O comprimento máximo do passo $\alpha^{k}$ a determinado por:

$$
\begin{aligned}
& \alpha_{p}^{k \max }=\min _{\Delta x_{i}^{k}<0}\left\{\frac{-x_{i}^{k}}{\Delta x_{i}^{k}}\right\}, \\
& \alpha_{d}^{k^{\max }}=\min _{\Delta k_{2}^{k}<0}\left\{\frac{-l_{i}^{k}}{\Delta l_{i}^{k}}\right\}, \\
& \alpha^{k}=\min \left\{\tau \alpha_{p}^{k^{\max }} ; \tau \alpha_{d}^{k^{\max }} ; 1,0\right\} .
\end{aligned}
$$


() escalar $\tau \in(0,1)$ e um fator de seguranca para atsegenuar (que o próxino ponto satisfará as condiçós estritas de positividade. Um valor comum utilizado em programaça lineat

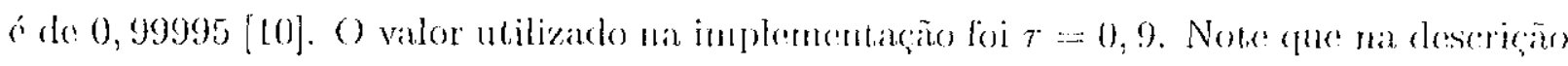

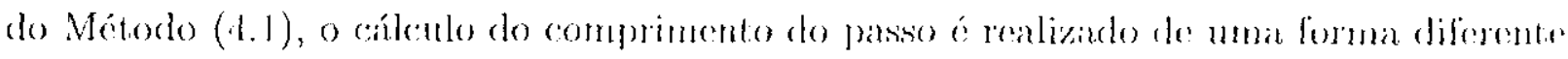
da apresentada nessa segão. A forma ntilizada no método é mma forma mais economita le se calcular o patsso, ambers levam ao mesmo resultarlo.

\subsubsection{Redução do Parâmetro de Barreira}

O residuo das condiçós de complenentaridade $\gamma$, chamado gap de complementaridade, na k-ésima itcraçào é:

$$
\gamma^{k}=\left(x^{k}+\Delta x^{k}\right)^{l}\left(t^{k}+\Delta t^{k}\right)
$$

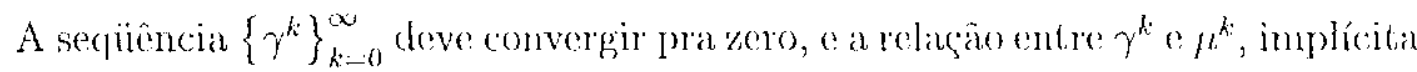
nas condiçoes de KK'l, sugerem que $\mu^{k}$ poeleria ser reduzids baseado muma diminuição prevista do gap de complementaridade, como:

$$
\mu^{k+1}=\beta^{k} \frac{\gamma^{k}}{2 \cdot(N G+N H)} .
$$

() parametro $\beta^{k} \in(0,1)$ é chanado de parametros de centralizaçäos é interpre-

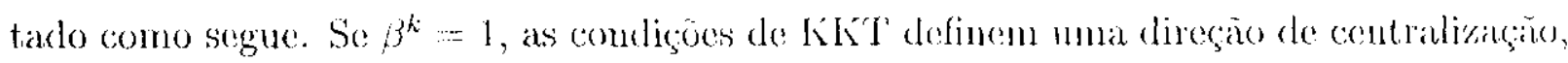
um passo de Newton em direcão ao ponto na trajetória da barreira. Por ontero lacko, $\beta^{k}=0$ temos o passo de Newton puro, conhecido conno direção afint-escala. Para reduçano do $\mu^{k}$ melhorar a centratizargão, $\beta^{k}$ a dinamicamente escollido como $\beta^{k}=\max \left\{0,95 / \beta^{k-1} ; 0,1\right\}$, (o) $\beta^{0}=0,2$

\subsubsection{Critério de Convergência}

As iteracoeses do mólodo são consideradas terminiadas culando:

$$
\operatorname{sro}=\operatorname{milx}\left\{v_{n}^{k}, \hat{\gamma}^{k}\right\} \leq c
$$


sendo $\nu_{n}^{k}$ em função das normas relativas dos resíluos e $\hat{\gamma}^{k}$ o gall relativo à função objetivo, que são calculados da seguinte mancira:

$$
\begin{aligned}
& \nu_{n}^{k}=\max \left\{\frac{\left\|r \eta^{k}\right\|}{n p^{k}}, \frac{\left\|r q^{k}\right\|}{n q^{k}}, \frac{\left\|r v^{k}\right\|}{n w}, \frac{\left\|r s \eta^{k}\right\|}{n p u}, \frac{\left\|r s q^{k}\right\|}{n q u}, \frac{\left\|r s v^{k}\right\|}{n w u}, \frac{\left\|r r^{k}\right\|}{n c}, \frac{\left\|r s^{k}\right\|}{n c}\right\} \\
& \gamma^{k}-|-| \frac{\gamma^{k}}{1+\left|2 \cdot \phi\left(p^{k}\right)\right|} \cdot \frac{1}{n n n} \mid
\end{aligned}
$$

onde:

$$
\begin{aligned}
& \epsilon=10^{-3}, \\
& n p^{k}=1+\left\|p^{k}\right\|+\left\|r^{k}\right\|+\left\|\cdot s^{k}\right\|, \\
& n q^{k}=1+\left\|q^{k}\right\|+\left\|r^{k}\right\|+\left\|s^{k}\right\|, \\
& m u=1+\left\|v^{\min ^{2}}\right\|, \\
& n m u=1+\left\|p^{\max }\right\|, \\
& n q u=1+\left\|q^{\max }\right\|, \\
& n m u=1+\left\|v^{\max }\right\|, \\
& n c=1+\|\|+c \cdot \|, \\
& n n n=2 \cdot(N G+N I+N B),
\end{aligned}
$$

o os resíduos:

$$
\begin{aligned}
& r p=p+l_{p}-R C r-S G s-S B r+R B s \\
& r q=q+l_{q}-S C W+R C s+R B r+S B s, \\
& r v=v^{\text {minn }}{ }^{2}-r^{2} \cdots s^{2}+0, \\
& r s p=p^{\max }-p-s_{p}, \\
& r s q q: q^{\max } \cdot \eta \cdots s_{q} \\
& r s v=v^{\text {minax }}-v-s, \\
& r r=-2 R y_{v}-\nabla_{r} L_{p}^{\prime} y_{p}-\nabla_{r} L_{q}^{\prime} y_{q}, \\
& r s=-2 S y_{v}-\nabla_{s} L_{p}^{\prime} y_{p}-\nabla_{s} L_{q}^{\prime} y_{q} .
\end{aligned}
$$




\subsubsection{Ponto Inicial}

O ponto inicial apresentado a seguir a o mesmo utilizado no nodelo DC: [18], arereiclas as variáveis relacionaldas a modelo reativo:

$$
\begin{aligned}
& p-s_{p}=-p_{2}^{m a x}, \quad \quad z_{p}, \cdots, w_{p}, \cdots=c^{\prime}, \\
& q \because s_{q}=\frac{q_{q}^{\prime m+1}}{2 m}, \quad \quad z_{q} \cdot w_{q}=: e \text {, } \\
& f=-s_{f}=f_{z}^{m a x}, \quad \quad z_{j}-w_{f}=t, \\
& y=s_{v}-u^{m a x}, \quad z_{v}=w_{v}=e \text {, } \\
& r=s \cdot \sqrt{\eta} \cdot
\end{aligned}
$$

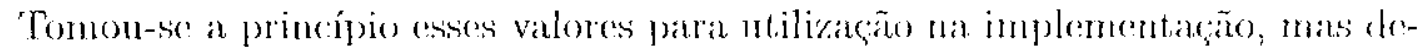
vido ao resultado insatisfiatório das variaveis nas iterações a a não convergencia do método, procurou-so outra forma para determinal pontos iniciais.

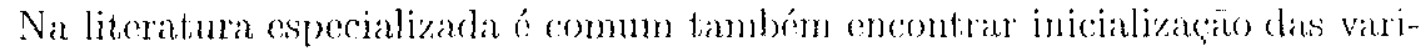
áveis com valores unitários, o que também foi realizado na implementação desse traballı, obtendo-se resultados melhores que os anteriores mas ainda assim o medodo näo convergiu, devido à natureza altamente nào-linear do problema.

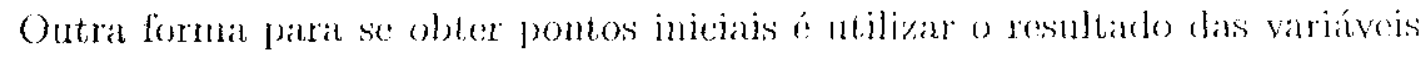
do problema de fluxo de carga, gue geralmente fornece bons iudicadores do restido des sistema. 


\section{Capítulo 5}

\section{Resolução do Sistema Linear}

A matriz dos sistemas lineares dos métodos primal-dual e preditor-corretor é a mesna. Portando a discussä́o desta sẹão se restringirá ao sistema (1.9). Este sistena linear pode ser resolvido diretannente. No entanto á mais vantajoso redurir a dimensão do sistema através da climinaçấo dass variáveis de folga sem modificar sua estrutura cepparsa [23]. Primeiranente substituínos as variáveis de folgin mimais:

$$
\begin{aligned}
& \Delta s_{p_{1}}=r_{5}-\Delta p, \\
& \Delta s_{s_{1}}=r_{6}-\Delta q, \\
& \Delta s_{f}=r_{7}-\Delta f, \\
& \Delta s_{s_{1}}=r_{8}-\Delta y,
\end{aligned}
$$




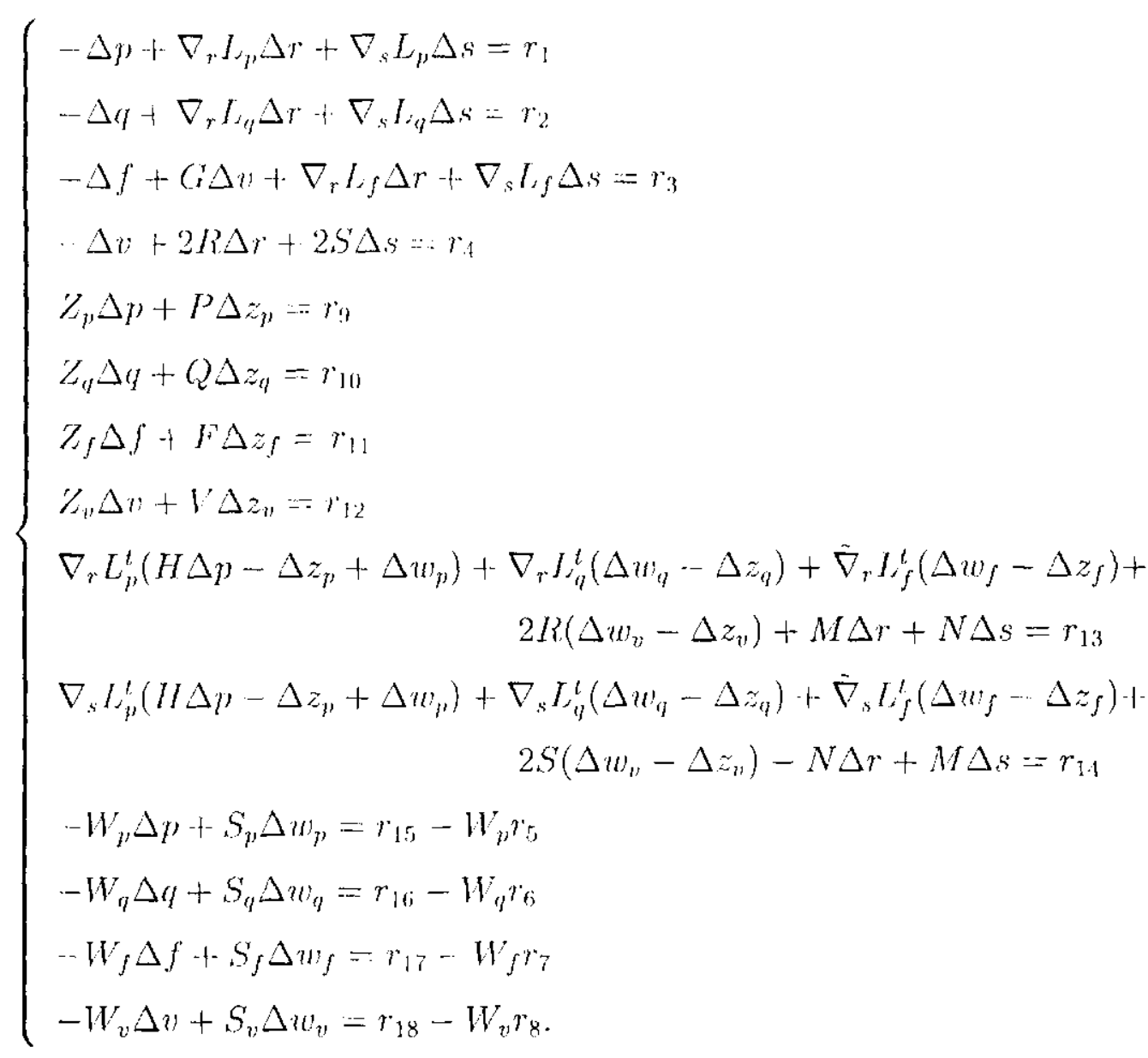

Fliminando agora as variáveis de folga duais:

$$
\begin{aligned}
& \Delta z_{p}=P^{-1}\left(r_{9}-Z_{p} \Delta p\right), \\
& \Delta z_{q}=Q^{-1}\left(r_{10}-Z_{q} \Delta q\right), \\
& \Delta z_{f}=F^{-1}\left(r_{11}-Z_{f} \Delta f\right), \\
& \Delta z_{\eta}=V^{1}\left(r_{12}-Z_{\eta} \Delta v\right), \\
& \Delta w_{\eta}=S_{p}^{-1}\left(r_{15}+W_{p}\left(\Delta p-\cdots r_{5}\right)\right), \\
& \Delta w_{q}=S_{q}^{-1}\left(r_{16}+W_{q}\left(\Delta q-r_{6}\right)\right), \\
& \Delta w_{f}=S_{j}^{1}\left(r_{17}+W_{f}\left(\Delta f-r_{7}\right)\right), \\
& \Delta w_{\eta}=S_{v}^{1}\left(r_{18}+W_{v}\left(\Delta v-r_{8}\right)\right),
\end{aligned}
$$


o sistema linear se relat at

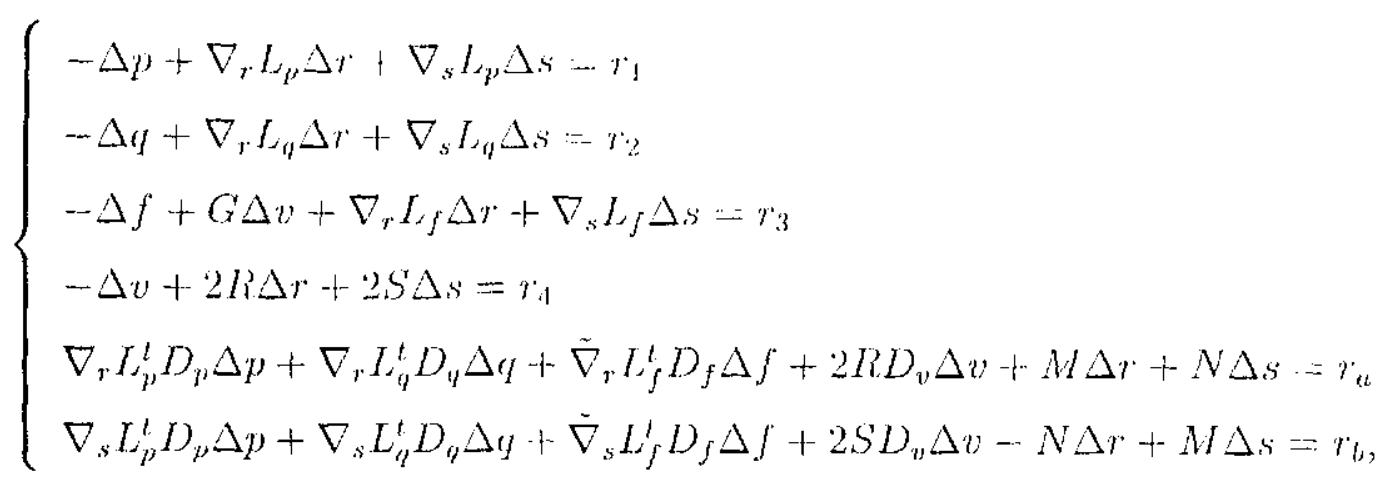

onde:

$$
\begin{aligned}
& D_{p}=P^{1} Z_{p}+S_{p}^{-1} W_{p}+H, \\
& D_{q}=Q^{-1} / z_{q u}+S_{q}^{-1} W_{q}, \\
& D_{f}=r \cdot \cdots \cdot z_{f}+S_{f}^{-1} W_{f}, \\
& D_{v}=V^{-1} z_{v}+S_{v}{ }^{\prime} W_{v},
\end{aligned}
$$

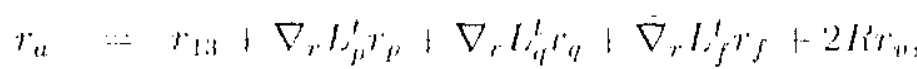

$$
\begin{aligned}
& r_{b}=r_{14}+\nabla_{s} L_{p}^{t} r_{p}+\nabla_{s} L_{q}^{\ell} r_{q}+\dot{\nabla}_{s} L_{f}^{t} r_{f}+2 S_{r_{v}} \\
& r_{p}=P^{\cdots r_{9}}+S_{p}^{-1}\left(W_{p} r_{5}-r_{15}\right), \\
& r_{q}=Q^{-1} r_{10}+S_{q}^{r^{1}}\left(W_{q} r_{6}-r_{16}\right) \text {, } \\
& r_{f}=F^{2} r_{11}+S_{f}{ }^{\prime}\left(W_{f} r_{f}-r_{17}\right) \text {, } \\
& r_{y}=V^{-1} r_{12}+S_{v}^{-1}\left(W_{v} r_{8}-r_{18}\right) \text {. }
\end{aligned}
$$

Somente inverwas de matrizes diagonass são envolvidas nestas substituiçöes. As substituiçơses de variáveis encontradas na literatura terminann neste pontes o o epuivalente ao sistema (5.1) é resolvido. No entanto, a eliminação das variáveis de geração ativa o reativa, fluxo de potoncia e temsios:

$$
\begin{aligned}
& \Delta p=\nabla_{r} L_{y} \Delta r+\nabla_{s} L_{j p} \Delta s-r_{1}, \\
& \Delta q=\nabla_{r} L_{q} \Delta r+\nabla_{s} L_{q} \Delta s-r_{2}, \\
& \Delta f=\left(i \Delta v+\nabla_{r} L_{f} \Delta r+\nabla_{s} L_{j} \Delta s-r_{3},\right. \\
& \Delta v=2 R \Delta r+2 S \Delta s-r_{1},
\end{aligned}
$$

resulta em:

$$
\left\{\begin{array}{l}
A_{r r} \Delta r+A_{r s} \Delta s=\pi_{a} \\
A_{s r} \Delta r+A_{s s} \Delta s=T_{b},
\end{array}\right.
$$


onde:

$$
\begin{aligned}
& A_{r r}=M+\nabla_{r} L_{p}^{t} D_{p} \nabla_{r} L_{p}+\nabla_{r} L_{q}^{\prime} D_{q} \nabla_{r} L_{q q}+\check{\nabla}_{r} L_{f}^{l} D_{f}\left(\nabla_{r} L_{f}+2 G R\right)+4 R D_{v} R, \\
& A_{r s}=N+\nabla_{r} L_{p}^{t} D_{p} \nabla_{s} L_{p}+\nabla_{r} L_{q}^{t} D_{q} \nabla_{s} L_{q}+\tilde{\nabla}_{r} L_{f}^{t} D_{f}\left(\nabla_{s} L_{f}+2 G S\right)+4 R D_{\eta} S \text {, } \\
& A_{s r}=-N+\nabla_{s} L_{p}^{t} D_{p}, \nabla_{r} L_{p}+\nabla_{s} L_{i q}^{t} D_{q} \nabla_{r} L_{q q}+\dot{\nabla}_{s} L_{f}^{t} D_{f}\left(\nabla_{r} L_{f}+2 G R\right)+4 S D_{v} R, \\
& A_{s s}=M+\nabla_{s} L_{p}^{t} D_{p} \nabla_{s} L_{p}+\nabla_{s} L_{q}^{t} D_{q} \nabla_{s} L_{q}+\dot{\nabla}_{s} L_{f}^{t} D_{f}\left(\nabla_{s} L_{f}+2 G S\right)+4 S D_{v} S \text {, } \\
& i_{n}=r_{a}+\nabla_{r} L_{p}^{\prime} D_{p} r_{1}+\nabla_{r} L_{q}^{\prime} D_{q} r_{2}+\dot{\nabla}_{r} L_{f}^{\prime} D_{f}\left(r_{3}+C r_{4}\right)+2 R D_{r} r_{4}, \\
& \hat{r}_{b}=r_{b}+\nabla_{s} L_{p}^{t} D_{p} r_{1}+\nabla_{s} L_{i q}^{t} D_{q} r_{2}+\tilde{\nabla}_{s} L_{f}^{t} D_{f}\left(r_{3}+G r_{4}\right)+2 S D_{l} r_{4} \text {. }
\end{aligned}
$$

Devido à simetria das matrizes $G$ e $B$ (ver Seção 2.3):

$$
\begin{aligned}
& \nabla_{r} L_{f}+2 G R=\tilde{\nabla}_{r} L_{f}, \\
& \nabla_{s} L_{f}+2 G S=\tilde{\nabla}_{s} L_{f},
\end{aligned}
$$

substituímos (5.5) em (5.4) temos:

$$
\begin{aligned}
& A_{r r}=M+\nabla_{r} L_{p}^{t} D_{p} \nabla_{r} L_{p}+\nabla_{r} L_{q}^{t} D_{q} \nabla_{r} L_{q}+\tilde{\nabla}_{r} L_{f}^{t} D_{f} \dot{\nabla}_{r} L_{f}+4 R D_{v} R, \\
& A_{r s}=N+\nabla_{r} L_{p}^{t} D_{p} \nabla_{s} L_{p}+\nabla_{r} L_{q}^{t} D_{q} \nabla_{s} L_{q}+\dot{\nabla}_{r} L_{f}^{t} D_{f} \dot{\nabla}_{s} L_{f}+4 R D_{v} S, \\
& A_{s r}=-N+\nabla_{s} L_{p}^{t} D_{p} \nabla_{r} L_{p}+\nabla_{s} L_{q}^{t} D_{q} \nabla_{r} L_{q}+\tilde{\nabla}_{s} L_{f}^{t} D_{f} \dot{\nabla}_{r} L_{f}+4 S D_{v} R, \\
& A_{s s}=M+\nabla_{s} L_{p}^{t} D_{p} \nabla_{s} L_{p}+\nabla_{s} L_{q}^{t} D_{q} \nabla_{s} L_{q}+\tilde{\nabla}_{s} L_{f}^{t} D_{f} \tilde{\nabla}_{s} L_{f}+4 S D_{v} S, \\
& \tilde{r}_{a}=r_{r}+\nabla_{r} L_{p}^{t} D_{p} r_{1}+\nabla_{r} L_{q q}^{t} D_{q} r_{2}+\tilde{\nabla}_{r} L_{f}^{t} D_{f}\left(r_{3}+G r_{1}\right)+2 R D_{v} r_{4}, \\
& \tilde{r}_{b}=r_{b}+\nabla_{s} L_{p}^{t} D_{p} r_{1}+\nabla_{s} L_{q}^{t} D_{q} r_{2}+\dot{\nabla}_{s} L_{f}^{t} D_{f}\left(r_{3}+G r_{4}\right)+2 S D_{v} r_{4} .
\end{aligned}
$$

Estas últimas substituições (5.2) alteram a estrutura matricial do sistema de forma mais radical. A esparsidarle dos blocos matriciais envolvidos é, até antes desta última transformação (5.3), determinada pelas linhas da rede de transmissão onde cada linlıa corresponde a um elemento não nulo nas matrizes envolvidas. Una vez que os novos blocos matriciais são formados por produtos dos blocos anteriores, oles correspondem a uma rede onde novas linhas surgen entre barras que se encontram a uma distancia de comprimento dlois na rede original.

Essa abordagem parece ser indicada para sistemas reais, pois o enchimento da matriz ć relativamente pecfueno e como veremos a segulir, a matriz do sistema linear (5.3) tem propriedades interessantes. 


\subsection{Estrutura Matricial}

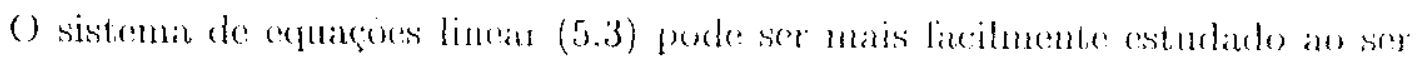
escrito da sogrinte fomat:

$$
\begin{aligned}
& {\left[\left(\begin{array}{cc}
M & N \\
-N & M
\end{array}\right)+\left(\begin{array}{cc}
\nabla_{r} L_{p}^{l} & 0 \\
0 & \nabla_{s} L_{p}^{\prime}
\end{array}\right)\left(\begin{array}{cc}
D_{p} & D_{p} \\
D_{p} & D_{p}
\end{array}\right)\left(\begin{array}{cc}
\nabla_{r} L_{p} & 0 \\
0 & \nabla_{s} L_{p}
\end{array}\right)+\right.} \\
& \left(\begin{array}{cc}
\nabla_{r} L_{q}^{t} & 0 \\
0 & \nabla_{s} L_{q}^{t}
\end{array}\right)\left(\begin{array}{cc}
D_{q} & D_{q} \\
D_{q} & D_{q}
\end{array}\right)\left(\begin{array}{cc}
\nabla_{r} L_{4} & 0 \\
0 & \nabla_{s} L_{\iota^{\prime}}
\end{array}\right)+ \\
& \left(\begin{array}{cc}
\dot{\nabla}_{r} L_{j}^{l} & 0 \\
0 & \dot{\nabla}_{s} L_{f}^{l}
\end{array}\right)\left(\begin{array}{cc}
D_{j} & D_{j} \\
D_{j} & D_{j}
\end{array}\right)\left(\begin{array}{cc}
\tilde{\nabla}_{r} L_{f} & 0 \\
0 & \tilde{\nabla}_{s} L_{f}
\end{array}\right)+ \\
& \left.4\left(\begin{array}{cc}
R & 0 \\
0 & S
\end{array}\right)\left(\begin{array}{cc}
D_{w} & D_{w} \\
D_{v} & D_{w}
\end{array}\right)\left(\begin{array}{cc}
R & 0 \\
0 & S
\end{array}\right)\right]\left(\begin{array}{c}
\Delta r \\
\Delta s
\end{array}\right)=\left(\begin{array}{l}
\tilde{r}_{n} \\
\tilde{r}_{b}
\end{array}\right) .
\end{aligned}
$$

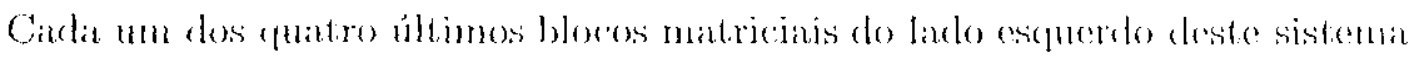

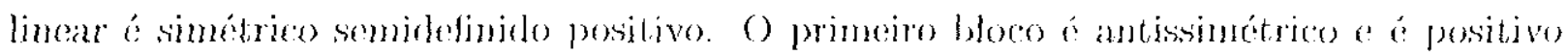
definido, so a somente so, $M$ ó uma matriz defonida positiva pois [6]:

$$
\left(\begin{array}{ll}
a & b
\end{array}\right)^{\prime}\left(\begin{array}{cc}
M & N \\
-N & M
\end{array}\right)\left(\begin{array}{l}
a \\
b
\end{array}\right)=a^{\prime} M a+b^{\prime} M b
$$

para quaisquer votores $a$ o b de dimensato apropulada.

Não existe garantia que a matriz $M$ seja definida positiva mas por ontro lacdo a existencia de cuatro blocos matriciais semidefinidos positivos indica que esta matriz deve permanecer numericamente estável ao longo das iteracoeses dos métodos de puntos interiores en comparação com a matriz do sistena linear (5.1). Além disso, embora nào

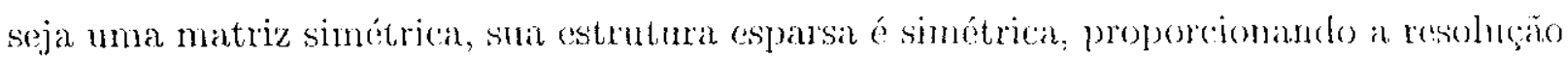
do sistema linear de forma mais eficiente que para matrizes assimétricas en geral. 


\section{Capítulo 6}

\section{Resultados Computacionais}

\subsection{Implementação e Ambiente}

A implementação desse projeto foi feita inicialmente em MatLaB versão 5.3.0 (R11), pois permitiu facilidade de manipulação con as malrizes e variáveis, sem preocupagão con reserva e enderegamente de memória, entre outras vantagens. Mats a grande desvantagem dessa implementaçõo em MÁtLAB for que ela não exploron a c'strutura esparsa desses problemas. Devido principalmente à esse fato e também o alto gran de não-linearidade, ocorreram unutos problemas para a convergência do prograna. Precisou-se encomtrar ajustes muito fimos nos parâmetros para que as condiçóces de otimalidade e complementaridade do problema fossem satisfeitas, fazendo com que os resíduos primais e duais estivessem dentro de um limite com uma precisão consideravelmente satisfatória. Essa busca por parâmetros fora feita combinando pontes iniciais, valores iniciais para o paràmetro de barreira, parámetro de centralização e até mesmo no cálculo do passo, através de mudança no valor do fator de segurança. Realizon-se extensivos testes computacionais, utilizando várias combinasoues de parâmetros para poder definir quais seriàm utilizadas, e quais eram as mohores escolhas para cadla sistema.

Os testes foran realizados em un computador com processador i686, clock de 1700GI\%, memória RA.l de 512MB, utilizando o sistema operacional FrenBSD-Unix.

Todos os resultades o valores ntilizados tanto para realizanono dos testes, como para a apresentação, săo dados rom pu, exceto algumas variáveis que näo estäo convertidas 
para a base do sistema em questão, nesse caso será feita a declaração da grandezas das mesmas.

\subsection{Sistemas}

Foram realizados testes com vários sistemas, com características e estruturas diferentes. Apresentaremos nesse capítulo os resultados computacionais extraíclos de testes em dois sistemas:

- BAR3 - 3 barras e 3 ramos;

- IEEE30 - 30 barras e 41 ramos.

O sistema BAR3.3 o um sistema de testes que foi criado exchusivamente para obter melhores ajustes no programa, pois o ponto inicial utilizarlo foi proveniente do resiltado do problema de fluxo de carga, o due geralmente é un ponto razóncl. Como consegüencia, conseguin-se estudar methores alternativas para os valores iniciais dos parâuetros c suas atualizaçŏcs.

O sistema IEEE30 ó um problema já conhecido e vtilizado por pescquisadores da área de sistemas de potencia para testes computacionais. Bncontra-se experimentos e resultados utilizando cese sistema em grande parte de documentos técuicos e artigos em revistas especializadias.

Para a realização dos testes utilizon-se escunemas com valores diferentes para os limitantes de tensão nas barras, pois dessa forma poderia-se analisar com mais detalhes o conportanento do sistema sob um forte controle e restriçĩo, e isso pode ser realizado devido a uma boa cestrutura do modelo. Os escuemas utilizados sĩo mostrarlos na Tabela 6.1.

Tabela 6.1: Limites de tensäo

\begin{tabular}{c|c|c|c|c|c}
\hline limite & L-A & L-B & L-C & L-D & L-E $^{*}$ \\
\hline inferior & 0,90 & 0,94 & 0,98 & 0,99999 & 0,97 \\
superior & 1,10 & 1,06 & 1,02 & 1,00001 & 1,03 \\
\hline
\end{tabular}




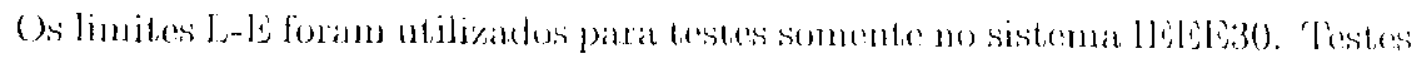

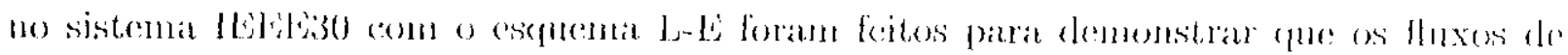
potencia ativa nas linhas portem ser controlados limitando a geragâa de potencia ativa

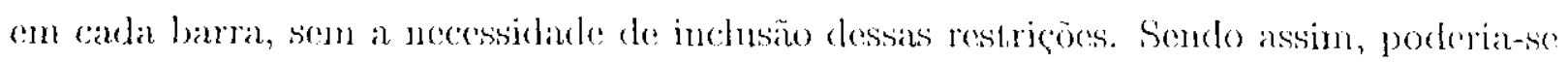
gerar uma implementacão baseada no modelo para o problema relaxado (3.2), palua josos)

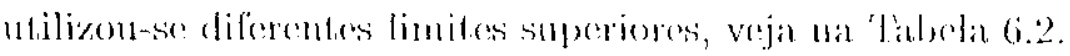

Tabela 6.2: Custos e limites de gerução

\begin{tabular}{c|c|c}
\hline sistema & $\left.\frac{1}{2} I\right]$ & $y^{\text {rniax }}$ \\
\hline BAR3 & 0,5 & 1,0 \\
IEEE30 & 0,5 & $0,501,0$ \\
\hline
\end{tabular}

Utiliza-so una unidade moneturia inaghinária, sem atribuiçono de moeda. Considera-se o linite $p^{\text {max }}$ para cada barra geradora de potencia ativa. Foram feitos testes em dois valores para $p^{\text {max }}$ no sistema IEEE30 acima apresentarlos. O objetivo desses trestes foi verificar mudanças nos valores do fluxos de potência ativa nas linhas de transmissão e quanto seriam representativas essals alteraçoes. Nas seçoes seguntes serão feitas análises que methor demonstram esses testes.

Os resultados dos testes realizados nos sistemas BAR3 e IELE30 säo apresentados nas seçôs seguintes atraves de tabelas, onde pode-se realizalr mna comparaçion dos valores obtidos para os esquemas utilizados, como o número de iteracoes para a convergência do métudo, o valor da funçăo objetivo o o $\hat{\gamma}$ - gap de complementaridaule relativo à esse valor, tempo total giasto e numero de operaçöes de ponto flut uante realizadas durante a execuçäo (llops).

\subsection{BAR3}

O sistema BAR3 é consideravelmente pequeno enn relação à outros sistculas de testes. A resolução desse sistena serviu cono referencia em relaça à implementaçio do método para comparações futuras conno o sistema IEEE30, pois possui um bom comportamento e seus parámetros foran consideravelmente mitis simples de definir. Outro fator 
importante foi o ponto inicial, obtido através da resolução do problema de fluxo de carga, o que nos permite um bom ponto de operação inicial para o sistema. Primeiramente utilizado, o sistema BAR3 fornecen dados importintes para mua avaliarano do método implementado com a dedução matemática do modelo. Nesse momento criou-se também en paralelo uma implementação en linguagen C com apenas alguns módulos, principalmente o incial, para efejto comparativo com a implementação existente en termos do cstrutura e resultados, a fim de procurar um melhor ajuste.

Nas seçós seguintes serão apresentados os resultados dos valores para tensĩo, geraçăo de potencia ativa, função objetivo e a noma resirlual relativa ì esso valor, fluxos a perdas de potêneja ativa nas linhas de transmissão. Analison-se o sistema BAR3 com a aplicação de todos os esquemas. Graficamente priorizon-se o esquema L-D, onde foi possiver unn controle matior sobre as tensores.

\subsubsection{Análises}

O programa convergiu para todos os escememas, o que de cecta forma permite un controle desejável para a tensão nas barras. Nota-se também que o tempo computacional para a resolução desse sistema foi muito pequeno. Estão tambén dispostos na Tabela 6.3 o mumero de iteraçóes necessárias e o número de operaçôes realizadas para a convergência do método, onde o c nuilizado foi $10^{-3}$.

\begin{tabular}{|c|c|c|c|c|}
\hline esquema & iterações & $\phi$ & tempo $[s]$ & flops \\
\hline$L-A$ & 5 & 0,379 & 0,015 & 14053 \\
\hline $1,-13$ & 5 & 0,379 & 0,015 & 1405.3 \\
\hline $\mathrm{L}-\mathrm{C}$ & 6 & 0,379 & 0,015 & 16812 \\
\hline$(,-I)$ & 6 & 0,379 & 0,016 & 16812 \\
\hline
\end{tabular}

Nos escquemas $L-A, I,-B$ o $L_{-}$- $:$, o valor do erro é dado pelo valor do $\hat{\gamma}$, pois

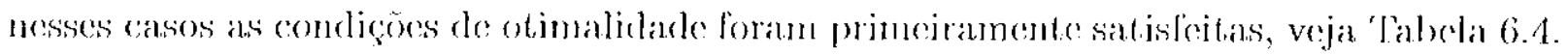
A partir da restrição máxima nos limites da tensũo, no esquema 1 -D), pode-se observar 


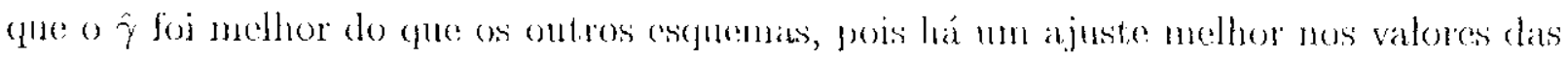

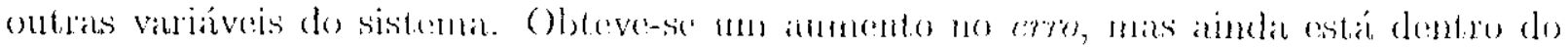

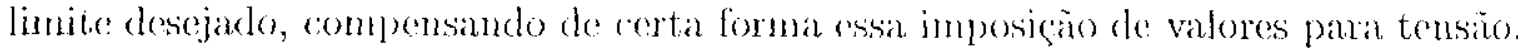

Tabela 6.4: Valor do erTo equy de complementaridude relation BARS

\begin{tabular}{|c|c|c|}
\hline esquema & $\cos c$ & $\ddot{\gamma}$ \\
\hline $1-A$ & $0,66 j 50 \times 10 \mathrm{c}-3$ & $0,6650 \times 100-3$ \\
\hline $\mathrm{I}_{2}-\mathrm{B}$ & $0,7115 \times 10 \mathrm{c}-3$ & $0,7115 \times 10 \mathrm{c}-3$ \\
\hline L-C & $0,3155 \times 1000-3$ & $0,3155 \times 10(0,-3$ \\
\hline $1-1)$ & $0,7188 \times 100-3$ & $0,2393 \times 10 c-3$ \\
\hline
\end{tabular}

\subsubsection{Tensão}

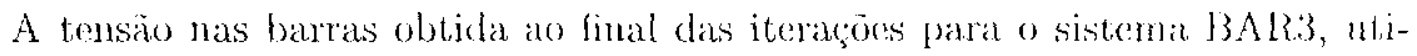

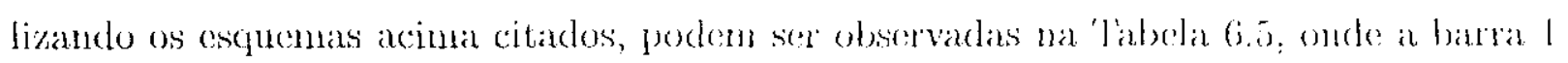
Ea barra de referencia do sistonia.

\begin{tabular}{|c|c|c|c|c|}
\hline barra & L-A & L-B & $\mathrm{L}-\mathrm{C}$ & L-D \\
\hline $1^{*}$ & 1,015 & 1,0099 & 1,005 & 1,0000 \\
\hline 2 & $1,01: 2$ & 1,005 & 1,002 & 1,0000 \\
\hline 3 & 1,001 & 0,995 & $(0,095)$ & $1,(0)(0)$ \\
\hline
\end{tabular}

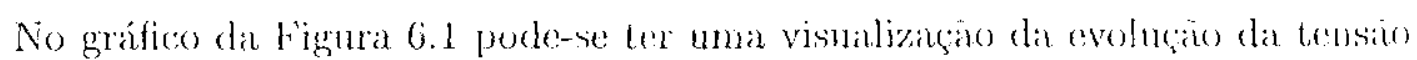

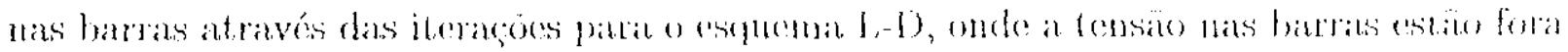
dos limites no ponto inicial o säo forchalas a 1,0 , 3 u.

\subsubsection{Potência Ativa}

A garação de potencia ativa é leita semente nas barrass de geraçio do sistema.

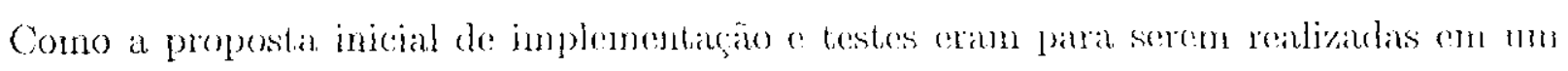




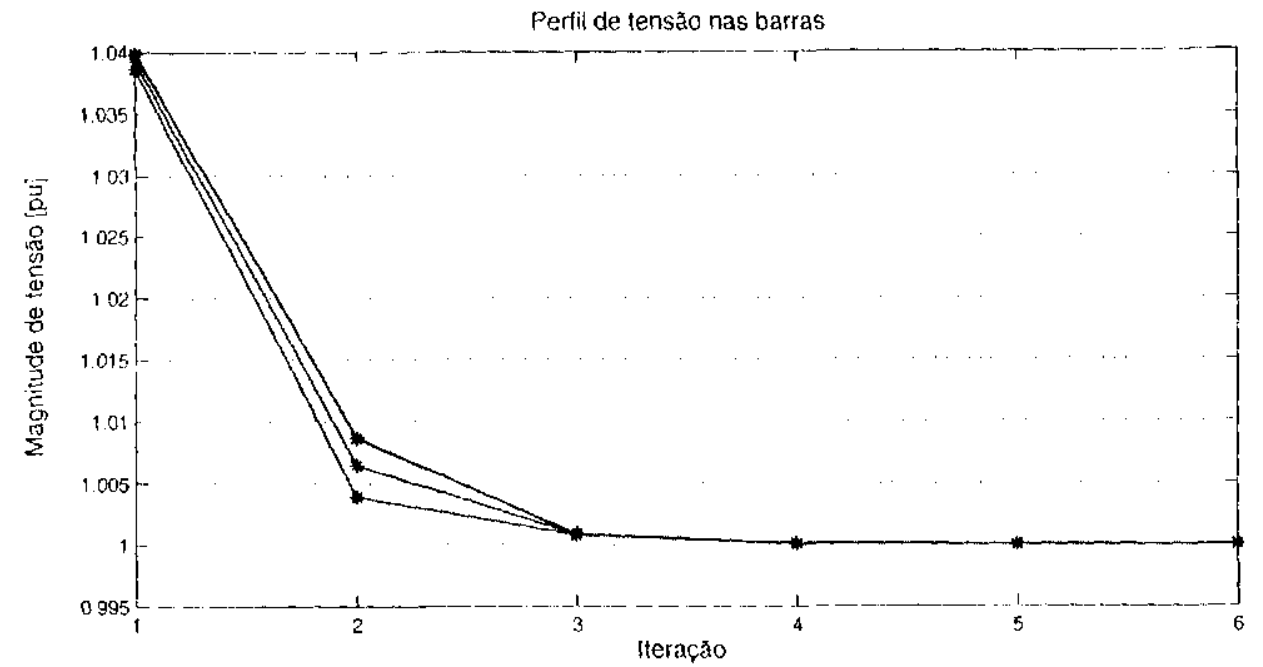

Figura 6.1: Tensão nas barras - BAR3 $(I,-D)$

sistema sinples, então estabelecen-se que no sistema BAR3, todas as barras poderiam gerar potencia ativa. Os resultados linais das itoracos podem ser vistos na Tabela 6.6 .

Tabcla 6.6: Goraçá de polência ativa $B$ BA
\begin{tabular}{c|c|c|c|c}
\hline barras & L-A & L-B & L-C & L-D \\
\hline 1 & 0,501 & 0,501 & 0,501 & 0,498 \\
2 & 0,512 & 0,513 & 0,512 & 0,518 \\
3 & 0,494 & 0,494 & 0,495 & 0,491 \\
\hline
\end{tabular}

A geraç̃o de potência nas barras para o escuema $L_{-}-\mathrm{D}$ estaño mostradas no gxálico da lïgura 6.2, onde as barras inicialmente possuem valores de potência baixos, pois o problema de fluxo de carga não considema as condiçôes de otimalidade. Ao longo das iteraçoos esses valores são ajustados em conjunto com as outras variáveis para que as condições de otimalidade o complementaridade sejam atendidas, mantendo também os pontes interiores. 


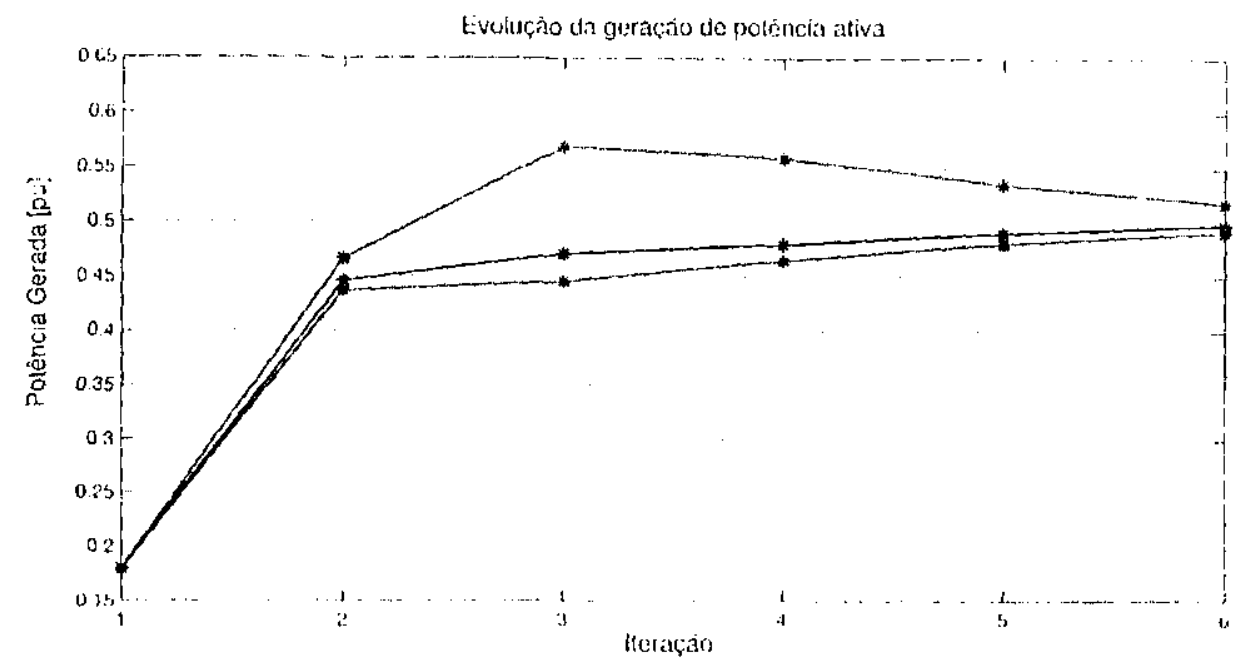

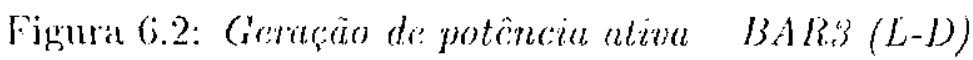

\subsubsection{Normas Residuais Relativas}

As normas residuais relativas à função objetivo são utilizadas para testar a convergência do métorlo, são também indicadores de desempenho da implementação. Utilizise esses valores para detectar e indicar possíveis direcooses emarlas, pontos iniciais ruins ou até mosmo parânetros näo apropriados para o sistema. No grálico da Fignta 6.3 pode-

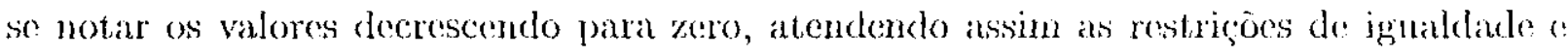
desigualdades do problema (3.2).

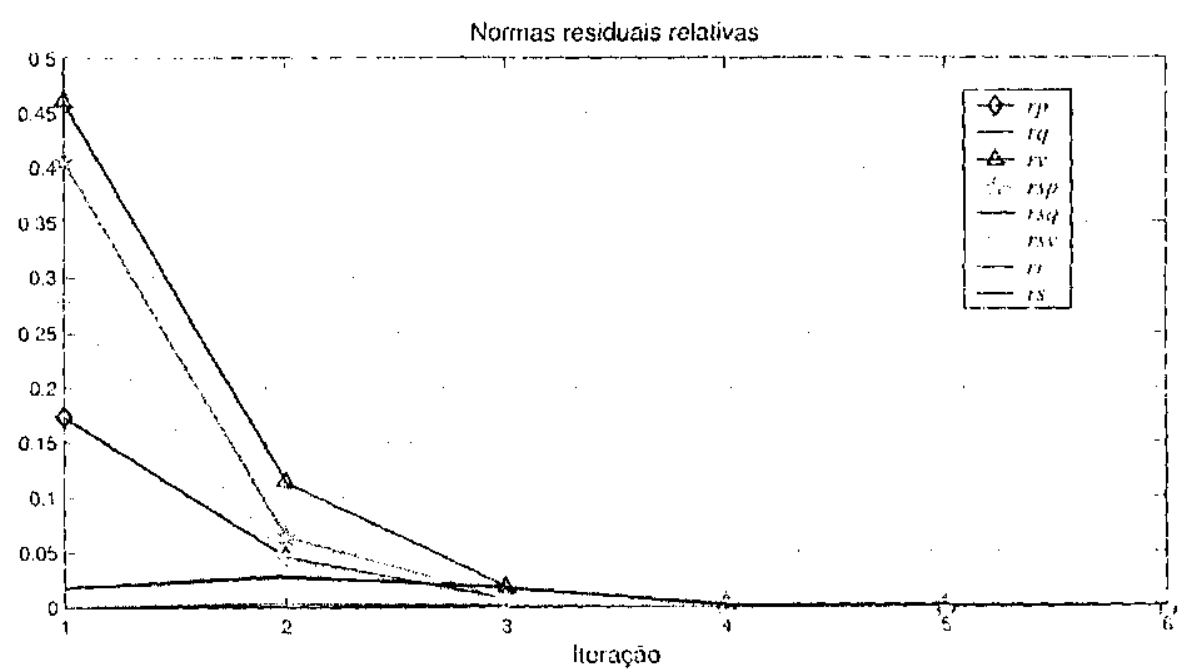

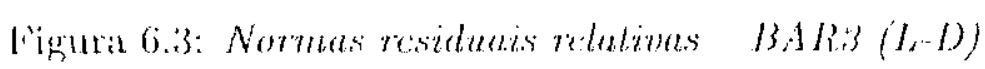




\subsubsection{Fluxo e Perda de Potência Ativa nas Linhas de}

\section{Transmissão}

A importancia de se analisar esses valores consiste em evitar sobrecarga das linhas, pois essas têm limilaçoes físicas que deverão ser respeitadas para que não haja interrupçôes na distributição da carga. Enn alguns trabalhos nessa área, as perdas nas linhas de transmissäo são comsideradas como funç̃o objetivo. Esses resultados são apresentados

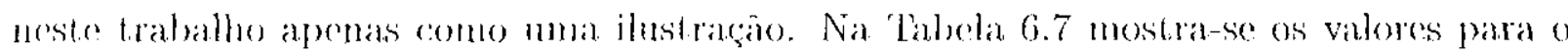
fluxo o para as pordas de potoncia notiva mas linbas.

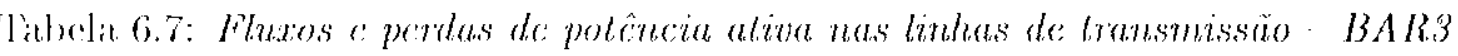

\begin{tabular}{|c|c|c|c|c|}
\hline \multicolumn{2}{|c|}{ linha } & \multirow{2}{*}{$\begin{array}{l}\text { fluxo } \\
(\mathrm{k}-\mathrm{m})\end{array}$} & \multirow{2}{*}{$\begin{array}{l}\text { fluxo } \\
(m-k)\end{array}$} & \multirow[t]{2}{*}{ perdas } \\
\hline (índice) & $(\mathrm{k}-\mathrm{m})$ & & & \\
\hline 1 & $1-2$ & 0,1854 & $-0,1844$ & $0,1034 \times 100-2$ \\
\hline 2 & $1-3$ & 0,4596 & $-0,4532$ & $0,6337 \times 10 \mathrm{c}-2$ \\
\hline 3 & $3-2$ & $-0,1807$ & 0,1824 & $0,1667 \times 10 \mathrm{e}-2$ \\
\hline
\end{tabular}

Os fluxos são methores visualizados através do gráfico da Figura 6.4

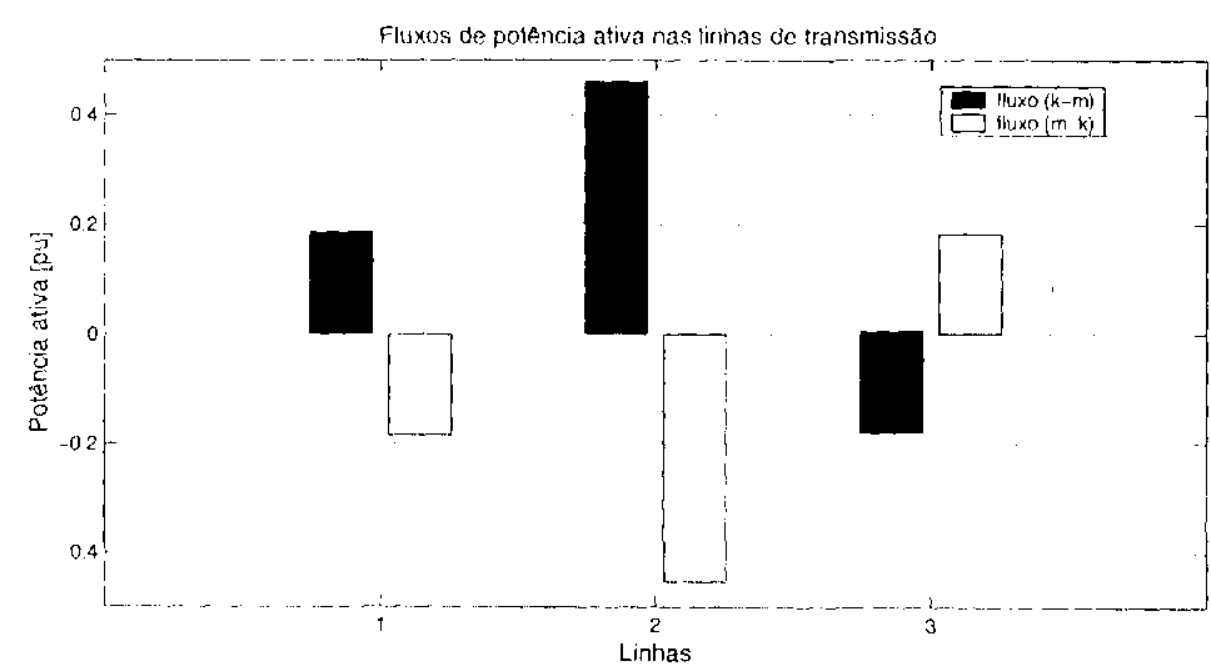

Figura 6.4: Flucos de potência ativa nas linhas de transmissäo-BAR3 (L-D) 


\subsection{IEEE30}

O IESE30 é un sistema ntilizado e aceito pelos pesquisadores na área como um problema para realização de testes em implementaçoes dos métorlos desenvolvidos. Devido principalmente à nĩo exploraçăo de sua estrutura esparsa de forma adecuaula pelit implementação feita em MATLAB, a utilização de pontos e parâmetros iniciais bascindos:

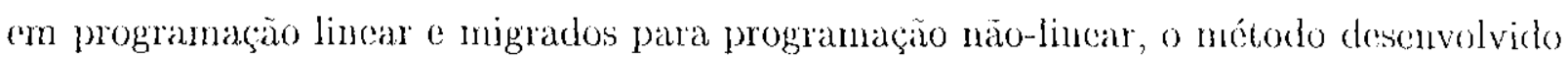
neste traballo tevo dificuldides para convergencia. A soluçăo foi atribuir o valor de a para $10^{-1}$ e realizar ajustes nos parametros para que pudessem compensar essas desvantigens. Outra mudança foi no linite míxinno de geração de potencia ativa para os exquemas l_-C

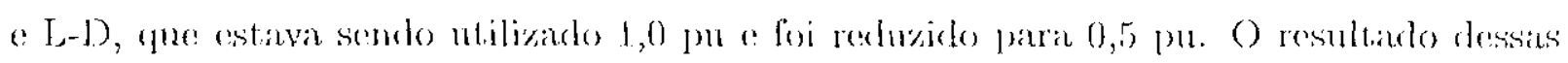
açôes foram satisfatórios e conseguiu-se realizar os mesmos testes que foram feitos para o sistema BAR3.

\subsubsection{Análises}

Pode-se observal que o programa comvergiu para todos os esquemas, nota-se também que o tempo computacional para a resolıção desse sistema foi muito pegueno. Estão também dispostos na Tabela 6.8 o múmero de iteraçōes necessárias e o número de operagoes realizadas para a convergencia do método.

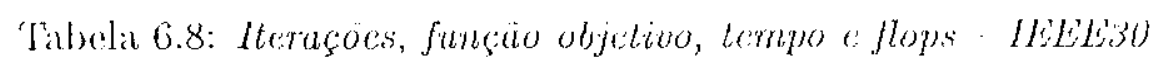

\begin{tabular}{c|c|c|c|c}
\hline esquema & iterações & $\phi$ & tempo [s] & flops \\
\hline L-A & 1 & 0,901 & 0,047 & 98900 \\
L-B & 1 & 0,899 & 0,047 & 98594 \\
L-C & 6 & 0,450 & 0,078 & 146187 \\
L-D & 10 & 0,718 & 0,125 & 245115 \\
L-E & 4 & 0,898 & 0,047 & 98435 \\
\hline
\end{tabular}

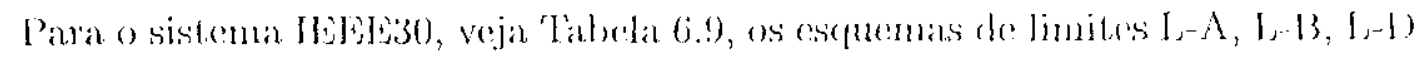

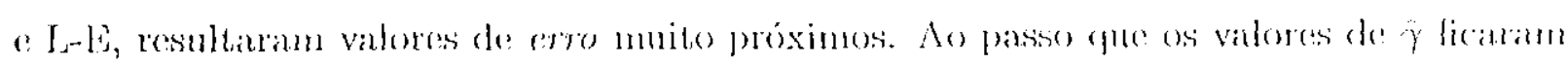




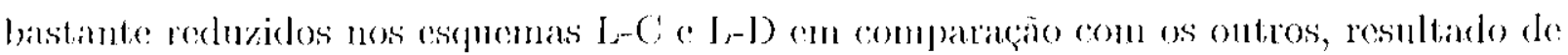
Ima maior restrição nos limites de tensão e geraçào de potência ativa nas barras.

Tabcla 6.9: Valor do erro e gap de complementaridade relativo - IWEE30

\begin{tabular}{|c|c|c|}
\hline esquema & erro & $\hat{\gamma}$ \\
\hline $\mathrm{L}-\mathrm{A}$ & $0,9697 \times 10 \mathrm{c}-1$ & $0.8532 \times 100-1$ \\
\hline $\mathrm{I},-\mathrm{B}$ & $0.9774 \times 100-1$ & $0,8331 \times 10 \mathrm{c}-1$ \\
\hline $\mathrm{I}, \mathrm{C}$ & $0,8.341 \times 100-1$ & $0,1957 \times 100-1$ \\
\hline $\mathrm{I},-\mathrm{D}$ & $0,9104 \times 10 \mathrm{c}-1$ & $0,2169 \times 100-3$ \\
\hline$I,-1 \%$ & $0,960 \pi \times 100-1$ & $0.7986 \times 100-1$ \\
\hline
\end{tabular}

\subsubsection{Tensão}

A tensão nas barras obtida no final das iteracoes para o sistema IEleE30, 11tilizando os esquemas acima citados, podem ser observadas na Tabela 6.10, onde a barra 16 a barra de referencia do sistema.

No gráfico da Figura 6.5 pode-se tor uma visualização da covolnção da tonsão

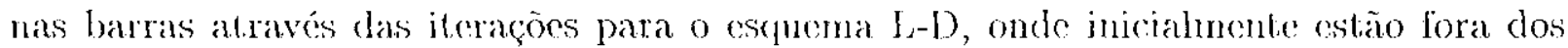
limites e são forçadas a 1,0 pu pola restrição imposta pelo esquema.

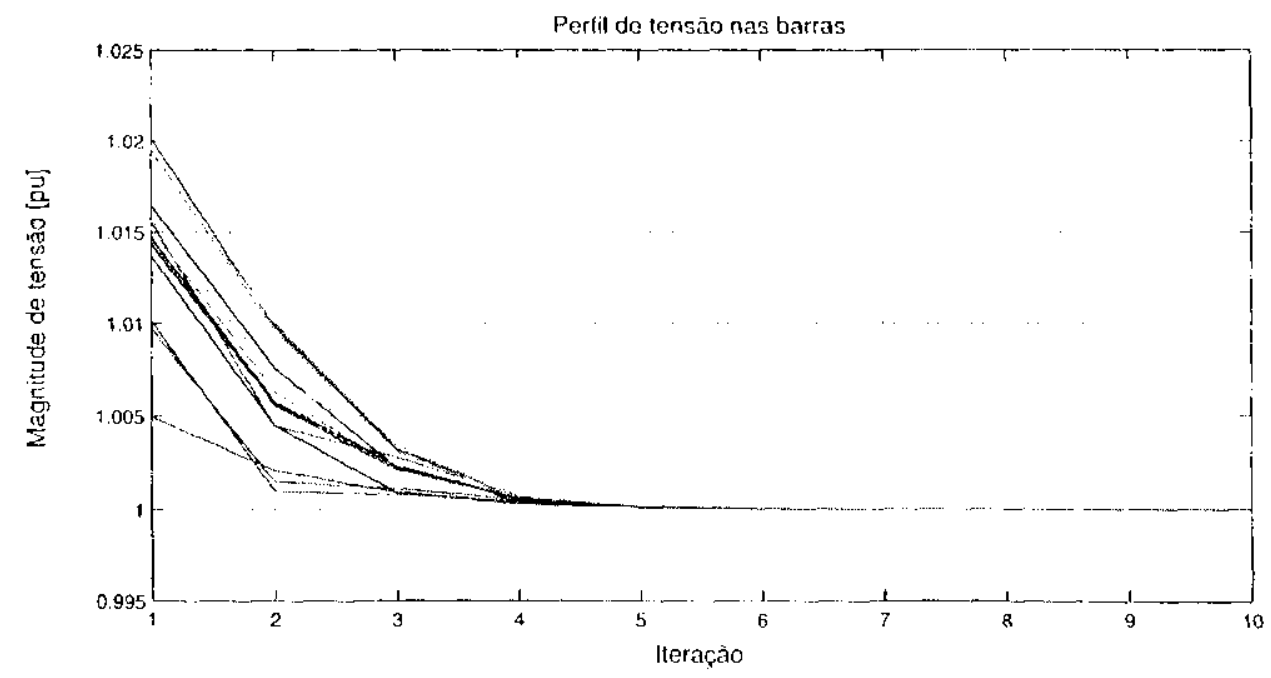

Figura 6.5: Tensäo nas barras - IEEE,30 (L-D) 
Tibola 6.10: Tensio nas bartas IEEL3O

\begin{tabular}{|c|c|c|c|c|c|}
\hline barra & L-A & $\mathbf{L}-\mathbf{B}$ & $\mathbf{L}-\mathbf{C}$ & L-D & L-F \\
\hline $1^{*}$ & 1,005 & 1,0000 & 0,996 & $1,(000)$ & 0,999 \\
\hline 2 & 1,011 & 1,0006 & 1,0009 & 1,000 & $1,00-1$ \\
\hline 3 & 1,006 & 1.0002 & 1,0000 & 1,6000 & 1,0001 \\
\hline 4 & 1,0007 & $\{, 003$ & 0,999 & 1,000 & 1,001 \\
\hline 5 & 0,998 & 0,995 & 1,0000 & 1,0000 & $0,99)^{3}$ \\
\hline (;) & 1,0006 & 1,002 & 0,998 & $1,(000)$ & $1,(001$ \\
\hline 7 & 1,0008 & 1,01066 & $1,(1000)$ & 1,0000 & 1,0605 \\
\hline 8 & 1,0606 & 1,0003 & $1,46 \%$ & $1,(6100)$ & $1,(00)$ \\
\hline 9 & 1,0001 & 0,997 & 0,991 & 1,0000 & 0,996 \\
\hline 10) & 1,007 & 1,0003 & 1,0601 & 1,0000 & 1,001 \\
\hline 11 & $1,0 ! 2$ & 1,0068 & 1,010 & 1.0000 & 1,6005 \\
\hline 12 & 1,0102 & 0,998 & 0,391 & 1,0000 & 0,996 \\
\hline 13 & 1,012 & 1,008 & 1,010 & 1,6000 & 1,000 \\
\hline 14 & 1,005 & 1,002 & 1,000 & 1,000 & $1,000)$ \\
\hline 15 & 1,0013 & 1,001 & 1,000 & 1,000 & 1,000 \\
\hline
\end{tabular}

\begin{tabular}{|c|c|c|c|c|c|}
\hline barra & L-A & L-B & $\mathrm{L}-\mathrm{C}$ & L-1) & $\mathbf{L}-\mathbf{E}$ \\
\hline 16 & 1,0005 & 1,002 & $1,(0) 00$ & 1,000 & $1,600)$ \\
\hline 17 & 1,0005 & 1,002 & $1,000)$ & 1,000 & 1,000 \\
\hline 18 & 1,005 & 1,0002 & 1,000 & 1,000 & 1,000 \\
\hline 19 & 1,005 & 1,0002 & 1,000 & $1,000)$ & $1,(1000)$ \\
\hline 211 & 1,005 & 1,0002 & 1,0001 & 1,600) & 1,0000 \\
\hline 21 & $1,(0105)$ & 1,0022 & $1,000)$ & $1,000)$ & $1,(m)$ \\
\hline 22 & 1,005 & 1,002 & 1,000 & 1,0100 & 1,0000 \\
\hline 23 & 1,0015 & 1,6022 & $1,(1)(x)$ & $1,(100)$ & $1,(0)(k)$ \\
\hline 24 & 1,005 & 1,002 & 1,0000 & $1,(000)$ & 1,000 \\
\hline 25 & 1,005 & 1,002 & 1,000 & $1,000)$ & 1,000 \\
\hline 26 & 1,005 & 1.002 & 1,000 & 1,000 & 1,000 \\
\hline 27 & 1,0066 & 1,002 & 1,001 & 1,0000 & 1,001 \\
\hline 28 & $1,0(0) 6$ & $1,000^{2} ?$ & 1,000 & $1,000)$ & $1,0(0) !$ \\
\hline 29 & $l, 005$ & 1,002 & 1,000 & 1,000 & 1,000 \\
\hline 30 & 1,005 & 1,002 & 1,000 & $1,(800)$ & 1,090 \\
\hline
\end{tabular}

\subsubsection{Potência Ativa}

Os resultados finais da injegão de potoncia ativa nes baras de geraça podem ser vistos na Tabela 6.11 .

A gevarano de potencia nas barras para o escuema I. D estão mostradas no grálico da Figura 6.6, onde as barras inicialmenter possuem valores próximos à fronteira da regiáo de lactibilidade. Ao longo das itcrações esses valores são ajustados em conjunto com as outras variáveis evitando cue essas ultrapassem os limites dessia região. 


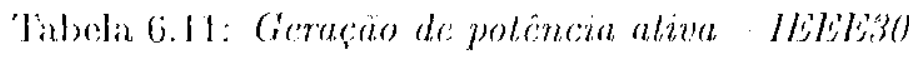

\begin{tabular}{c|c|c|c|c|c}
\hline barra & T-A & L-B & L-C & L-D & L-E \\
\hline 1 & 0,549 & 0,548 & 0,384 & 0,480 & 0,544 \\
2 & 0,563 & 0,562 & 0,391 & 0,478 & 0,559 \\
5 & 0,555 & 0,555 & 0,394 & 0,488 & 0,553 \\
8 & 0,536 & 0,537 & 0,383 & 0,496 & 0,540 \\
11 & 0,543 & 0,543 & 0,386 & 0,497 & 0,541 \\
13 & 0,542 & 0,542 & 0,386 & 0,495 & 0,542 \\
\hline
\end{tabular}

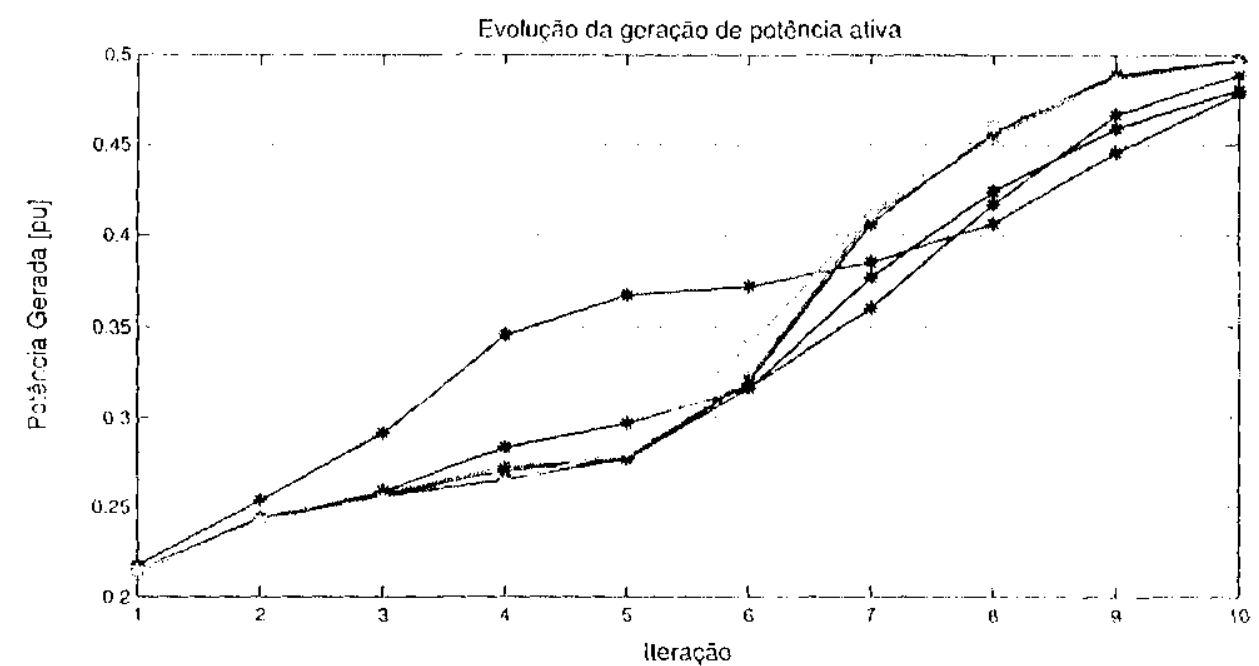

Figura 6.6: Geraçäo de potoncia aliva - LELE30 $(L-D)$

\subsubsection{Normas Residuais Relativas}

As nomas residuais iniciais possucm valores altos, causado pelo ponto inicial de operagra obtido do resintado do programa de fluxo de carga, que somente está reblacionado com as igualdades do problema, por isso as normas residuais referentes a $r p$ e $r q$ são praticamente zero na inicialização e permanecen inalteradas ao longo das iteraçós, ao contrário de $r v$ e rs que representam as restriçoes canalizadas incorporadas no problema. No gráfico da Figura 6.7 pode-se notar que esses valores decresem para zero, atemdendo dessia forma todas as restriçöes do problema. 


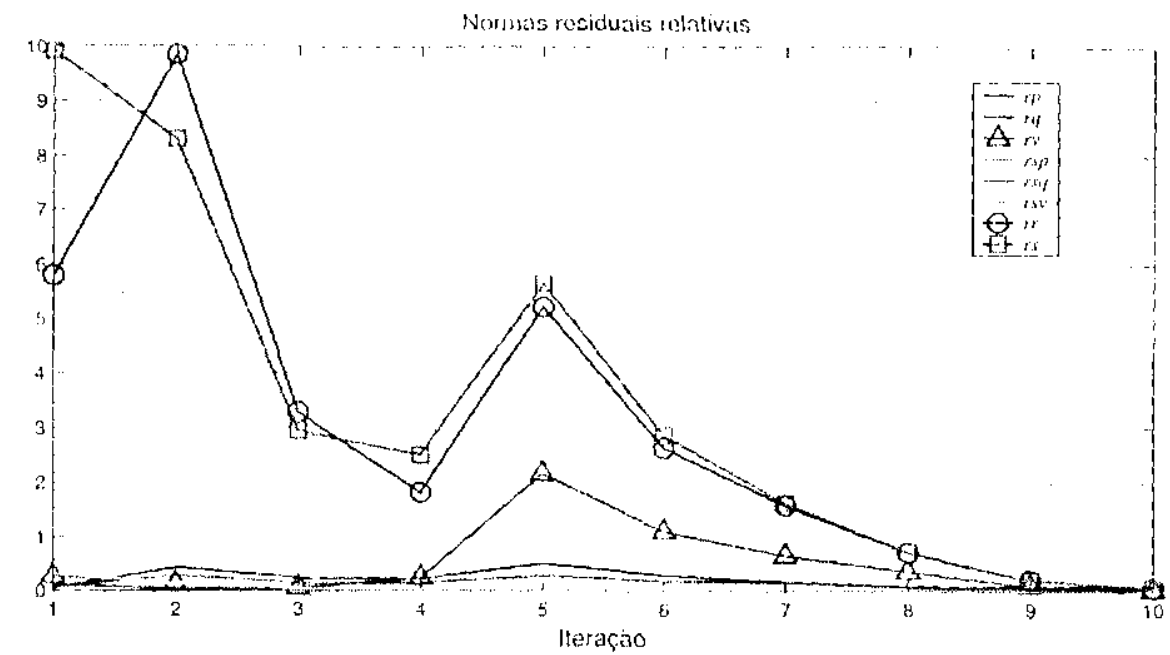

Figura 6.7: Normas residuais relativas IEEEso $\left(L_{-}-D\right)$

\subsubsection{Fluxo e Perda de Potência Ativa nas Linhas de}

\section{Transmissão}

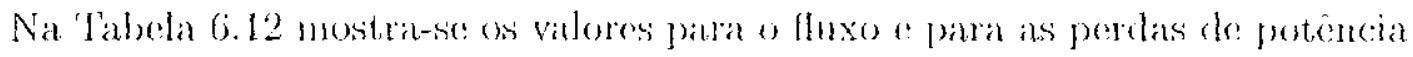

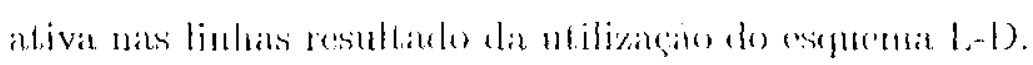

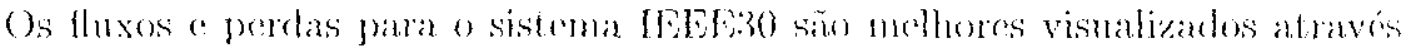

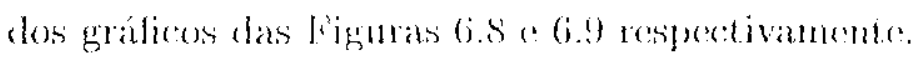

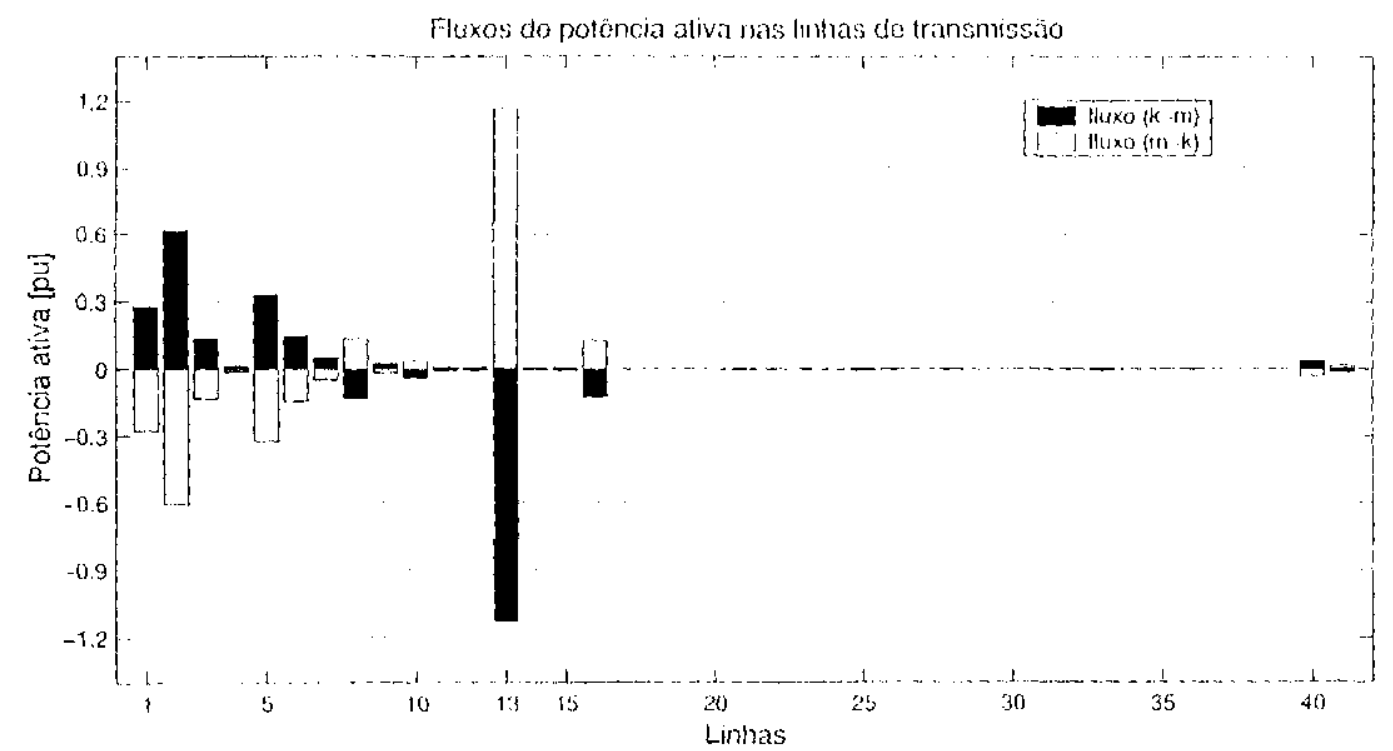

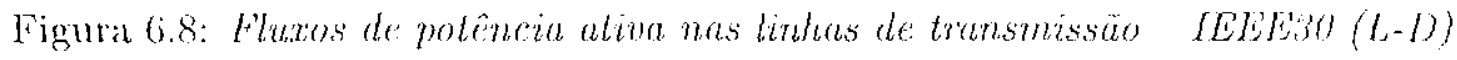




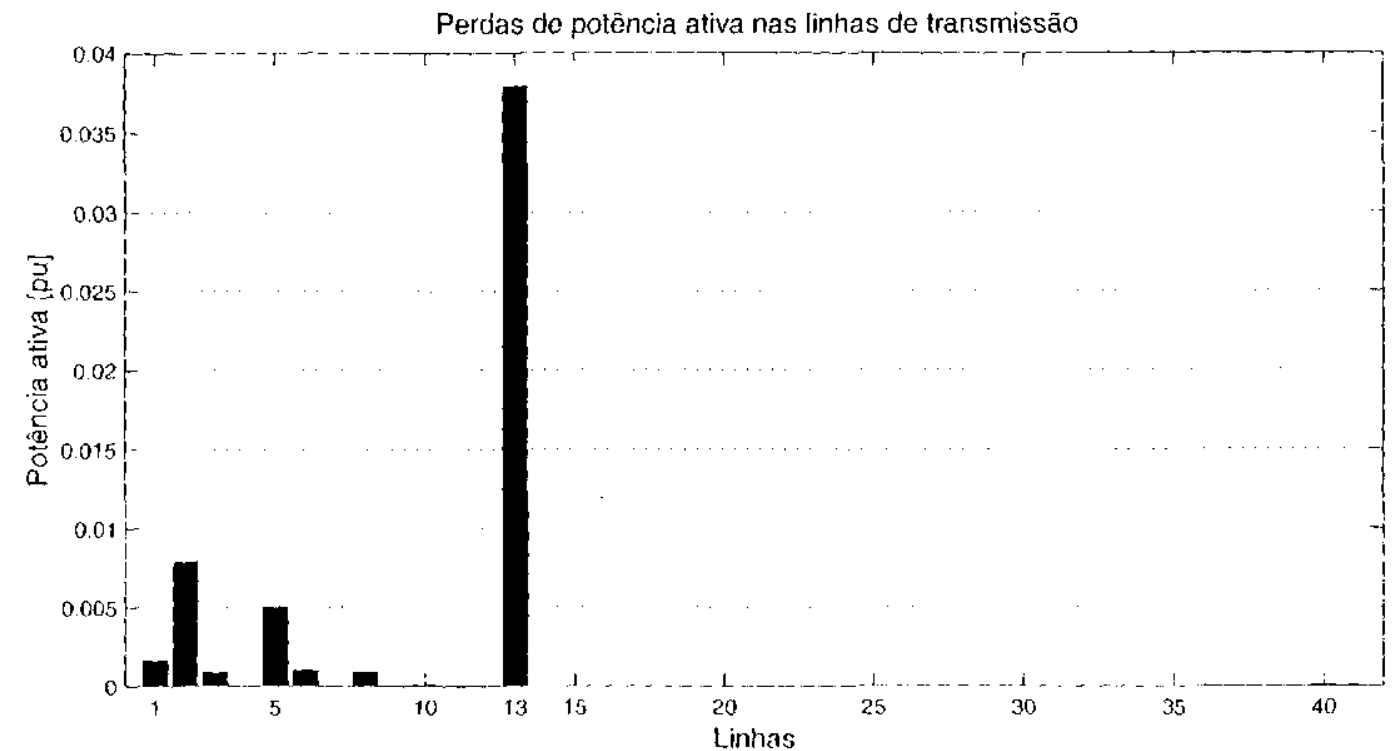

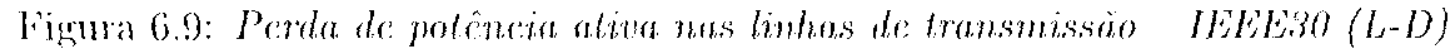

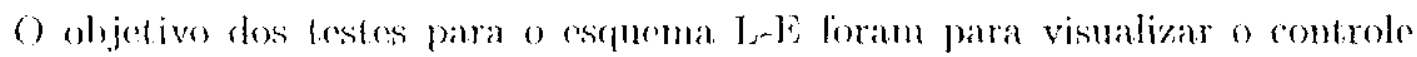

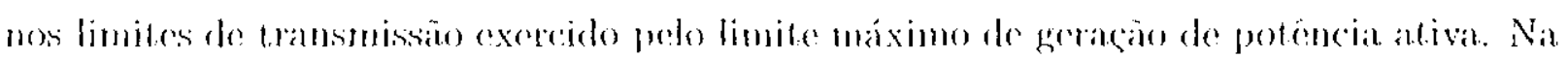
Jabola 6.l3 cxibe-se os fluxos nas limhas para cala limite máximo de geração de potencia atival.

Pode-se notar que devido à esse controle no limite máximo de potencia a ser grata, os vatores dos fluxos quando tendem a diminuir conforme esse limite. O gráficos das Figuras 6.10 e 6.11 mostram o comportanento dos fluxos de potencia ativa nas linhas

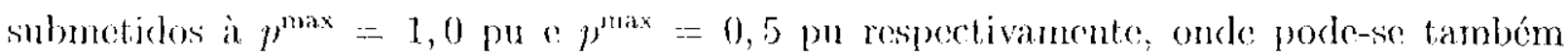
notar que para o primeiro limite, o valor do ftuxo do potencia ativa na linha 13, entre as barass 11 o 9 6 de 1,295 pu. P’an o segundo limite houve uma redução significativa desse valor para 0,8249 pu. Conseqüentemente as perdas também são reduzidas, veja nos mráficos das fïguras 6.12 a 6.13 


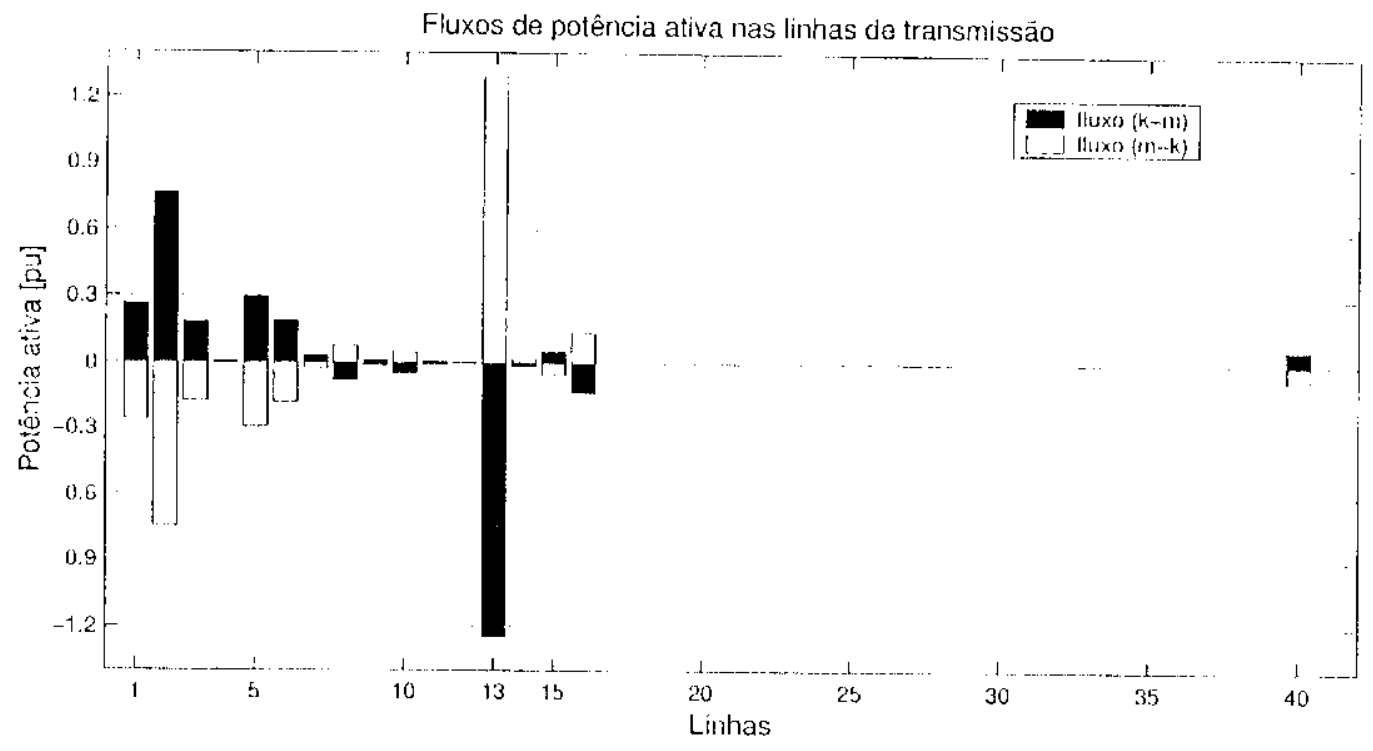

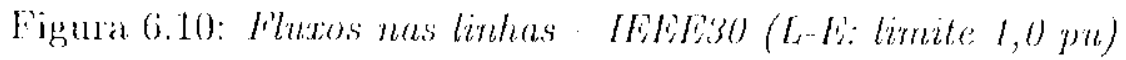

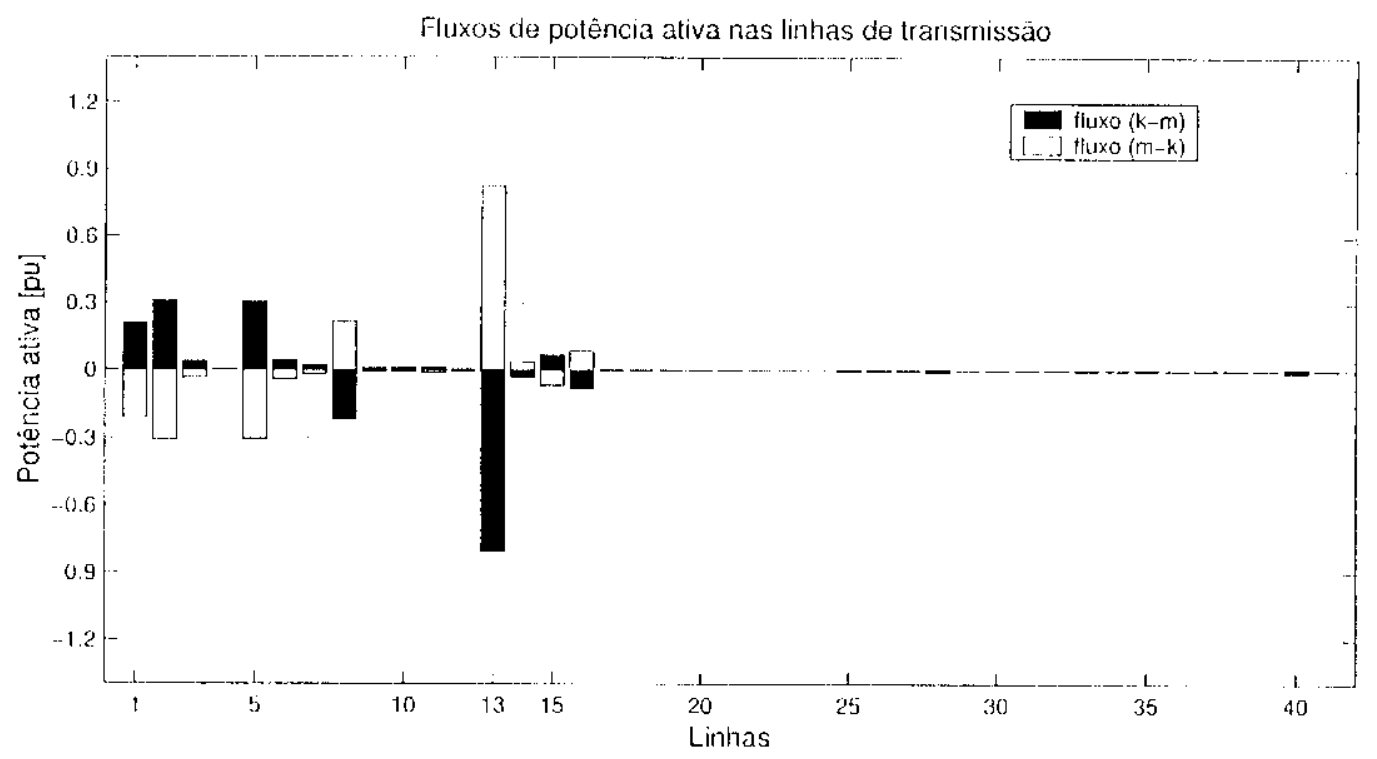

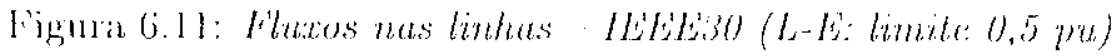




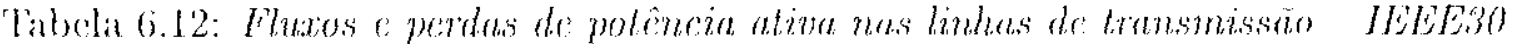

\begin{tabular}{|c|c|c|c|c|}
\hline \multicolumn{2}{|c|}{ linhn } & \multirow{2}{*}{$\begin{array}{l}\text { fluxo } \\
(k-311)\end{array}$} & \multirow{2}{*}{$\begin{array}{l}\text { fluxo } \\
(111-k)\end{array}$} & \multirow[t]{2}{*}{ perrlas } \\
\hline (incliece) & $(\mathrm{k} \cdot \mathrm{mi})$ & & & \\
\hline 1 & $1-2$ & 0,2773 & $-(0,2757$ & $0,1580 \times 10 \mathrm{e}-2$ \\
\hline 2 & $1-3$ & 0,6163 & -0.6084 & $0,7894 \times 10 \mathrm{k}-2$ \\
\hline 3 & $2-4$ & 0,1316 & $-0,1338$ & $0,8671 \times 10 \mathrm{c}-3$ \\
\hline 4 & $3-4$ & 0,01214 & $-0,01213$ & $0,8653 \times 100-5$ \\
\hline i) & $2-5$ & 0,3287 & -0.3237 & $0,4098 \times 10 \mathrm{c}-2$ \\
\hline 6 & $2-6$ & 0,1465 & $-(0,1455$ & $0,1014 \times 10 \mathrm{e}-2$ \\
\hline$i$ & 4.6 & $0,65,5061$ & $-10,0,50557$ & $0,331.3 \times 100 \ldots 1$ \\
\hline 8 & 5.7 & $-11,1338$ & 0,1346 & $0,8755 \times 100-3$ \\
\hline 9 & $6-7$ & 0,02033 & - & $0,23332 \times 100-4$ \\
\hline 10 & $6-8$ & $-0,0375$ & 0,03759 & $(0,8915 \times 10)(-4$ \\
\hline 11 & $6-9$ & -0.004069 & $0,010.1971$ & $0,1411 \times 100-5$ \\
\hline 12 & $6-10$ & $-0,006363$ & 0,006366 & $0,2343 \times 100-5$ \\
\hline 13 & $9-11$ & $-1,128$ & 1,166 & $0,3795 \times 10)(-1$ \\
\hline 14 & $9-10$ & $-10,002997$ & 0,0012998 & $0,2588 \times 10 \mathrm{r}-6$ \\
\hline 15 & $4-12$ & 0,006619 & $-0,006618$ & $0,1312 \times 10 r-5$ \\
\hline 16 & $12-13$ & $-0,1264$ & $(1,1264$ & $(0,0)$ \\
\hline 17 & $12-14$ & 0,0005006 & $-0,0001006$ & 0,0 \\
\hline 18 & $12-15$ & 0,0001957 & $-0,0001957$ & 0,0 \\
\hline 19 & $12-16$ & $0,000428.1$ & 0,60004284 & 0,0 \\
\hline 20 & $14-15$ & 0,0004809 & -0.00048090 & 0,0 \\
\hline 21 & $16-17$ & 0,00166 & $-0,00160$ & 0,0 \\
\hline 22 & $15-18$ & 0,0006443 & $-19,00006413$ & 0,0 \\
\hline 23 & $18-19$ & 0,010066 & $-0,000166999$ & $0,2946 \times 160-7$ \\
\hline 24 & $19-20$ & 0,0002488 & $-0,0002188$ & $0,5675 \times 10 \mathrm{c}-8$ \\
\hline 25 & 11). 21$)$ & $-(0,010), 3928$ & $0,0(133929$ & $0,1977 \times 196-10$ \\
\hline 26 & $10-17$ & $-0,002434$ & 0,002434 & $0,6968 \times 10 \mathrm{kc}-7$ \\
\hline $2 i$ & 10-21 & $-0,001511$ & $0,(6) 1511$ & $0,2730 \times 100.7$ \\
\hline 28 & $10-22$ & $-0,001972$ & 0,001972 & $0,4599 \times 10 e^{-7}$ \\
\hline 29 & $2 \mathrm{~J}-22$ & $-0,000101$ & 0,0000101 & $0,5191 \times 10 \mathrm{c}-9$ \\
\hline 30 & $15-23$ & 0,00022 & $-0,00022$ & 0,0 \\
\hline 31 & $22-24$ & $-0,(101035$ & 0,0101036 & $0,1179 \times 10 \mathrm{e}-6$ \\
\hline 32 & $23-24$ & 0,0006103 & $-0,0006103$ & $0,23 \pi 4 \times 10 \mathrm{e}-7$ \\
\hline 33 & $24-25$ & $-0,130 \cdot 1222$ & 0,001223 & $0,7945 \times 10 k-6$ \\
\hline 34 & $25-26$ & $-0,8774 \times 100-4$ & $0,8774 \times 10 e-4$ & $0,6175 \times 10 \mathrm{c}-8$ \\
\hline 35 & $25-27$ & $-0,000811$ & 0,0008111 & $0,1091 \times 10_{e}-6$ \\
\hline 36 & $28-27$ & 0,0301998 & $-0,001997$ & $0,136 \mathrm{i} 0 \times 10 \mathrm{c}-5$ \\
\hline 37 & $27-29$ & $-10,0003001$ & 0,0003001 & $0,2478 \times 10 \mathrm{e}-7$ \\
\hline 38 & $27-30$ & $-0,0003371$ & 0,0003372 & $0,3129 \times 10 \mathrm{e}-7$ \\
\hline 39 & $29-30$ & $-0,00011232$ & 0,0001232 & $0.1250 \times 100-8$ \\
\hline 40 & $8-28$ & 0,103509 & $-0,03502$ & $0,6595 \times 10 \mathrm{c}-4$ \\
\hline 41 & $6-28$ & -0.0134 & 0,01341 & $0.1098 \times 10,-4$ \\
\hline
\end{tabular}




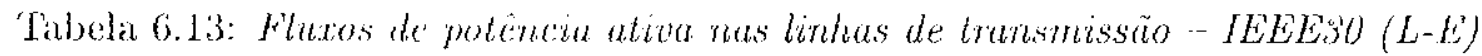

\begin{tabular}{|c|c|c|c|c|c|}
\hline \multicolumn{2}{|c|}{ linha } & \multicolumn{2}{|c|}{$\left.p^{\max }=1, g_{\mathrm{p}}\right) \mathrm{u}$} & \multicolumn{2}{|c|}{$p^{\max }=0,5 \mathrm{pu}$} \\
\hline (índice) & $(k-111)$ & fluxo $(k-m)$ & Hnxo $(n n-k)$ & fluxo $(k-\mathrm{m})$ & fluxo $\{m-k\}$ \\
\hline 1 & $1-2$ & 0,20053 & $-13,2633$ & $0,210.1$ & $-(1,2086$ \\
\hline 2 & $1-3$ & $0,76.4$ & $-0,7513$ & 0,3111 & $-0,3089$ \\
\hline 3 & $2-1$ & $0,1 \times 19$ & $-01,1803$ & 0,033618 & $-0,01360108$ \\
\hline l & $3-4$ & $0,00292 !$ & $-0,60292$ & 0,001214 & $-40,601213$ \\
\hline 5 & $2-5$ & 0,2976 & $-0,2937$ & 0,3086 & $-0,3043$ \\
\hline 6 & $2-6$ & 0,1888 & $-0,1871$ & $0,(0.4099$ & $-0,04087$ \\
\hline 7 & $4-6$ & 0,02981 & $-(0,0298$ & 0,0211 & $-(0,021409$ \\
\hline 8 & $5-7$ & $-10,07821$ & 0,07801 & $-0,21+2$ & 0,2161 \\
\hline 9 & $6-7$ & 0,01214 & $-0,01200$ & 0,007306 & $-0,007285$ \\
\hline 10 & $6-8$ & $-(0,0) \cdot 871$ & 0,04886 & $-0), 1008198$ & 0,008202 \\
\hline 11 & $6-9$ & $0,(1066581$ & $-0,0060546$ & 0,0127 & $-0,0126$ \\
\hline 12 & $6-10$ & $-1), 0018$ & 0,0018 & $-0,003307$ & 0,0013309 \\
\hline 13 & $9-11$ & $-1,249$ & 1,295 & $-0,8071$ & 0,8249 \\
\hline 14 & $9-10$ & $-0,01776$ & 0,01781 & $-0,03383$ & 0,03412 \\
\hline 15 & $4-12$ & $0,05,37$ & $-0,053348$ & 0,0666533 & $-0,06661$ \\
\hline 16 & $12-13$ & $-0,1371$ & 0,1371 & $-0,08364$ & 0,08364 \\
\hline 17 & $12 \cdots 1.1$ & $\times(03,5) \times 106-5$ & $-8,6335 \times 364-5)$ & $-7,935 \times 10)(-5)$ & $7,9355 \times 19)(-5)$ \\
\hline ו & $12-15$ & 0,00061576 & $-10,00001576$ & $-2,874 \times 100,5$ & $2,874 \times 16(2-5)$ \\
\hline 19 & $12-16$ & 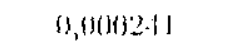 & $-10,00 m+211$ & $1,335 \times 14 k: 5$ & $-1,3355 \times 103 \ldots-5$ \\
\hline 20 & $14-15$ & 0,40903924 & $-4,(60139424$ & 0,00012575 & $-0,00602575$ \\
\hline 21 & $16-17$ & $(1,(000) 93,372$ & $-41,(1400093372$ & $0,(07004.155$ & $-0,0000-4155$ \\
\hline 22 & $15-18$ & $0,00013.3 .160$ & $-(3,0) 003,160$ & $(1,(0) 00) \cdot 14 i)$ & $-11,00311.403$ \\
\hline 23 & $18-19$ & 0,00003171 & $-(0,(6) 0033171$ & 0,0000172 & $-0,000172$ \\
\hline $2: 1$ & $19-20$ & $9,4312 \times 136-i)$ & $-9,612 \times 100-5)$ & $3,217 \times 104=5$ & $-3,217 \times 30 k-5$ \\
\hline 25 & $10-20$ & 0,013112 & $-0,(6) 1118$ & $0,\{30319.16$ & $-0,0003943$ \\
\hline 26 & $30-17$ & $0,001 \cdot 40.4$ & $-0,0001103$ & 0,004055 & $-(0,004052$ \\
\hline 27 & $10-21$ & 0,00256 & $-0,002559$ & 0,0096657 & $-0,0014654$ \\
\hline 28 & $10-22$ & 0,002552 & $-0,(10) 2551$ & $0,061.1726$ & $-0,004723$ \\
\hline 29 & $21-22$ & $-2,199 \times 16 \mathrm{e}-6$ & $2,199 \times 10 \mathrm{e}-6$ & $1,431 \times 100-5$ & $-1,431 \times 10 e-5$ \\
\hline 30 & $15-23$ & 0,0003185 & $-03,0003 \cdot 185$ & $0,(3001301$ & $-0,0001394$ \\
\hline 31 & $22-24$ & $-0,00002451$ & 0,0002451 & $-0,0004046$ & 0,0004047 \\
\hline 32 & $23-24$ & 0,00004571 & $-0,0004571$ & $-0,0002384$ & 0,0002386 \\
\hline 33 & $24-25$ & 0,001662 & $-0,001662$ & $0,00187\}$ & $-0,001871$ \\
\hline $3-1$ & $25-26$ & $3,872 \times 10 \mathrm{k}:-5$ & $-3,872 \times 100-5$ & $3,284 \times 100-5$ & $-3,284 \times 100.5$ \\
\hline 35 & $25-27$ & $-13,0001072$ & 0,0001071 & $-8,,(0005873$ & 0,0005875 \\
\hline 36 & $28-27$ & $-0,0000: 3113$ & 0,00034143 & $-0,6903777$ & 0,0003777 \\
\hline 37 & $27-29$ & 0,0009882 & $-0,0009881$ & 0,002101 & $-0,0021$ \\
\hline 38 & $27-30$ & 0,00101933 & $-0,(60) 1003$ & $0,(w) 2113$ & $-0,002112$ \\
\hline 39 & $29 \cdot 30$ & $1,54 \times 30 \mathrm{e}-5$ & $-4,34 \times 16 e-5$ & $3,735 \times 10 \mathrm{e}-5$ & $-3,735 \times 100-5$ \\
\hline 40) & $8-28$ & 0,07074 & $-0,07048$ & 0,0090763 & $-0,009059$ \\
\hline 41 & $6-2 x$ & $-(1,000033932$ & 0,0063933 & $-0,0002026$ & 0,0021327 \\
\hline
\end{tabular}




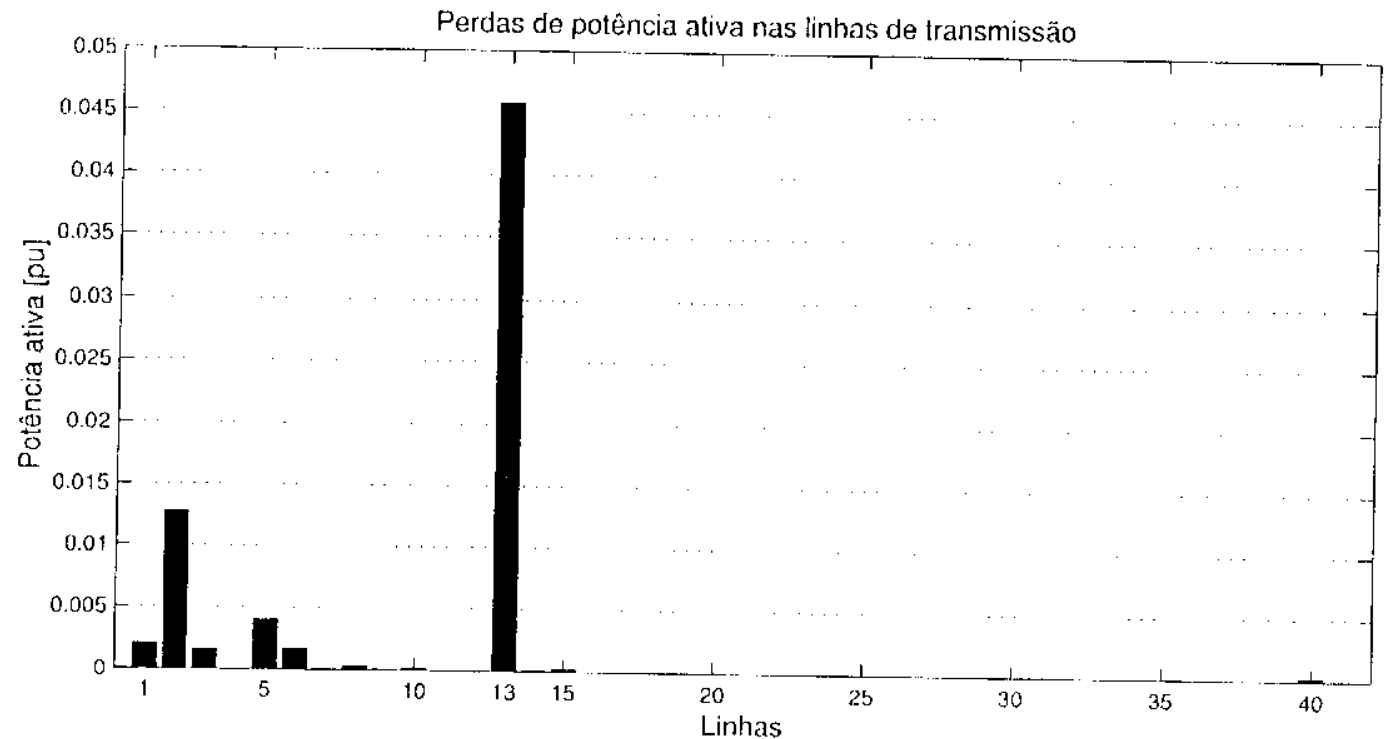

Figura 6.12: Perda nas linhas de transmissäo - IEEE30 (L-E: limite 1,O pu)

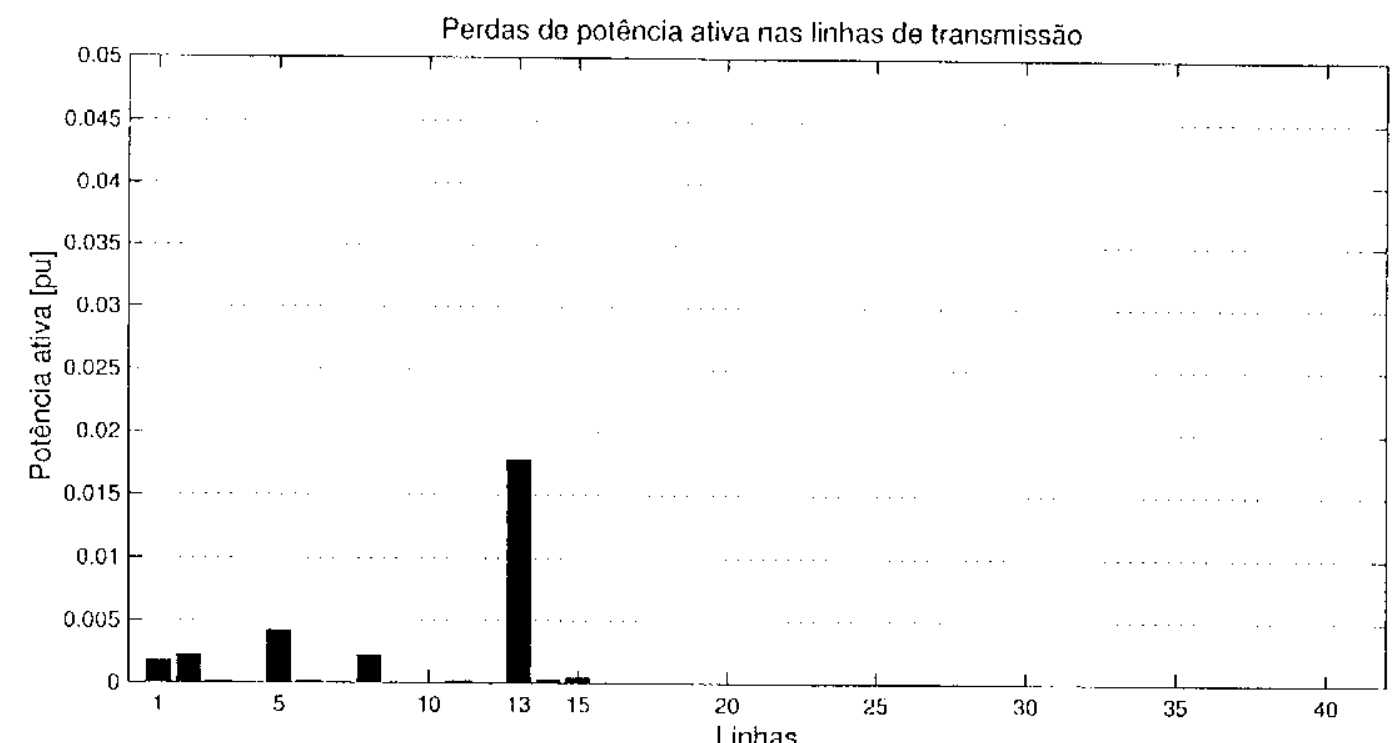

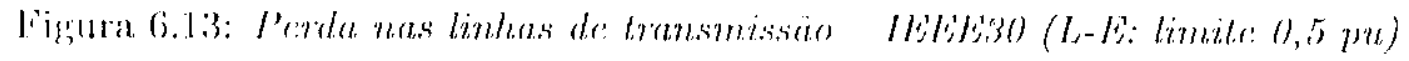




\section{Capítulo 7}

\section{Conclusões}

Os resultados indicam que os métodos de pontos interiores säo promissoress jatra estal classe de problemas.

Pode-se observar que as iteraçōes do método são rápidas. Esta velocidlade é

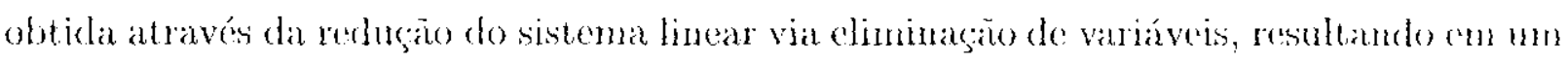
sistema cuja dimensão comesponde a duas vezes o número de barras do problema original.

É importante salientar que a utilização de coordenadas cartesianas contribuiu parra o desenvolvimento do método fornecendo Jacobianas menos complexas e contribuindo

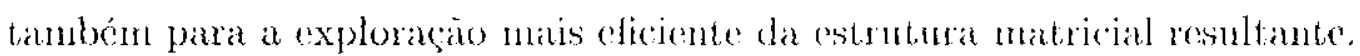

A estrutura dia mortelagem permitiu a imposição de fortes restriçôes ì algumas variáveis sem o aumento do tempo e operaçoes de ponto flut nante, contribuindo para um

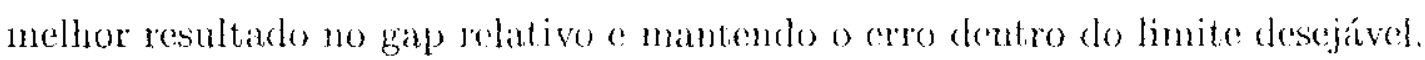

Conseguiu-se obter bons resultados nas perdas de potencia ativa nas linhas do transmissio, mesmo nizo sendo o objetivo do modelo ntilizado. Esse resultado mostia de

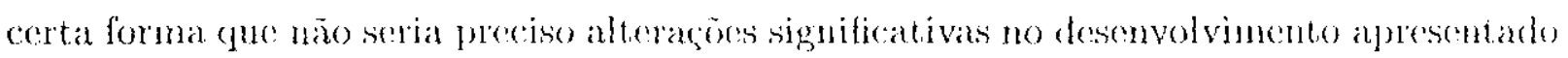
neste trabatho para se adotar outras fincooes objetivo.

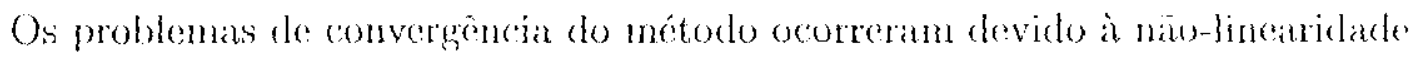
do problema de fluxo de carga ótimo $\mathrm{AC}$, dificultando a escoltha de paramouros e pontos iniciais adequados. A utilizaço de pontos initiais resultantes do problema de fluxo de carga estäo relacionadas apenas com as erguagoes de fhuxo de carga, sendo necossário ajustes referentes às ontras restriçōes do problena. 
Estes métorios porlem ser implementados para resolver problemas de grande

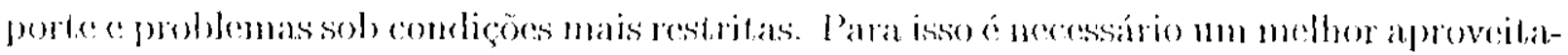
mento din cestrutura esparsa dos problemas e também un estudo mais detalhado sobre a

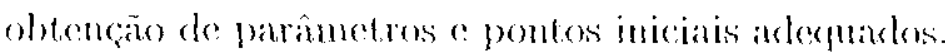




\section{Capítulo 8}

\section{Propostas Futuras}

Unia proposta para a contimudade desse projeto o a traducano desta implementação para uma linguagem de computaçio mais poderosa, onde pudessem ser exploradas, a estrutura esparsa do problema e as caracteristicas da matriz do sistema linear resultante tais como, ser estruturahnente simćtrica e ter uma estrutura originada de una rede de transmissão, considerando transformadomes defasadores. Juntamente con essa implementação, a criação do uma estrutura de dades eliciente, reduzindo dessia formano número de operaços realizadas o o tempo computaciomal.

Ontra moditicaçäo seria adicionan outras característicals ao modelo, tais conm watanento de limites de transmissĩo de potencia ativa e diferentes funçós objetivo, de forma in obter uma implementação robusta, eficiente e que possa representar sistemas de potencia reais.

Fxiste a possibilidade de algumats mudanças no desonvolvinento do mótodo

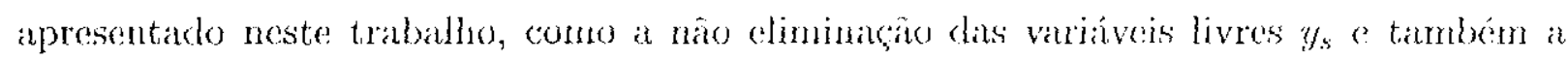
näo criação das variáveis de folga $z_{s}$. Fn alguns trabalhos ne'ssa área, pode-se cucontrat

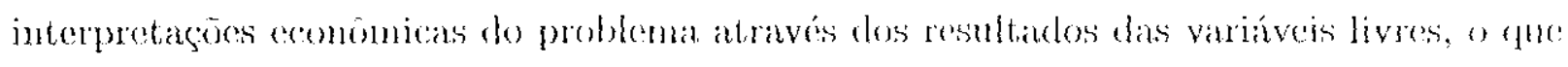
lanbém pode ser estuclato posteriorneme. 


\section{Bibliografia}

[1] I. Ableb, M. (i. C. Rishinde, G. Veilia, and N. Kakmarkar, Am implementhtion of Karmarkar's algorthm for linear programming, Mathematical Programming, 44 (1989), pp. 297335.

[2] J. E. Dennis and R. B. Scunaliel, Numerical Methods for Unconstrained Optimization and Nonlinear Fquations, SIAM, Philadelphia, PA, 1996.

[3] A. S. El-Bakmy, R. A. TAmin, T. Tsuchiya, and Y. Zhang, On the formalntion and the theory of the Newton interior-point method for nonlinear programming, Journal of Optimization l'hery and Applications, 89 (1996), p). 507.541

[4] A. V. Fiacco AND G. P'. MCConmick, Nontinear Programming: Sequential Unconstruined Minimization Techniques, John Wiley, New York, 1968.

[5] K. R. FRrscn, The logarithmic potential method of convex programming, menorandum, University Justitute of Economies, Oslo, Norway, 1955.

[6] G. H. Golun AN1) C. F. VAN LOAN, Matrix Computations Thitd Édition, The Johns Llopkins University Press, Baltimore, Maryland, 1996.

[7] J. Gondzio, Presolve analysis of linear proyramming prior to applying an interion point method, ORSA Jumnal on Computing, 9 (1997), pp). 73-91.

[8] S. Granvibue, Optimul reactive pouter dispateh through interior point methods, IEE⿰亻 Transactions on Power Systems, 9 (1994), p). $136 \cdot 146$.

[9] D. G. Luenberaer, Linear and Nonlinear Programming, Addison-Wostey, Reading, 1984. 
[10] I. .J. Lustig, R. F. Marstern, and D. F. Shanno, Computational experience with a primal-dual interior-point method for linear programming, Lincar $\Lambda$ lgebra Ap1l., 152(1991), p). 191-222.

[11] _-, On implcmenting Mehrotra's predictor-corrector interior point method for lincar proyramming, SI M J. Optimization, 2 (1992), pp. $435-449$.

[12] J. M. MARTINEZ AND L. 'T. SANTOS, Some new theoretical results on recursive quadratic programming algorilhms, Journal of Optimization Theory and Applications, 97 (1998), pp. 435-454.

[13] S. MEURotra, On the implementation of a primal-dual interior point method, SIAM Journal on Optimization, 2 (1992), pp). $575 \cdot 601$.

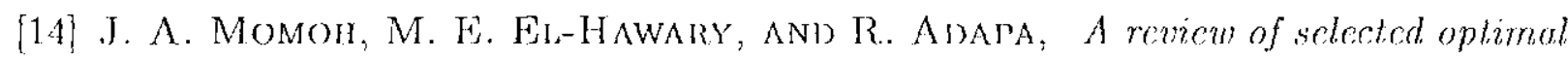
power flow literature to 1993 , part 11 Newton, linear programming and interior point methods, IEEE Transactions on l'ower Systems, 14 (1999), pp. 105111

[15] R. D. C. Monteiro, I. Abler, and M. G. C. Resende, A polynomial-time primal-dual affine scaling algorithm for linear and convex quadratic programming and its power series extension, Mathematics of Operations Research, 15 (1990), pp. 191 214.

[16] A. J. Mon'ticelli, Fluxo de Carga em Redes de Energia Elétrica, Edgard Blücher, São Paulo, 1983.

[17] A. R. L. Otiverra, L. Nepomuceno, and S. Soares, Short term lydroelectric scheduling combining network, flow and interior point approaches, Traballo submetido à Electrical Power \& Energy Systems, (2001).

[18] _..., Optimal active power dispatch combining network flow and interior point approaches, Accito para publicarã̃o, IEEE Transactions on Power Systems, (2003).

[19] A. R. L. Odiveira nND S. SonRes, Mélodos de pontos interiores para problema de fauto de polêncio ótimo, Anais do XIII Congresso Brasileiro de Antomática, em 
CD-ROM, Floriampolis, SC, aceito para publicagão na SBA: Controle \& Automag̃o nat condigaio de antige convidado, $(2000)$, pp). $790 \cdots 795$.

[20] V. H. Quindana, G. L. 'Tormes, and J. Medina-Patomo, Interior point methods and their applications to power systems: A classification of publications and software codes, IELE Thansactions on Power Systems, 15 (2000): IPp. 170 - 176.

[21] S. SoARes and C. T'. SAlmazo, Mintmum loss predispateh model for hydrotelelyc systems, IEEE Transactions on Power Systens, 12 (1997), pp. 1220 -1228.

[22] R. A. TAPIA AND Y. ZHANG, Superlinear and quadratic convergence of primaldual intertor point wethods for lineser programming revisited, Journal of optinization Theory and Applications, 73 (1992), pp. $229-242$.

[23] G. I. TORRES AND V. H. QUUNAANA, An interior point method for nonlinear optimal pouner flow using voltage relangular coordinates, IEEE Transactions on Power Systoms, 1:3 (1998), PJ). 1211 1218.

[24] R. J. VANDERBBE, Lincar Progremming Funndations and Halensions, Khuwer Acadennics P’ublishons, Boston, USA, 1996.

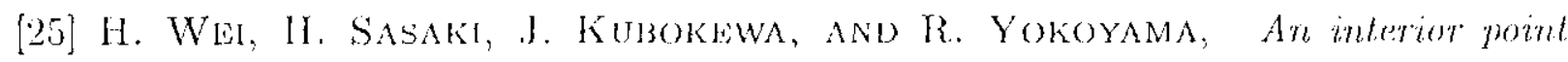
nonlinear programming for optimal power flow problems with a novel data struature, IELE Thanactions on Power Systems, 13 (1998), pp. 870.877.

[26] S. J. Wright, Primal Dual Futerior-Point Methods, SIAM Publications, SIAM, Philadelphia, PA, USA, 1996.

[27] Y. Q. WU, A. S. I)EJSS, AND R. E. MASTERN, A direct nontinetur predictorcorrector primal-dual interior point algorithm for optimal power flows, IVEE Thansactions on Power Systens, 9 (1994), pp. 876883. 


\section{Apêndice}

\section{Relatórios de Saída da Implementação}

Nesse anexo do trabalho sĩo mostrados os relatórios de saída que foram gerados pela execução do programa para os sistemas BAR3 e IEEE30, utilizando os esquemas para limites de tensão eonforme deserito anteriomente. Esses relatórios contém valores inicians de parámetros e os resultados provenicntes da última iteracão executada.

\section{BAR3 - Esquema L-A}

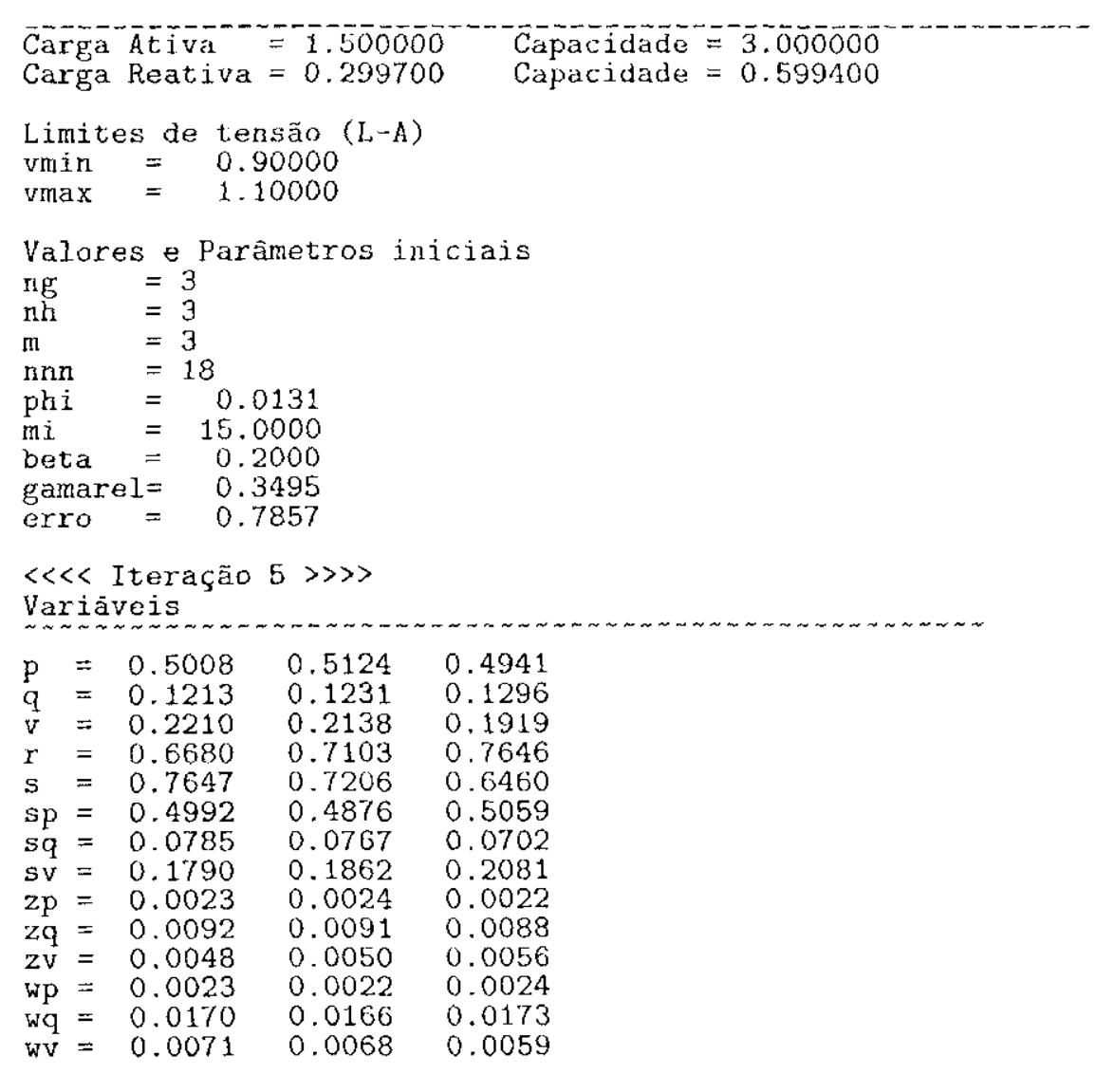


Direções

$\begin{array}{lrrr}\mathrm{dp}= & =0.0018 & -0.0103 & 0.0090 \\ \mathrm{dq}=0.0023 & 0.0016 & 0.0008 \\ \mathrm{dv}=0.0061 & 0.0060 & 0.0049 \\ \mathrm{dr}=-0.0002 & 0.0006 & 0.0017 \\ \mathrm{ds}=0.0034 & 0.0027 & 0.0004 \\ \mathrm{dsp}=-0.0018 & 0.0103 & -0.0090 \\ \mathrm{dsq}=-0.0023 & -0.0016 & -0.0008 \\ \mathrm{dsv}=-0.0061 & -0.0060 & -0.0049 \\ \mathrm{dzp}=-0.0063 & -0.0059 & -0.0068 \\ \mathrm{dzq}=-0.0176 & -0.0181 & -0.0193 \\ \mathrm{dzv}=-0.0153 & -0.0158 & -0.0171 \\ \mathrm{dwp}=-0.0065 & -0.0070 & -0.0060 \\ \mathrm{dwq}=-0.0762 & -0.0763 & -0.0794 \\ \mathrm{dwv}=-0.0186 & -0.0181 & -0.0159\end{array}$

Resíduos

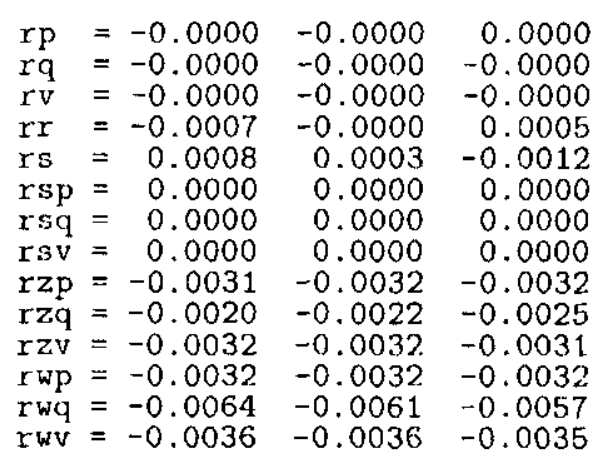

Normas

$\operatorname{norm}(\mathrm{rp}) / \mathrm{np}=0.0000$

$\operatorname{norm}(\mathrm{rq}) / \mathrm{nq}=0.0000$

$\operatorname{norm}(\mathrm{rv}) / \mathrm{nv}=0.0000$

$\begin{array}{ll}\operatorname{norm}(\mathrm{rsp}) / \mathrm{npu}= & 0.0000 \\ \operatorname{norm}(\mathrm{rsq}) / \mathrm{nqu} & =0.0000\end{array}$

norm $(\mathrm{rsv}) / \mathrm{nvu}=0.0000$

$\operatorname{norm}(\mathrm{rr}) / \mathrm{nc}=0.0003$

norm $(\mathrm{rs}) / \mathrm{nc}=0.0005$

$\underset{\sim \sim \sim \sim \sim \sim \sim}{\text { Variáveis Livres }}$

$\begin{array}{lll}\mathrm{yp}=0.5009 & 0.5123 & 0.4943 \\ \mathrm{yq}=0.0078 & 0.0075 & 0.0085\end{array}$

$\begin{array}{lll}\mathrm{yq}=0.0078 & 0.0075 & 0.0085 \\ \mathrm{yv}=0.0023 & 0.0018 & 0.0003\end{array}$

Parâmetros

$\mathrm{mi}=0.0012$

$\begin{array}{lll}\text { beta } & =0.1548 \\ \text { erro } & =0.0007\end{array}$

gamarel $=0.0007$

Resultados

Função Objetivo $=0.379$

iterações $=5$

tempo $=0.015$ 
BAR3 -- Esquema L-B

\begin{tabular}{|c|c|c|c|}
\hline \multicolumn{3}{|c|}{$\begin{array}{l}\text { Carga Ativa }=1.500000 \\
\text { Carga Reativa }=0.299700\end{array}$} & $\begin{array}{l}\text { Capacidade }=3.000000 \\
\text { Capacidade }=0.599400\end{array}$ \\
\hline \multicolumn{4}{|c|}{$\begin{array}{l}\text { Limites de tensão (L-B) } \\
\text { vmin }=0.96000 \\
\text { vmax }=1.04000\end{array}$} \\
\hline \multicolumn{4}{|c|}{ 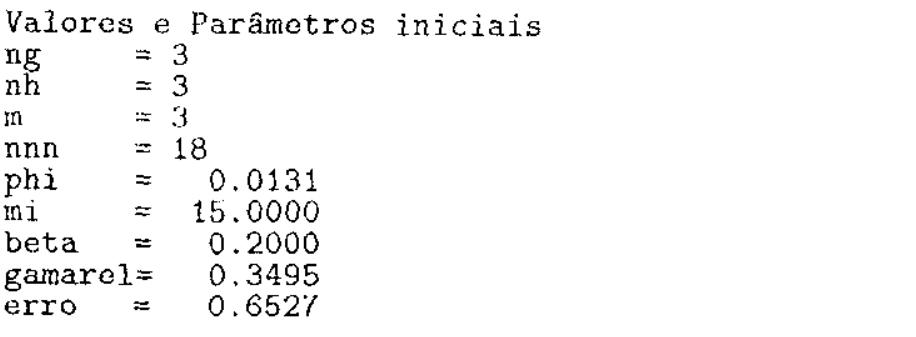 } \\
\hline \multicolumn{4}{|c|}{ 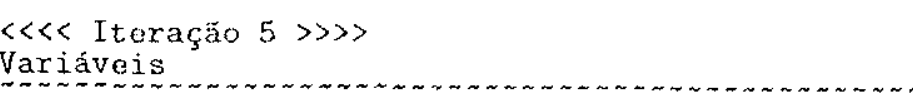 } \\
\hline $\begin{array}{l}\mathrm{p}= \\
\mathrm{q}= \\
\mathrm{v}= \\
\mathrm{r}= \\
\mathrm{s}= \\
\mathrm{sp}= \\
\mathrm{sq}= \\
\mathrm{sv}= \\
\mathrm{zp}= \\
\mathrm{zq}= \\
\mathrm{zv}= \\
\mathrm{wp}= \\
\mathrm{wq}= \\
\mathrm{w}=\end{array}$ & $\begin{array}{l}=0.5004 \\
=0.1152 \\
=0.0911 \\
=0.7054 \\
=0.7178 \\
=0.4996 \\
=0.0646 \\
=0.0034 \\
=0.0140 \\
=0.0183 \\
=0.0036 \\
=0.0232 \\
=0.0270\end{array}$ & $\begin{array}{l}0.5137 \\
0.1210 \\
0.0850 \\
0.7457 \\
0.6712 \\
0.4863 \\
0.0788 \\
0.0750 \\
0.0037 \\
0.0139 \\
0.0198 \\
0.0033 \\
0.0236 \\
0.0243\end{array}$ & $\begin{array}{l}0.4933 \\
0.1388 \\
0.0670 \\
0.7973 \\
0.5942 \\
0.5067 \\
0.0610 \\
0.0930 \\
0.0034 \\
0.0128 \\
0.0268 \\
0.0036 \\
0.0273 \\
0.0184\end{array}$ \\
\hline \multicolumn{4}{|c|}{ Direções } \\
\hline $\begin{array}{l}\text { dp } \\
d q \\
d v \\
d r \\
d s \\
d s p \\
d s q \\
d s v \\
d z p \\
d z q \\
d z v \\
d w p \\
d w q \\
d w v\end{array}$ & $\begin{array}{l}=0.0035 \\
=0.0029 \\
=0.0014 \\
=0.0016 \\
=-0.0020 \\
=-0.0035 \\
=-0.0029 \\
=-0.0014 \\
=-0.0100 \\
=-0.0444 \\
=-0.0550 \\
=-0.0109 \\
=-0.0766 \\
=-0.0815\end{array}$ & $\begin{array}{r}-0.0123 \\
0.0015 \\
0.0011 \\
0.0017 \\
-0.0027 \\
0.0123 \\
-0.0015 \\
-0.0011 \\
-0.0111 \\
-0.0415 \\
-0.0599 \\
-0.0093 \\
-0.0771 \\
-0.0734\end{array}$ & $\begin{array}{r}0.0091 \\
-0.0008 \\
-0.0006 \\
0.0021 \\
-0.0056 \\
-0.0091 \\
0.0008 \\
0.0006 \\
-0.0098 \\
-0.0370 \\
-0.0888 \\
-0.0110 \\
-0.0908 \\
-0.0531\end{array}$ \\
\hline \multicolumn{4}{|c|}{ Residuos } \\
\hline 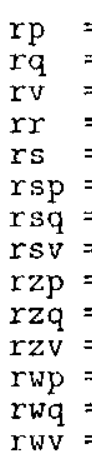 & $\begin{array}{l}=-0.0000 \\
=0.0000 \\
=-0.0000 \\
=-0.0003 \\
=0.0007 \\
=0.0000 \\
=0.0000 \\
=0.0000 \\
=-0.0049 \\
=-0.0048 \\
=-0.0048 \\
=-0.0056 \\
=-0.0070 \\
=-0.0059\end{array}$ & $\begin{array}{r}-0.0000 \\
0.0000 \\
-0.0000 \\
-0.0001 \\
0.0004 \\
0.0000 \\
0.0000 \\
0.0000 \\
-0.0060 \\
-0.0049 \\
-0.0049 \\
-0.0043 \\
-0.0063 \\
-0.0057\end{array}$ & $\begin{array}{r}0.0000 \\
-0.0001 \\
-0.0000 \\
0.0003 \\
-0.0011 \\
-0.0000 \\
-0.0000 \\
0.0000 \\
-0.0046 \\
-0.0052 \\
-0.0061 \\
-0.0058 \\
-0.0054 \\
-0.0049\end{array}$ \\
\hline
\end{tabular}


Normas

$\operatorname{norm}(\mathrm{rp}) / \mathrm{np}=0.0000$

$\operatorname{norm}(\mathrm{rq}) / \mathrm{nq}=0.0000$

$\operatorname{norm}(\mathrm{rv}) / \mathrm{nv}=0.0000$

$\operatorname{norm}(\mathrm{rsp}) / \mathrm{npu}=0.0000$

$\operatorname{norm}(\mathrm{rsq}) / \mathrm{nqu}=0.0000$

$\begin{array}{ll}\operatorname{norm}(\mathrm{rsv}) / \mathrm{nvu} & =0.0000 \\ \operatorname{norm}(\mathrm{rr}) / \mathrm{nc} & =0.0002\end{array}$

$\operatorname{norm}(\mathrm{rs}) / \mathrm{nc}=0.0005$

$\underset{\sim}{\text { Variáveis Livres }}$

$y \mathrm{p}=0.5006 \quad 0.5133 \quad 0.4936$

$\mathrm{yq}=\begin{array}{lll}0.0092 & 0.0097 & 0.0146\end{array}$

Para âmetros

$\mathrm{mi}=0.0017$

$=0.1548$

gamarel $=0.0010$

Resultados

Função Objetivo $=0.379$

iterações $=5$

tempo $=0.015$

flops $=14053$ 


\section{AR3 - Esquema $\mathrm{I}, \mathrm{C}$}

\begin{tabular}{|c|c|c|c|}
\hline \multicolumn{2}{|c|}{ Carga Átjva } & \multicolumn{2}{|c|}{$=1.500000 \quad$ Capacidade $=3.000000$} \\
\hline \multicolumn{4}{|c|}{$\begin{array}{l}\text { Limites de tensão }(l-C) \\
\text { vmin }=0.98000 \\
\operatorname{vmax}=1.02000\end{array}$} \\
\hline \multicolumn{4}{|c|}{ 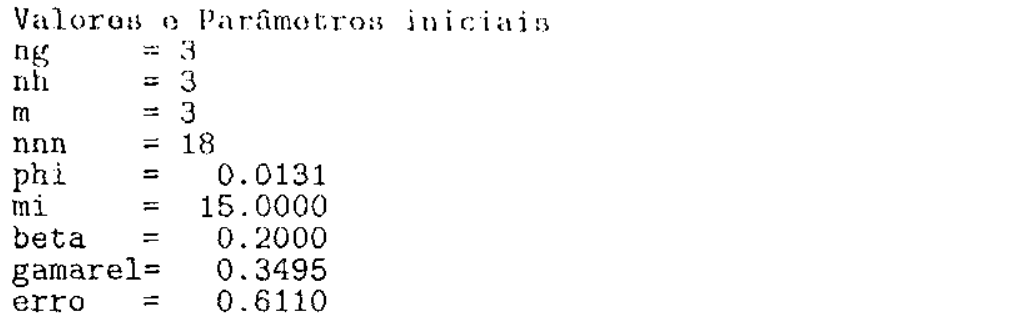 } \\
\hline \multicolumn{4}{|c|}{$\begin{array}{l}\text { 《<< Iteração } 6 \gg \gg \\
\text { Variaveis }\end{array}$} \\
\hline $\begin{array}{l}\mathrm{p}= \\
\mathrm{q}= \\
\mathrm{v}= \\
\mathrm{r}= \\
\mathrm{s}= \\
\mathrm{sp}= \\
\mathrm{sq}= \\
\mathrm{sv}= \\
\mathrm{zp}= \\
\mathrm{zq}= \\
\mathrm{zv}= \\
\mathrm{wp}= \\
\mathrm{wq}= \\
\mathrm{wv}=\end{array}$ & $\begin{array}{l}0.5008 \\
0.1085 \\
0.0487 \\
0.7078 \\
0.7130 \\
0.4992 \\
0.0913 \\
0.0313 \\
0.0011 \\
0.0049 \\
0.0107 \\
0.0011 \\
0.0064 \\
0.0193\end{array}$ & $\begin{array}{l}0.5119 \\
0.1183 \\
0.0435 \\
0.7484 \\
0.6663 \\
0.4881 \\
0.0815 \\
0.0365 \\
0.0011 \\
0.0046 \\
0.0122 \\
0.0011 \\
0.0069 \\
0.0159\end{array}$ & $\begin{array}{l}0.4947 \\
0.1488 \\
0.0299 \\
0.8015 \\
0.5899 \\
0.5053 \\
0.0510 \\
0.0501 \\
0.0011 \\
0.0038 \\
0.0191 \\
0.0011 \\
0.0103 \\
0.0108\end{array}$ \\
\hline \multicolumn{4}{|c|}{ 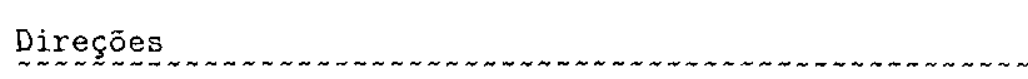 } \\
\hline $\begin{array}{l}d p \\
d q \\
d v \\
d r \\
d s \\
d s p \\
d s q \\
d s v \\
d z p \\
d z q \\
d z v \\
d w p \\
d w q \\
d w v\end{array}$ & $\begin{array}{l}=0.0018 \\
=0.0017 \\
=0.0008 \\
=0.0102 \\
=-0.0092 \\
=-0.0018 \\
=-0.0017 \\
=-0.0008 \\
=-0.0034 \\
=-0.0154 \\
=-0.0038 \\
=-0.0215 \\
=-0.0812\end{array}$ & $\begin{array}{r}-0.0051 \\
0.0005 \\
0.0006 \\
0.0095 \\
-0.0100 \\
0.0051 \\
-0.0005 \\
-0.0006 \\
-0.0040 \\
-0.0144 \\
-0.0398 \\
-0.0032 \\
-0.0238 \\
-0.0518\end{array}$ & $\begin{array}{r}0.0034 \\
-0.0008 \\
-0.0003 \\
0.0089 \\
-0.0120 \\
-0.0034 \\
0.0008 \\
0.0003 \\
-0.0033 \\
-0.0118 \\
-0.0696 \\
-0.0039 \\
-0.0378 \\
-0.0335\end{array}$ \\
\hline \multicolumn{4}{|c|}{ Residuos } \\
\hline $\begin{array}{l}r p \\
r q \\
r v \\
r r \\
r s \\
r s p \\
r s q \\
r s v \\
r z p \\
r z q \\
r z v \\
r w p \\
r w q \\
r w v\end{array}$ & $\begin{array}{l}=-0.0001 \\
=0.0000 \\
=-0.0002 \\
=-0.0000 \\
=0.0001 \\
=0.0000 \\
=0.0000 \\
=-0.0000 \\
=-0.0017 \\
=-0.0016 \\
=-0.0017 \\
=-0.0020 \\
=-0.0020\end{array}$ & $\begin{array}{r}-0.0000 \\
0.0000 \\
-0.0002 \\
-0.0000 \\
0.0001 \\
0.0000 \\
0.0000 \\
0.0000 \\
-0.0021 \\
-0.0017 \\
-0.0017 \\
-0.0015 \\
-0.0020 \\
-0.0020\end{array}$ & $\begin{array}{r}0.0001 \\
-0.0001 \\
-0.0002 \\
0.0001 \\
-0.0002 \\
0.0000 \\
0.0000 \\
0.0000 \\
-0.0016 \\
-0.0018 \\
-0.0021 \\
-0.0020 \\
-0.0019 \\
-0.0017\end{array}$ \\
\hline
\end{tabular}


Normas

$\operatorname{norm}(\mathrm{rp}) / \mathrm{np}=0.0000$

$\operatorname{norm}(\mathrm{rq}) / \mathrm{nq}=0.0000$

$\operatorname{norm}(\mathrm{rv}) / \mathrm{nv}=0.0001$

(rsq)/nqu =

norm (rsq) $/$ nqu $=0.0000$

norm $(r s v) / n v u=0.0000$

$\begin{array}{ll}\operatorname{norm}(\mathrm{rr}) / \mathrm{nc} & =: \\ \operatorname{norm}(\mathrm{rs}) / \mathrm{nc} & =0.0000\end{array}$

Variáveis Livres

$\begin{array}{lll}\text { yp }=0.5008 & 0.5119 & 0.4948 \\ \text { yq }=0.0016 & 0.0023 & 0.0065\end{array}$

$\begin{array}{llll}\mathrm{yv}= & =0.0086 & 0.0038 & -0.0083\end{array}$

Parâmetros

$\mathrm{mi}=0.0006$

beta $=0.1470$

gamarel $=0.0003$

Resultados

Função Objetivo $=0.379$

iterações $=6$

flops $=16812$ 


\section{BAR3 - Esquema L-D}

\begin{tabular}{|c|c|c|c|}
\hline \multicolumn{3}{|c|}{$\begin{array}{l}\text { Carga Ativa }=1.500000 \\
\text { Carga Reativa }=0.299700\end{array}$} & 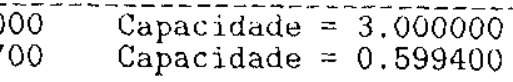 \\
\hline \multicolumn{4}{|c|}{$\begin{array}{l}\text { Limites de tensão (L-D) } \\
\text { vmin }=0.99999\end{array}$} \\
\hline \multicolumn{4}{|c|}{$\begin{array}{ll}\mathrm{ng} & =3 \\
\mathrm{nh} & =3 \\
\mathrm{~m} & =3 \\
\mathrm{~nm} & =18 \\
\mathrm{phi} & =0.0131 \\
\mathrm{mi} & =15.0000 \\
\text { beta } & =0.2000 \\
\text { gamarel } & =0.3495 \\
\text { erro } & =0.5706\end{array}$} \\
\hline \multicolumn{4}{|c|}{$\begin{array}{l}\ll<<\text { Iteraçầ } 6 \gg \gg> \\
\text { Variaveis }\end{array}$} \\
\hline $\begin{array}{l}\mathrm{p}= \\
\mathrm{q}= \\
\mathrm{v}= \\
\mathrm{r}= \\
\mathrm{s}= \\
\mathrm{sp}= \\
\mathrm{sq}= \\
\mathrm{sv}= \\
\mathrm{zp}= \\
\mathrm{zq}= \\
\mathrm{zv}= \\
\mathrm{wp}= \\
\mathrm{wq}= \\
\mathrm{wV}=\end{array}$ & $\begin{aligned}= & 0.4980 \\
= & 0.0820 \\
= & 0.0000 \\
= & 0.6583 \\
= & 0.7528 \\
= & 0.5020 \\
= & 0.1178 \\
= & 0.0000 \\
= & 0.0008 \\
= & 0.0048 \\
= & 18.4037 \\
= & 0.0009 \\
= & 0.0035 \\
= & 18.4580\end{aligned}$ & $\begin{array}{r}0.5180 \\
0.1125 \\
0.0000 \\
0.7038 \\
0.7104 \\
0.4820 \\
0.0873 \\
0.0000 \\
0.0009 \\
0.0036 \\
18.4154 \\
0.0007 \\
0.0047 \\
18.4570\end{array}$ & $\begin{array}{r}0.4914 \\
0.1801 \\
0.0000 \\
0.7659 \\
0.6429 \\
0.5086 \\
0.0197 \\
0.0000 \\
0.0008 \\
0.0021 \\
18.4792 \\
0.0009 \\
0.0466 \\
18.3886\end{array}$ \\
\hline \multicolumn{4}{|c|}{ Direcões } \\
\hline $\begin{array}{l}d p \\
d q \\
d v \\
d r \\
d s \\
d s p \\
d s q \\
d s v \\
d z p \\
d z q \\
d z v \\
d w p \\
d w q \\
d w v\end{array}$ & $\begin{array}{l}=0.0077 \\
=0.0006 \\
=-0.0000 \\
=-0.0002 \\
=-0.0002 \\
=-0.0075 \\
=-0.0006 \\
=-0.0000 \\
=-0.0032 \\
=-0.0199 \\
=1.4593 \\
=-0.0034 \\
=-0.0158 \\
=1.3203\end{array}$ & $\begin{array}{r}-0.0172 \\
0.0002 \\
-0.0000 \\
0.0001 \\
-0.0004 \\
0.0174 \\
-0.0002 \\
-0.0000 \\
-0.0034 \\
-0.0147 \\
1.4777 \\
-0.0030 \\
-0.0222 \\
1.3856\end{array}$ & $\begin{array}{r}0.0103 \\
0.0041 \\
-0.0000 \\
0.0029 \\
-0.0038 \\
-0.0101 \\
-0.0041 \\
-0.0000 \\
-0.0031 \\
-0.0082 \\
1.2580 \\
-0.0034 \\
-0.1231 \\
1.4856\end{array}$ \\
\hline \multicolumn{4}{|c|}{$\operatorname{Res}_{\sim \sim \sim \sim \sim \sim \sim \sim \sim \sim \sim \sim \sim \sim \sim \sim \sim \sim}^{\text {idduos }}$} \\
\hline $\begin{array}{l}r p \\
r q \\
r v \\
r r \\
r s \\
r s p \\
r s q \\
r s v \\
r z p \\
r z q \\
r z v \\
r w p \\
r w q \\
r w v\end{array}$ & $\begin{array}{l}=0.0000 \\
=-0.0000 \\
=-0.0000 \\
=-0.0008 \\
=0.0007 \\
=0.0000 \\
=0.0000 \\
=0.0000 \\
=-0.0015 \\
=-0.0016 \\
=-0.0006 \\
=-0.0017 \\
=-0.0019 \\
=-0.0001\end{array}$ & $\begin{array}{r}0.0000 \\
0.0000 \\
-0.0000 \\
-0.0008 \\
0.0008 \\
-0.0000 \\
0.0000 \\
0.0000 \\
-0.0019 \\
-0.0016 \\
-0.0003 \\
-0.0013 \\
-0.0019 \\
-0.0004\end{array}$ & $\begin{array}{r}-0.0000 \\
-0.0001 \\
-0.0000 \\
0.0014 \\
-0.0017 \\
0.0000 \\
-0.0000 \\
0.0000 \\
-0.0015 \\
-0.0014 \\
-0.0002 \\
-0.0018 \\
-0.0036 \\
-0.0006\end{array}$ \\
\hline
\end{tabular}


Normas

$\operatorname{norm}(\mathrm{rp}) / \mathrm{np}=0.0000$

$\operatorname{norm}(\mathrm{rq}) / \mathrm{nq}=0.0000$

$\operatorname{norm}(\mathrm{rv}) / \mathrm{nv}=0.0000$

norm $(\mathrm{rsq}) / \mathrm{nqu}=0.0000$

$\operatorname{norm}(\mathrm{rsv}) / \mathrm{nvu}=0.0000$

$\begin{array}{ll}\operatorname{norm}(\mathrm{rr}) / \mathrm{nc} & =0.0007 \\ \text { norm } & \mathrm{rs}) / \mathrm{nc}\end{array}$

Variáveis Livres

yp $=0.4981 \quad 0.5178 \quad 0.4915$

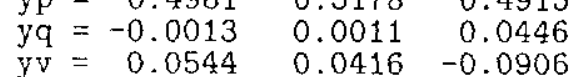

Parâmetros

$\mathrm{mi}=0.0004$

$\begin{array}{ll}\text { beta } & =0.1470 \\ \text { erro } & =0.0007\end{array}$

gamarel $=0.0002$

Resultados

Função Objetivo $=0.379$

iterações $=6$

tempo $=0.016$

flops $=16812$ 
IEEE30 - Esquema L-A

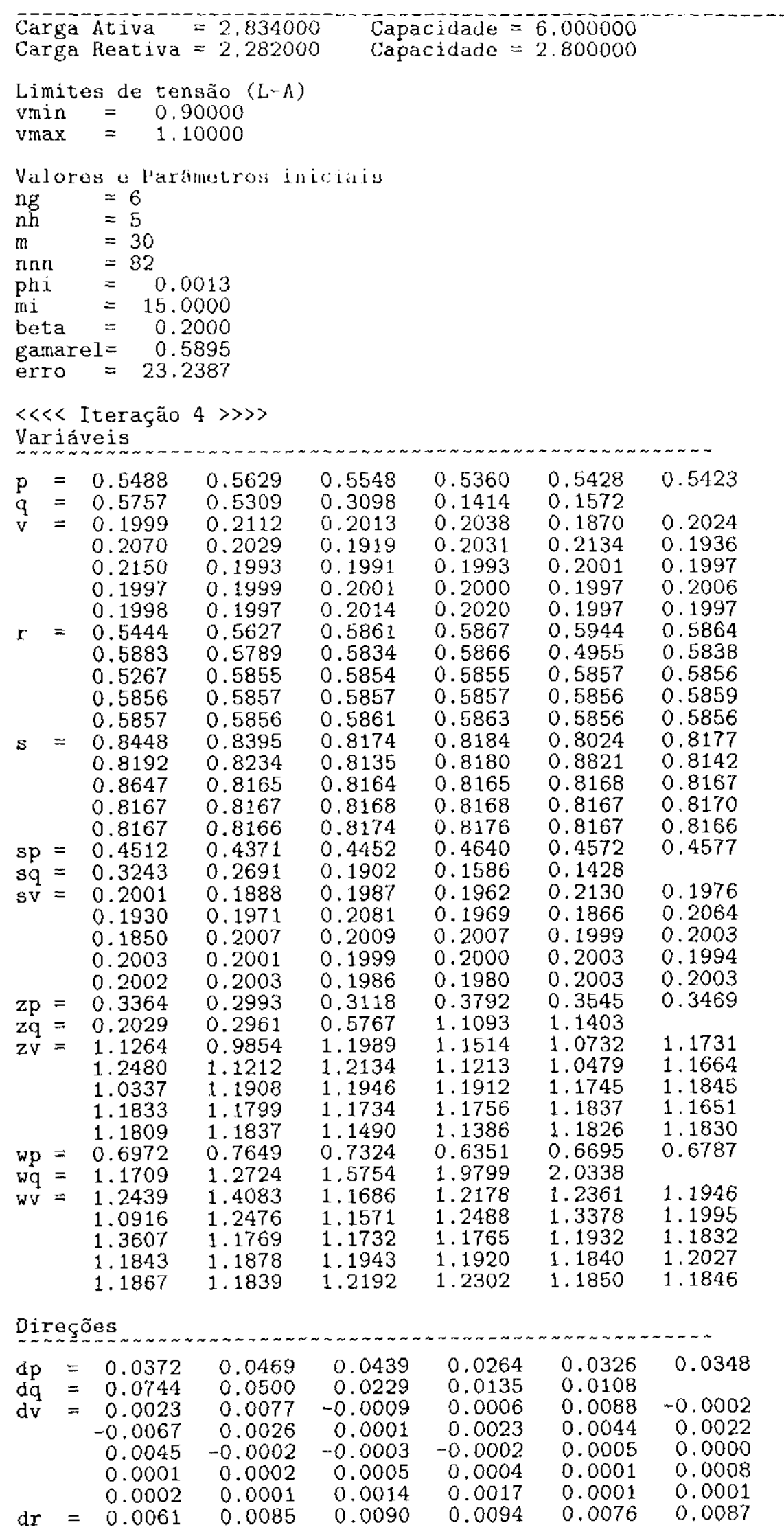


10

\begin{tabular}{|c|c|c|c|c|c|c|c|}
\hline & & & & & & & \\
\hline & & 0.0053 & 0.0085 & 0.0090 & 0.0095 & 0.0058 & 0.0097 \\
\hline & & & 0.0089 & 0.0089 & 0.0089 & 0.0090 & 0.0089 \\
\hline & & & 0.0089 & 0.0090 & 0.0090 & 0.0089 & 0090 \\
\hline & & & 0.0087 & 0.0090 & 0.0092 & 0.0086 & 0.0086 \\
\hline $\mathbf{d s}$ & $=$ & -0.0032 & -0.0017 & -0.0076 & -0.0069 & & \\
\hline & & -0.0 & -0.0049 & -0.0070 & -0. & 014 & \\
\hline & & & & -0.0071 & & & \\
\hline & & & -0. & -0.0066 & -0.0067 & -0.0068 & \\
\hline & & & -0.0065 & -0.0060 & -0.0061 & -0.0065 & 065 \\
\hline & & -0.037 & -0.0469 & -0.0439 & -0.0264 & -0.0326 & \\
\hline & & -0.0 & $\begin{array}{l}-0.0 \\
-0.0\end{array}$ & $\begin{array}{r}-0.0229 \\
0.0009\end{array}$ & $\begin{array}{l}-0.0 \\
-0.0\end{array}$ & $\begin{array}{l}-0.0108 \\
-0.0088\end{array}$ & 0.0002 \\
\hline & & 0.0 & -0.0 & -0.0001 & -0.0 & -0.0044 & \\
\hline & & -0 . & & 0 & & & \\
\hline & & -0. & -0 . & -0 . & -0 . & -0 & \\
\hline & & -0.0 & -0. & -0 & -0. & -0 . & 001 \\
\hline & & -1.8 & -1. & -1. & -1 . & -1 & 3895 \\
\hline & & $\begin{array}{l}-2.0 \\
-4.0\end{array}$ & $\begin{array}{l}-2 . \\
-4\end{array}$ & $\begin{array}{l}-3.3884 \\
-4.1783\end{array}$ & $\begin{array}{l}-5 . \\
-4 .\end{array}$ & $\begin{array}{l}-5 \\
-4\end{array}$ & -4.0999 \\
\hline & & -3.8 & -4 & -4.1514 & & & \\
\hline & & & & -4. & & & \\
\hline & & & -4 & -4.1 & -4 & & \\
\hline & & & -4 & -4.16 & -4.1 & -4.1670 & -4 \\
\hline & $=$ & -1.9403 & -1 & -1 & -1 & -1 & \\
\hline & & -2 . & -2 . & -3. & -5 & & \\
\hline & & -4 & -4 & -4.1 & -4 & -3. & -4.2339 \\
\hline & & & & & & & \\
\hline & & & & & & & \\
\hline & & & & & & & \\
\hline & & & & -4 & -4 & -4 & \\
\hline \multicolumn{8}{|c|}{ ardu } \\
\hline & & & & & & & \\
\hline & & $\begin{array}{r}0.000 \\
-0.368\end{array}$ & $\begin{array}{l}-0.0 \\
-0.0\end{array}$ & $\begin{array}{l}0.26 \\
0.53\end{array}$ & $\begin{array}{r}0 . \\
-0\end{array}$ & $\begin{array}{r}-0 \\
0\end{array}$ & \\
\hline & & -0.8 & -0 . & -0. & -0. & -0 & \\
\hline & & -0 . & -0 . & -0 & & -0 & \\
\hline & & & & & & -0 & \\
\hline & $=$ & 0 . & 0. & -0 . & 0 . & 0. & 0 \\
\hline & & & & & & & \\
\hline & & & -0 & & -0 . & & \\
\hline & & & -0. & -0 . & 0. & -0 & \\
\hline & & & -0 & & & -0 & -0 \\
\hline \multirow[t]{5}{*}{ rv } & & & -0 . & -0 . & -0 . & & \\
\hline & & & & -0. & -0 . & -0 & \\
\hline & & -0 & -0 . & -0 . & -0. & -0 & \\
\hline & & & & & & & \\
\hline & & & & & & -0 & \\
\hline & $=$ & -0 & & -0 & -0 & & \\
\hline & & & & & & & \\
\hline & & & & & & & \\
\hline & & & $\begin{array}{r}-0 . \\
0 .\end{array}$ & -0 & -0 . & -0 . & \\
\hline \multirow[t]{5}{*}{ rs } & & & & & $\begin{array}{r}0 . \\
-0 .\end{array}$ & & $\begin{array}{l}-0 . \\
-0\end{array}$ \\
\hline & & & & -0 & -0 . & & -0. \\
\hline & & & & & & & \\
\hline & & & & & & & \\
\hline & & & & & & & \\
\hline & & -0 & & & & & \\
\hline & & & & & & & \\
\hline \multirow[t]{5}{*}{$x \mathrm{sv}^{3}$} & & & & & & & \\
\hline & & & & & & & \\
\hline & & & & & & & \\
\hline & & & & & & & \\
\hline & & & & & & & \\
\hline & & & & & & & -0.8933 \\
\hline & & & & & -0.6 & -0.7 & \\
\hline & & & -0.8046 & -0.8 & -0.8 & -0.8 & -0.8 \\
\hline & & & & & & & \\
\hline & & $\begin{array}{l}-0 \\
-0\end{array}$ & $\begin{array}{l}-0.83 \\
-0.83\end{array}$ & $\begin{array}{l}-0.83 \\
-0.83\end{array}$ & $\begin{array}{l}-0 . \\
-0 .\end{array}$ & $\begin{array}{l}-0 . \\
-0\end{array}$ & $\begin{array}{l}-0.8314 \\
-0.8288\end{array}$ \\
\hline & & & -0. & $\begin{array}{l}-0.8 \\
-0.8\end{array}$ & $\begin{array}{l}-0 . \\
-0 .\end{array}$ & $\begin{array}{l}-0 . \\
-0 .\end{array}$ & $\begin{array}{l}-0 \\
-0\end{array}$ \\
\hline & & & 1 . & -1.04 & -1.0055 & -1.0213 & -1.032 \\
\hline
\end{tabular}




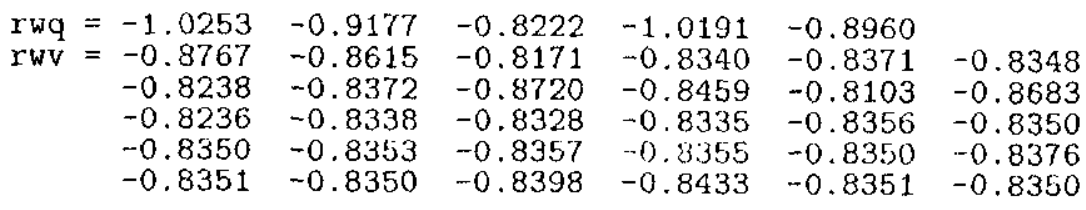

Variádeis Livres

\begin{tabular}{|c|c|c|c|c|c|}
\hline $\begin{array}{l}\mathrm{yp}= \\
\mathrm{yq}= \\
\mathrm{yv}=\end{array}$ & $\begin{array}{r}0.9096 \\
0.9680 \\
0.1175 \\
0.0215 \\
0.2899 \\
-0.0147 \\
0.0209 \\
0.0001\end{array}$ & $\begin{array}{r}1.0285 \\
0.9763 \\
0.4229 \\
-0.1564 \\
0.0331 \\
0.0187 \\
0.0164 \\
0.0702\end{array}$ & $\begin{array}{r}0.9753 \\
0.9986 \\
-0.0302 \\
0.1264 \\
0.3270 \\
-0.0013 \\
0.0003 \\
0.0917\end{array}$ & $\begin{array}{r}0.7919 \\
0.8706 \\
0.0665 \\
-0.0563 \\
-0.0139 \\
0.0010 \\
0.0376 \\
0.0024\end{array}$ & $\begin{array}{r}0.8577 \\
0.8935 \\
0.1629 \\
0.1275 \\
-0.0214 \\
0.0079 \\
0.0058 \\
0.0017\end{array}$ \\
\hline
\end{tabular}

$\underset{\sim \sim \sim \sim \sim \sim \sim}{\text { Parametros }}$

$\begin{array}{ll}\mathrm{mi} & =0.1600 \\ \text { beta } & =0.2000\end{array}$

exro $=0.0970$

gamarel $=0.0853$

Resultados

Função Objetivo $=0.901$

iterações $=4$

$\begin{array}{ll}\text { tempo } & =0.047 \\ \text { flops } & =98906\end{array}$ 
IEEE30 - Esquema L-B

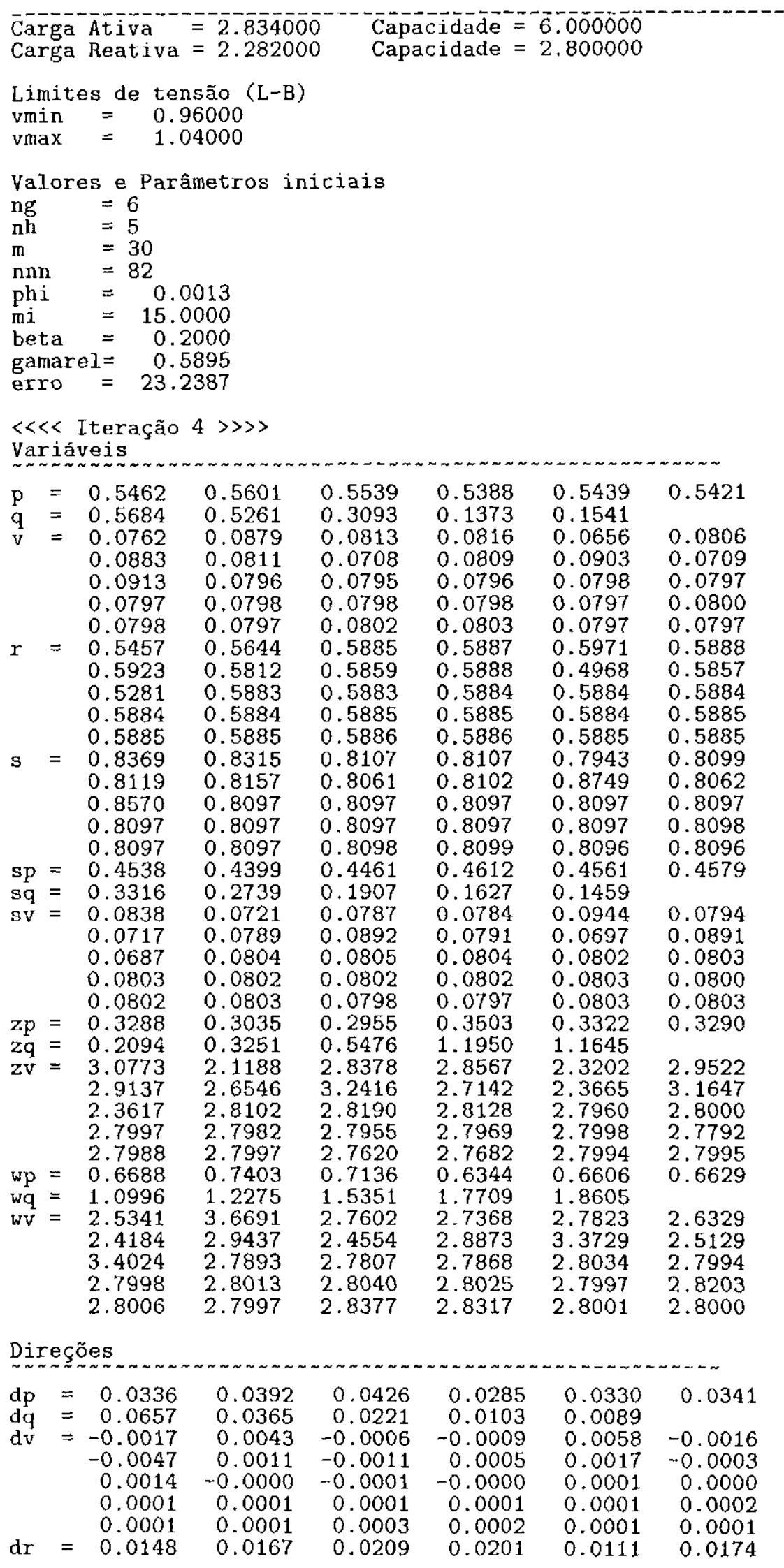




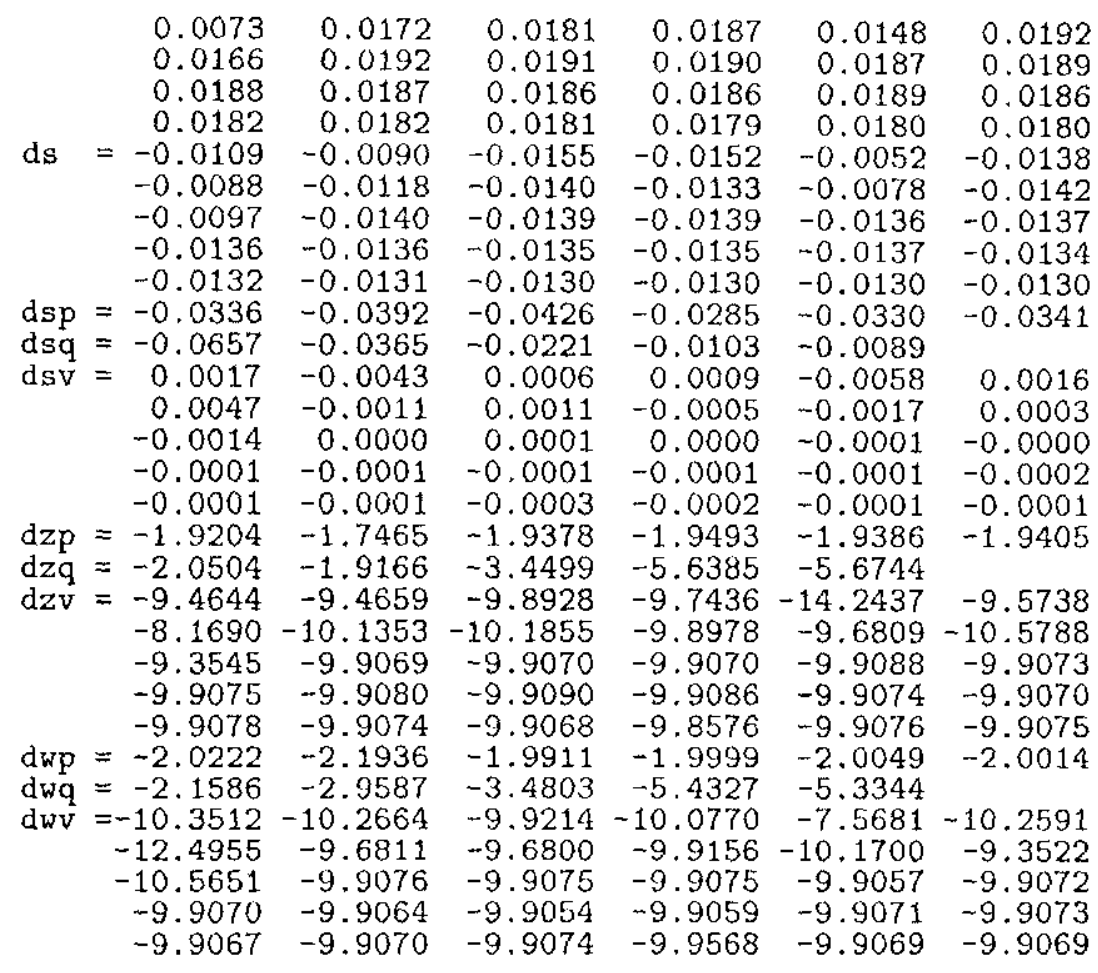
Residuos

$\mathrm{rp}=\begin{array}{llllll}0.0010 & -0.0011 & 0.2480 & 0.0837 & -0.0005 & 0.3917\end{array}$ $\begin{array}{llllll}-0.3634 & -0.0004 & 0.5389 & -0.0659 & -0.0002 & 0.4607\end{array}$ $\begin{array}{llllll}-0.0002 & -0.0688 & -0.0949 & -0.0434 & -0.0878 & -0.0322\end{array}$ $\begin{array}{llllll}-0.0949 & -0.0210 & -0.1722 & 0.0020 & -0.0320 & -0.0876\end{array}$

$r q=\begin{array}{rrrrrr}0.0007 & -0.0350 & -0.0009 & 0.0425 & -0.0237 & -0.1058 \\ 0.3179 & 0.0005 & -0.0834 & 0.1859 & 0.0003 & 0.1360\end{array}$ $\begin{array}{llllll}-0.1803 & 0.0008 & 0.1873 & 0.1612 & 0.0004 & 0.3001\end{array}$ $\begin{array}{llllll}0.0005 & -0.0300 & -0.0505 & -0.0356 & -0.0527 & -0.0094\end{array}$ $\begin{array}{llllll}-0.0339 & -0.0048 & -0.1062 & 0.0037 & -0.0160 & -0.0250\end{array}$

$\mathrm{v}=-\begin{array}{llllll}0.00004 & -0.0004 & -0.0007 & -0.0006 & -0.0002 & -0.0005\end{array}$ $\begin{array}{llllll}-0.0002 & -0.0005 & -0.0005 & -0.0005 & -0.0003 & -0.0006\end{array}$ $\begin{array}{llllll}-0.0004 & -0.0006 & -0.0006 & -0.0006 & -0.0005 & -0.0005\end{array}$ $\begin{array}{llllll}-0.0005 & -0.0005 & -0.0005 & -0.0005 & -0.0005 & -0.0005\end{array}$ $\begin{array}{lllllll}-0.0005 & -0.0005 & -0.0005 & -0.0005 & -0.0005 & -0.0005\end{array}$

$\mathrm{rr}=\begin{array}{lllllll}-0.0063 & 0.0506 & -0.0006 & 0.0009 & 0.0075 & -0.0040\end{array}$ $\begin{array}{llllll}-0.0451 & 0.0025 & -0.0013 & 0.0006 & 0.0018 & -0.0094\end{array}$ $\begin{array}{llllll}0.0103 & 0.0000 & 0.0000 & 0.0000 & -0.0001 & 0.0000\end{array}$ $\begin{array}{lllllll}-0.0000 & -0.0000 & -0.0001 & -0.0001 & -0.0000 & 0.0000\end{array}$ $\begin{array}{lllllll}-0.0000 & 0.0000 & 0.0000 & 0.0003 & -0.0000 & 0.0000\end{array}$

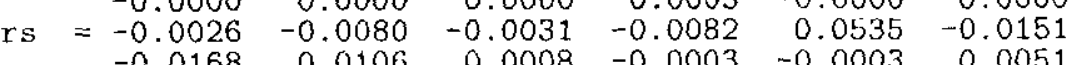
$\begin{array}{llllll}-0.0168 & 0.0106 & 0.0008 & -0.0003 & -0.0003 & 0.0051\end{array}$ $\begin{array}{llllll}-0.0040 & 0.0001 & 0.0001 & 0.0001 & 0.0002 & 0.0001\end{array}$ $\begin{array}{llllll}0.0001 & 0.0001 & 0.0002 & 0.0002 & 0.0001 & 0.0001 \\ 0.0001 & 0.0001 & 0.0001 & -0.0020 & 0.0001 & 0.0001\end{array}$

$r$ rp $=\begin{array}{rlllll}0.0001 & 0.0001 & 0.0001 & -0.0020 & 0.0001 & 0.0001\end{array}$

$\mathrm{rsq}=\begin{array}{lllll}0.0000 & 0.0000 & 0.0000 & 0.0000 & 0.0000\end{array}$

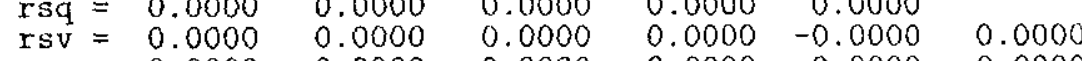
$\begin{array}{llllll}0.0000 & 0.0000 & 0.0000 & 0.0000 & 0.0000 & 0.0000 \\ 0.0000 & 0.0000 & 0.0000 & 0.0000 & 0.0000 & 0.0000\end{array}$ $\begin{array}{llllll}0.0000 & 0.0000 & 0.0000 & 0.0000 & 0.0000 & 0.0000\end{array}$ $\begin{array}{llllll}0.0000 & 0.0000 & 0.0000 & 0.0000 & 0.0000 & 0.0000 \\ 0.0000 & 0.0000 & 0.0000 & 0.0000 & 0.0000 & 0.0000\end{array}$ $r z p=\begin{array}{rrrrrr}0.9191 & -0.8404 & -0.9092 & -0.9381 & -0.9258 & -0.9192\end{array}$

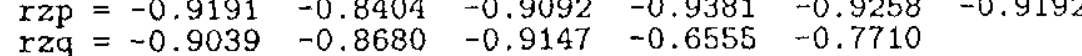

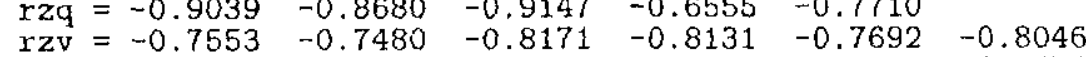
$\begin{array}{llllll}-0.8049 & -0.7990 & -0.7451 & -0.7902 & -0.8399 & -0.7574 \\ -0.8270 & -0.7990 & -0.7890 & -0.7890 & -0.7890 & -0.7890\end{array}$ $\begin{array}{llllll}-0.8270 & -0.7890 & -0.7890 & -0.7890 & -0.7890 & -0.7890 \\ -0.7890 & -0.7890 & -0.7890 & -0.7890 & -0.7890 & -0.7892\end{array}$ $\begin{array}{llllll}-0.7890 & -0.7890 & -0.7890 & -0.7890 & -0.7890 & -0.7892 \\ -0.7890 & -0.7890 & -0.7894 & -0.7881 & -0.7890 & -0.7890\end{array}$

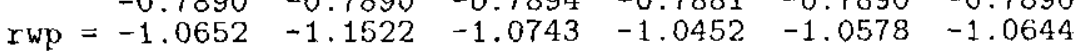


$\begin{array}{rllllll}r w q= & -1.0490 & -1.0534 & -0.8392 & -1.0046 & -0.8824 & \\ r w v= & -0.8315 & -0.8372 & -0.7680 & -0.7715 & -0.8112 & -0.7802 \\ & -0.7776 & -0.7863 & -0.8414 & -0.7950 & -0.7463 & -0.8269 \\ & -0.7574 & -0.7962 & -0.7961 & -0.7962 & -0.7962 & -0.7962 \\ & -0.7962 & -0.7962 & -0.7962 & -0.7962 & -0.7962 & -0.7959 \\ & -0.7962 & -0.7962 & -0.7957 & -0.7971 & -0.7962 & -0.7962\end{array}$

Normas

$\operatorname{norm}(\mathrm{rp}) / \mathrm{np}=0.0975$

$\operatorname{norm}(\mathrm{rq}) / \mathrm{nq}=0.0650$

norm(rv) $/ \mathrm{nv}=0.0005$

$\operatorname{norm}(\mathrm{rsp}) / \mathrm{npu}=0.0000$

$\operatorname{norm}(\mathrm{rsq}) / \mathrm{nqu}=0.0000$

norm $(\mathrm{rsv}) / \mathrm{nvu}=0.0000$

norm $(\mathrm{rs}) / \mathrm{nc}=0.0203$

Variáveis Livres

$\begin{array}{rrrrrrr}\mathrm{yp}= & 0.8862 & 0.9969 & 0.9719 & 0.8230 & 0.8724 & 0.8760 \\ \mathrm{yq}= & 0.8901 & 0.9024 & 0.9874 & 0.5759 & 0.6960 & \\ \mathrm{yv}=-0.5433 & 1.5504 & -0.0776 & -0.1199 & 0.4621 & \\ -0.3193 & -0.4953 & 0.2891 & -0.7862 & 0.1732 & \\ 1.0064 & -0.6518 & 1.0408 & -0.0209 & -0.0384 & \\ -0.0259 & 0.0074 & -0.0006 & 0.0001 & 0.0031 & \\ 0.0085 & 0.0056 & -0.0001 & 0.0412 & 0.0018 & \\ -0.0000 & 0.0757 & 0.0635 & 0.0007 & 0.0004 & \\ \text { Parâmetros } & & & & \end{array}$

$\underset{\sim \sim \sim \sim \sim}{\text { Parâmetros }}$

$\mathrm{mi}=0.1600$

beta $=0.2000$

gamarel $=0.0813$

Resultados

Função Objetivo= 0.899

iterações $=4$

$\begin{array}{ll}\text { tempo } & =0.047 \\ \text { flops } & =98594\end{array}$ 
IEEE30 - Esquema L-C

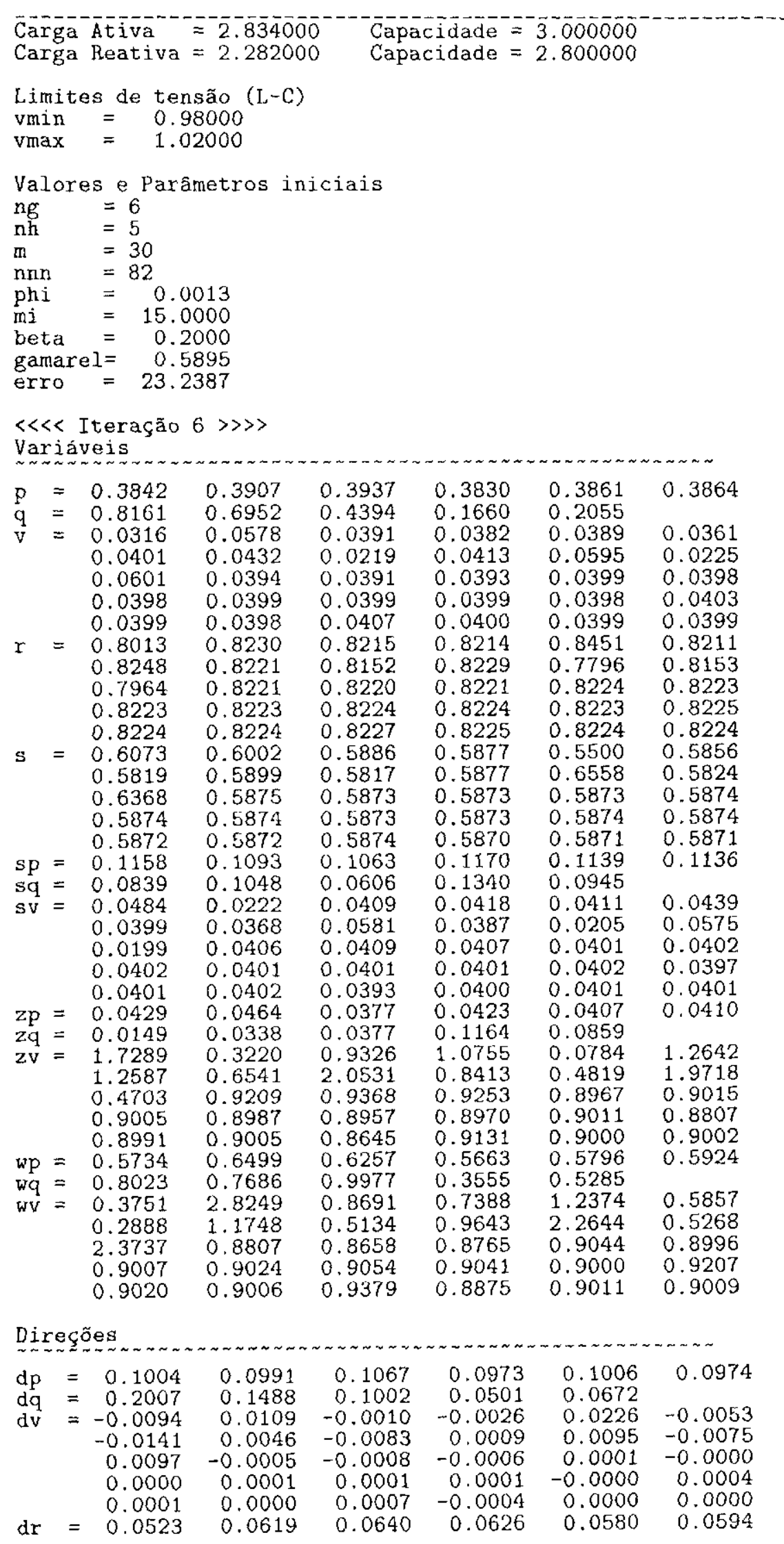




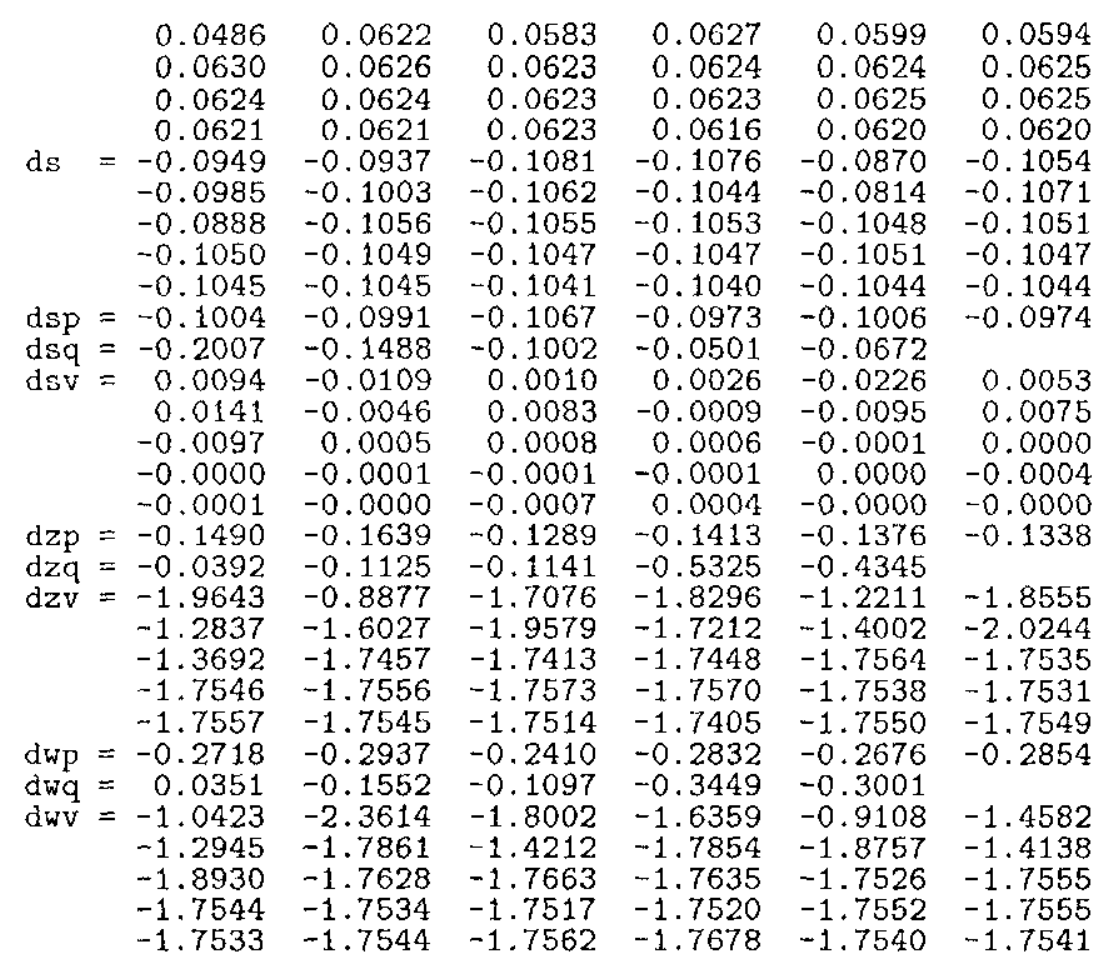

Residuos

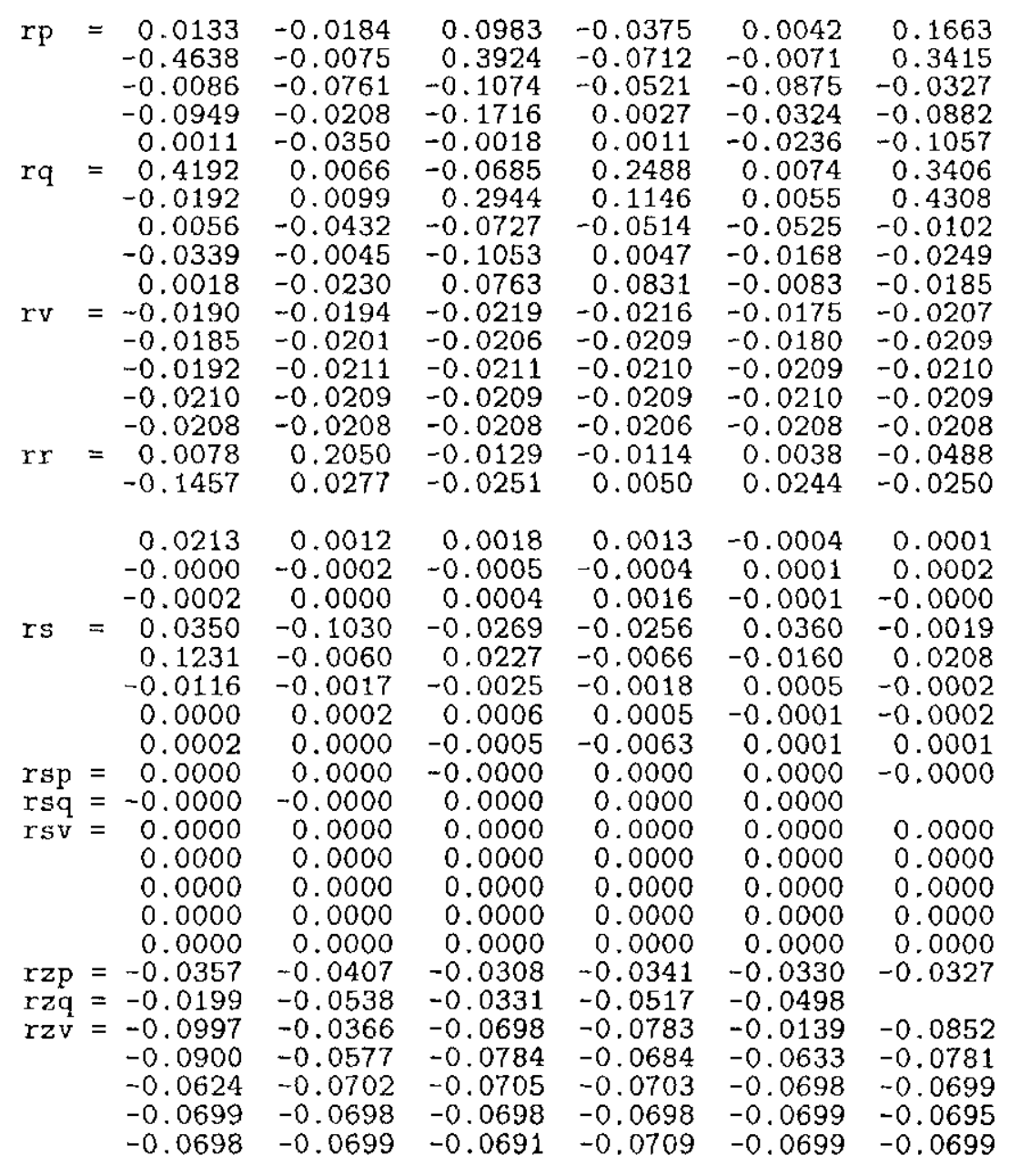




$\begin{array}{rllllll}\text { rwp }= & -0.1205 & -0.1301 & -0.1221 & -0.1200 & -0.1200 & -0.1222 \\ r w q= & -0.1499 & -0.1573 & -0.1194 & -0.0840 & -0.0872 & \\ r w v= & -0.0356 & -0.1130 & -0.0706 & -0.0615 & -0.0891 & -0.0519 \\ & -0.0266 & -0.0807 & -0.0648 & -0.0720 & -0.0806 & -0.0650 \\ & -0.0820 & -0.0702 & -0.0699 & -0.0701 & -0.0706 & -0.0705 \\ & -0.0705 & -0.0705 & -0.0706 & -0.0705 & -0.0705 & -0.0709 \\ & -0.0705 & -0.0705 & -0.0713 & -0.0694 & -0.0705 & -0.0705\end{array}$

Normas

$\operatorname{norm}(\mathrm{rp}) / \mathrm{np}=0.0814$

$\operatorname{norm}(\mathrm{rq}) / \mathrm{nq}=0.0834$

$\operatorname{norm}(\mathrm{rv}) / \mathrm{nv}=0.0179$

$\operatorname{norm}(\mathrm{rsq}) / \mathrm{nqu}=0.0000$

$\operatorname{norm}(\mathrm{rsv}) / \mathrm{nvu}=0.0000$

$\operatorname{norm}(\mathrm{rr}) / \mathrm{nc}=0.0762$

Variáveis Livres

\begin{tabular}{|c|c|c|c|c|c|}
\hline $\begin{array}{l}y p= \\
y q= \\
y v=\end{array}$ & $\begin{array}{r}=0.9147 \\
=0.7874 \\
-1.3538 \\
-0.6785 \\
1.7825 \\
-0.0488 \\
0.0098 \\
0.0000\end{array}$ & $\begin{array}{r}0.9942 \\
0.7347 \\
2.5029 \\
-0.9699 \\
-1.4450 \\
0.0076 \\
0.0072 \\
0.0734\end{array}$ & $\begin{array}{r}0.9818 \\
0.9599 \\
-0.0636 \\
0.5207 \\
1.9034 \\
-0.0019 \\
-0.0011 \\
-0.0256\end{array}$ & $\begin{array}{r}0.9070 \\
0.2391 \\
-0.3368 \\
-1.5396 \\
-0.0402 \\
0.0002 \\
0.0401 \\
0.0011\end{array}$ & $\begin{array}{r}0.9250 \\
0.4426 \\
1.1590 \\
0.1230 \\
-0.0710 \\
0.0036 \\
0.0029 \\
0.0008\end{array}$ \\
\hline
\end{tabular}

Parametros

$\begin{array}{ll}\mathrm{mi} & =0.0064 \\ \text { beta } & =0.2000\end{array}$

$=0.0834$

gamarel $=0.0196$

Resultados

Função Objetivo $=0.450$

iterações $=6$

empo $=0.078$

$=146187$ 


\section{IEEE30 - Esquema L-D}

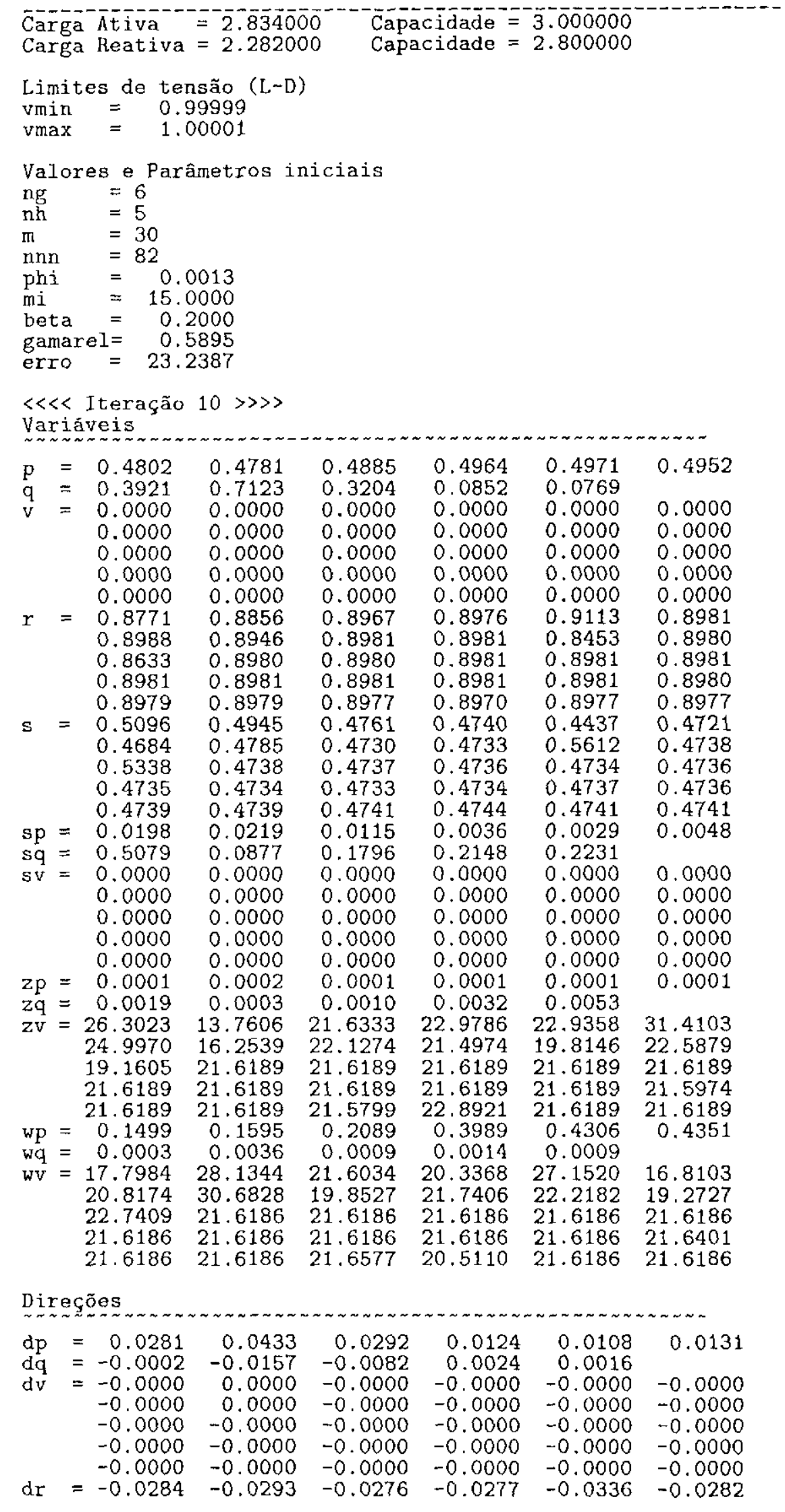




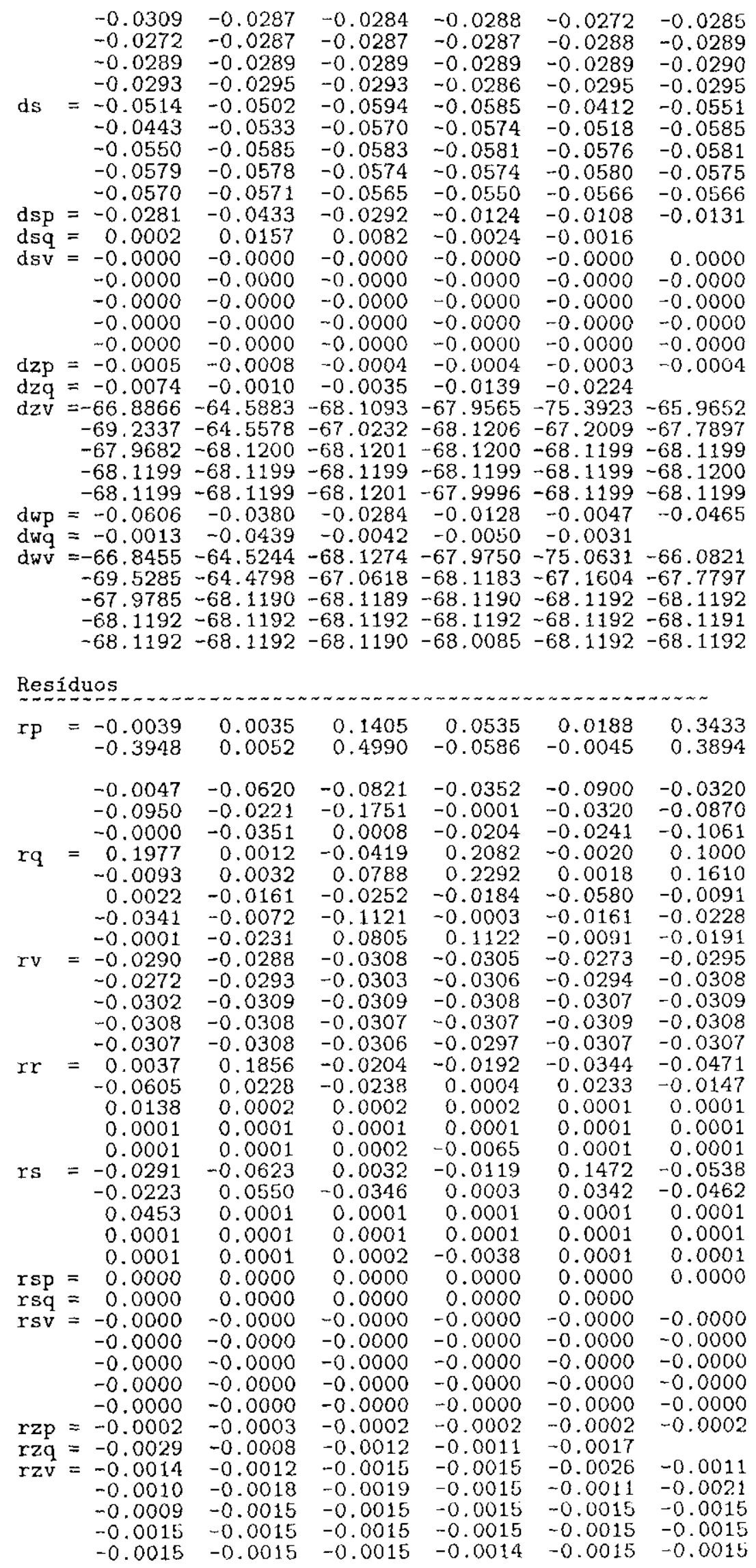




$\begin{array}{rllllll}\text { rwp }= & -0.0080 & -0.0102 & -0.0077 & -0.0052 & -0.0048 & -0.0068 \\ \text { rwq }= & -0.0007 & -0.0028 & -0.0007 & -0.0011 & -0.0007 & \\ \text { rwv }= & -0.0016 & -0.0017 & -0.0015 & -0.0016 & -0.0008 & -0.0019 \\ & -0.0021 & -0.0011 & -0.0011 & -0.0015 & -0.0019 & -0.0009 \\ & -0.0021 & -0.0015 & -0.0015 & -0.0015 & -0.0015 & -0.0015 \\ & -0.0015 & -0.0015 & -0.0015 & -0.0015 & -0.0015 & -0.0015 \\ & -0.0015 & -0.0015 & -0.0015 & -0.0016 & -0.0015 & -0.0015\end{array}$

Normas

$\operatorname{norm}(\mathrm{rp}) / \mathrm{np}=0.0910$

$\operatorname{norm}(\mathrm{rq}) / \mathrm{nq}=0.0497$

$\operatorname{norm}(\mathrm{rv}) / \mathrm{nv}=0.0255$

norm (rsq) $/ \mathrm{nqu}=0.0000$

norm (rsq)/nqu $=0.0000$

norm (rsv) $/ \mathrm{nvu}=0.0000$

norm(rs)/nc $=0.0611$

Variáveis Livres

$y p=\begin{array}{llllll}0.6300 & 0.6374 & 0.6973 & 0.8953 & 0.9277 & 0.9303\end{array}$

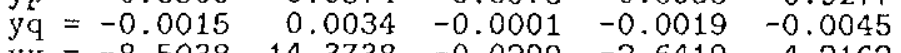

$\begin{array}{lllll}-8.5038 & 14.3738 & -0.0299 & -2.6419 & 4.2162\end{array}$

$\begin{array}{rrrrr}14.60036 & -3.3152 & 3.5804 & -0.0003 & -0.0004\end{array}$

$\begin{array}{lllll}-0.0003 & -0.0002 & -0.0002 & -0.0002 & -0.0002\end{array}$

$\begin{array}{rrrrr}-0.0002 & -0.0002 & -0.0002 & 0.0427 & -0.0002 \\ -0.0002 & 0.0779 & -2.3811 & -0.0002 & -0.0002\end{array}$

Parânetros

$\mathrm{mi}=0.0000$

$\begin{array}{ll}\text { beta } & =0.2000 \\ \text { erro } & =0.0910\end{array}$

erro $=0.0910$

gama $=0.0433$
gamarel $=0.0002$

Resultados

Função Objetivo $=0.718$

iterações $=10$

tempo $=0.125$ 
IEEE30 - Esquema L-E

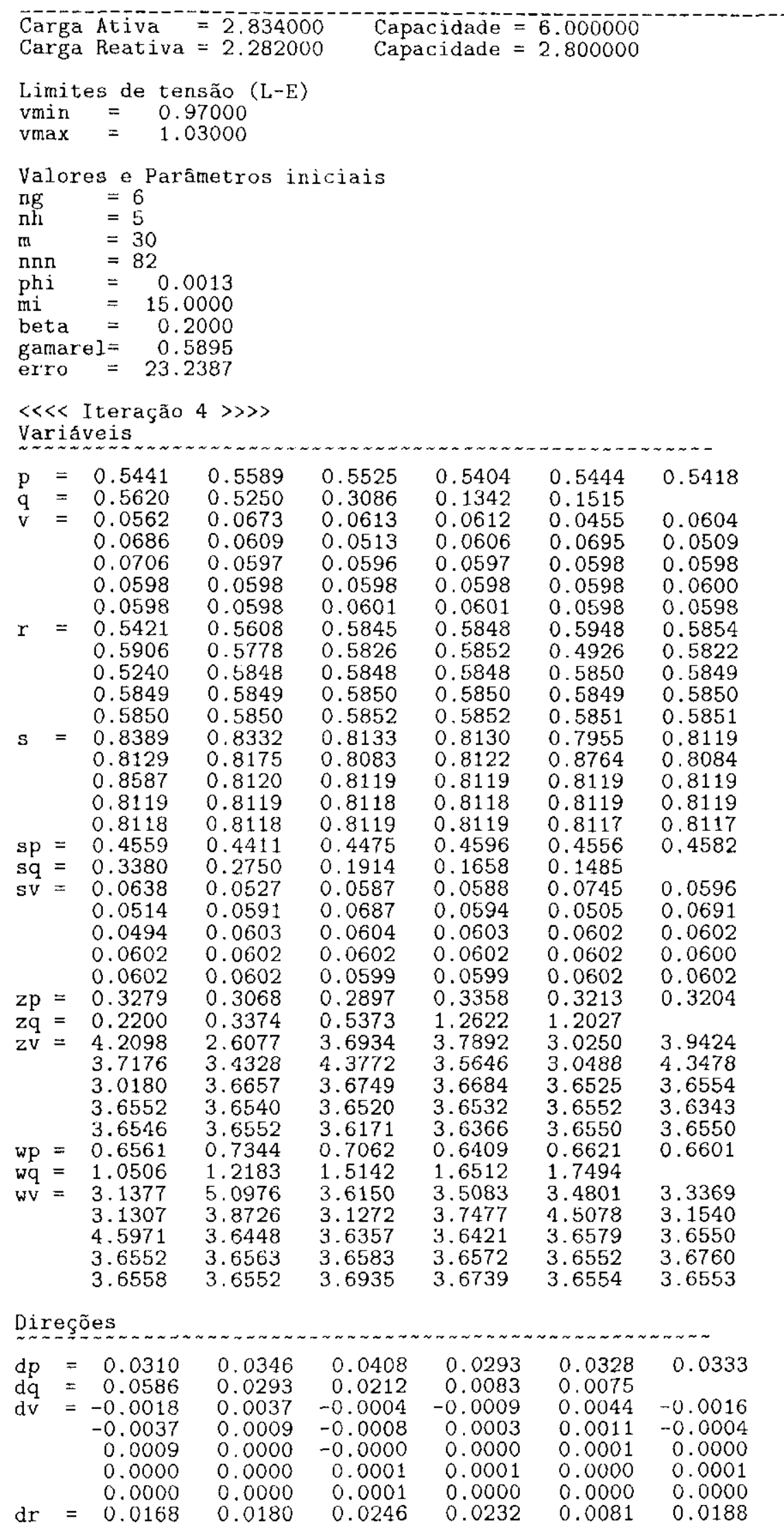




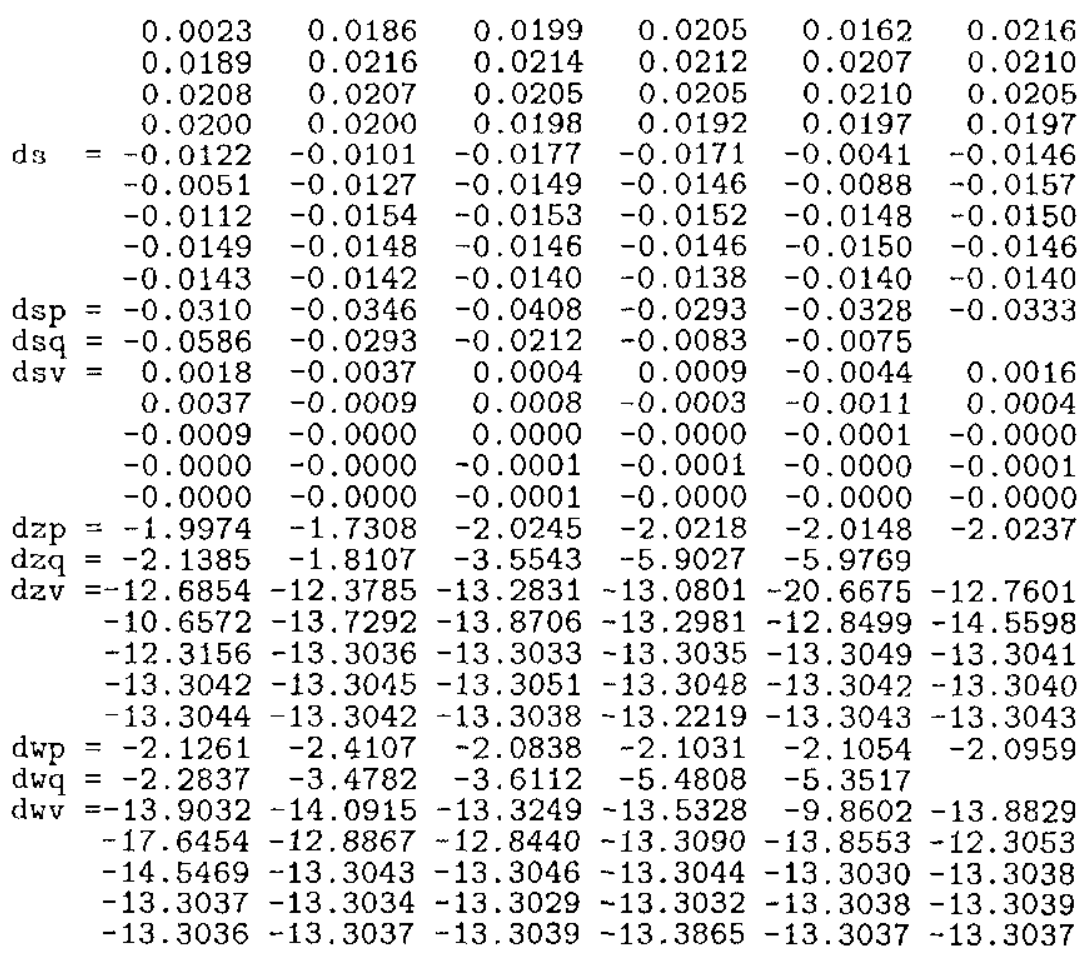
Residuos

\begin{tabular}{|c|c|c|c|c|c|c|c|}
\hline \multirow[t]{5}{*}{ rp } & $=$ & 0.0010 & -0.0011 & 0.2345 & 0.0751 & -0.0003 & 0.3843 \\
\hline & & & & & & & \\
\hline & & & -0. & & & & \\
\hline & & -0. & -0 . & & & -0 & \\
\hline & & & -0 & & & & \\
\hline \multirow[t]{5}{*}{$\mathrm{rq}$} & & 0.3 & & -0 . & & & \\
\hline & & -0.1 & & & & & \\
\hline & & & -0. & -0 . & -0 & -0. & \\
\hline & & & -0 . & -0 . & & -0.0 & \\
\hline & & & -0.0 & & & & \\
\hline \multirow[t]{4}{*}{$r v$} & & -0.0 & -0.0 & -0.0 & -0 . & & \\
\hline & & & & & & & \\
\hline & & & & & & & \\
\hline & & & & & & & \\
\hline & $=$ & -0.0 & & -0. & & & \\
\hline & & & & & & & \\
\hline & & & & & & & \\
\hline & & & & & -0 & & \\
\hline & & & & & & & \\
\hline \multirow[t]{5}{*}{ rs } & $=$ & -0 . & & -0 . & & & \\
\hline & & & & & & & \\
\hline & & & & & & & \\
\hline & & & & & & & \\
\hline & & & & & & & \\
\hline & $=$ & & & & & & \\
\hline $\mathrm{rsq}$ & $=$ & & & & & & \\
\hline \multirow[t]{5}{*}{ rs } & & -0 . & & & & & \\
\hline & & & & & & & \\
\hline & & & & & & & \\
\hline & & & & & & & \\
\hline & & & & & & & \\
\hline & $=$ & & -0.8 & -0. & & & -0 \\
\hline & & & & & & & \\
\hline & & & & & -0 . & -0.7 & -0.815 \\
\hline & & & & & & & \\
\hline & & & $\begin{array}{l}-0.7 \\
-0.7\end{array}$ & & & $\begin{array}{l}-0.7 \\
-0.7\end{array}$ & $\begin{array}{l}-0 . \\
-0\end{array}$ \\
\hline & & & -0.75 & -0.7 & -0.7 & -0.7 & \\
\hline & & -1.1116 & -1.2433 & -1.1187 & -1.0996 & -1.1091 & -1.1117 \\
\hline
\end{tabular}




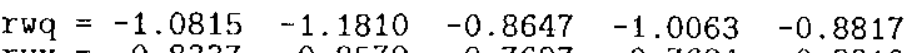

WV $=\begin{array}{llllll}-0.8337 & -0.8579 & -0.7697 & -0.7684 & -0.8310 & -0.7802\end{array}$ $\begin{array}{llllll}-0.7737 & -0.7885 & -0.8608 & -0.7984 & -0.7340 & -0.8401\end{array}$ $\begin{array}{llllll}-0.746 & -0.8028 & -0.8031 & -0.8029 & -0.8025 & -0.8026\end{array}$ $\begin{array}{llllll}-0.8026 & -0.8025 & -0.8025 & -0.8025 & -0.8026 & -0.8017 \\ -0.8025 & -0.8026 & -0.8009 & -0.8021 & -0.8025 & -0.8026\end{array}$

$\underset{\sim}{\text { Normas }}$

norm $(\mathrm{rp}) / \mathrm{np}=0.0961$

$\operatorname{norm}(\mathrm{rq}) / \mathrm{nq}=0.0654$

norm $(\mathrm{rv}) / \mathrm{nv}=0.0005$

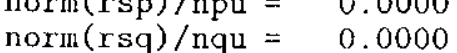

$\begin{array}{ll}\operatorname{norm}(\mathrm{rsq}) / \mathrm{nqu}= & =0.0000 \\ \operatorname{norm}(\mathrm{rsv}) / \mathrm{nvu}= & 0.0000\end{array}$

$\begin{array}{ll}\operatorname{norm}(\mathrm{rsv}) / \mathrm{nvu} & =0.0000 \\ \operatorname{norm}(\mathrm{rr}) / \mathrm{nc} & =0.0456\end{array}$

$\begin{array}{ll}\operatorname{norm}(\mathrm{rr}) / \mathrm{nc} & =0.0456 \\ \operatorname{norm}(\mathrm{rs}) / \mathrm{nc} & =0.0254\end{array}$

$\underset{\sim \sim \sim}{\text { Varáveis }} \underset{\sim \sim \sim}{\operatorname{Livres}}$

$\begin{array}{lllllll}\mathrm{yp}= & 0.8723 & 0.9866 & 0.9691 & 0.8455 & 0.8851 & 0.8816\end{array}$

$\mathrm{yq}=\begin{array}{rrrrr}0.8307 & 0.8809 & 0.9769 & 0.3889 & 0.5467\end{array}$

$\begin{array}{rrrrr}-1.0721 & 2.4899 & -0.0784 & -0.2809 & 0.4551 \\ -0.6054 & -0.5869 & 0.4398 & -1.2500 & 0.1832\end{array}$

$\begin{array}{rrrrr}1.4590 & -1.1938 & 1.5791 & -0.0210 & -0.0393\end{array}$

$\begin{array}{lllll}-0.0263 & 0.0054 & -0.0004 & 0.0000 & 0.0023 \\ 0.0063 & 0.0041 & -0.0001 & 0.0417 & 0.0013\end{array}$

$\begin{array}{lllll}0.0063 & 0.0041 & -0.0001 & 0.0417 & 0.0013\end{array}$

Parâmetros

$\mathrm{mi}=0.1600$

beta $=0.2000$

gamarel $=0.079$

Resultados

Função Objetivo $=0.898$

iterações $=4$

$\begin{array}{ll}\text { tempo } & =0.047 \\ \text { flops } & =98435\end{array}$ 